\title{
Cape Verde : Third Review Under the Policy Support Instrument—Staff Report; Staff Supplement; Press Release on the Executive Board Discussion; and Statement by the Executive Director for Cape Verde
}

In the context of the third review under the Policy Support Instrument for Cape Verde, the following documents have been released and are included in this package:

- $\quad$ The staff report for the Third Review Under the Policy Support Instrument, prepared by a staff team of the IMF, following discussions that ended on October 15, 2007 with the officials of Cape Verde on economic developments and policies. Based on information available at the time of these discussions, the staff report was completed on November 29, 2007. The views expressed in the staff report are those of the staff team and do not necessarily reflect the views of the Executive Board of the IMF.

- $\quad$ A staff supplement on the joint IMF/World Bank debt sustainability analysis.

- $\quad$ A Press Release summarizing the views of the Executive Board as expressed during its December 21, 2007 discussion of the staff report that completed the review.

- $\quad$ A statement by the Executive Director for Cape Verde.

The documents listed below have been or will be separately released.

Letter of Intent sent to the IMF by the authorities of Cape Verde*

Memorandum of Economic and Financial Policies by the authorities of Cape Verde*

Technical Memorandum of Understanding

*Also included in Staff Report

The policy of publication of staff reports and other documents allows for the deletion of market-sensitive information.

To assist the IMF in evaluating the publication policy, reader comments are invited and may be sent by e-mail to publicationpolicy@imf.org.

Copies of this report are available to the public from

International Monetary Fund $\bullet$ Publication Services

$70019^{\text {th }}$ Street, N.W. • Washington, D.C. 20431

Telephone: (202) 623-7430 • Telefax: (202) 623-7201

E-mail: publications@imf.org • Internet: http://www.imf.org

Price: $\$ 18.00$ a copy

International Monetary Fund

Washington, D.C. 

INTERNATIONAL MONETARY FUND

CAPE VERDE

\section{Third Review Under the Policy Support Instrument}

Prepared by the African Department

(In consultation with other departments)

Approved by David Andrews and Mark Plant

November 29, 2007

- Main topics: The report recommends waivers for the nonobservance of two assessment criteria and completion of the third review under the Policy Support Instrument (PSI) and summarizes the main macroeconomic and structural reform policies for 2008 .

- PSI: The Board completed the second PSI review on May 7, 2007. Program objectives include reducing public debt, building up international reserves, improving public financial management, and strengthening financial sector and energy sector regulation.

- Discussions: The mission met with Cristina Duarte, Minister of Finance and Public Administration; Carlos A. de Burgo, Governor of the Bank of Cape Verde; other officials; and representatives of commercial banks and the energy sector. The mission team comprised Mr. MacFarlan (outgoing mission chief), Mr. Leigh (incoming mission chief), Mr. Maehle, Mr. Castro, and Mr. Shanghavi (all AFR). Mr. Sembene of the Executive Director's Office and Ms. Francisco of the World Bank accompanied the mission.

- Exchange rate regime: Cape Verde's de facto and de jure exchange rate arrangement is a conventional peg fixed to the euro. 


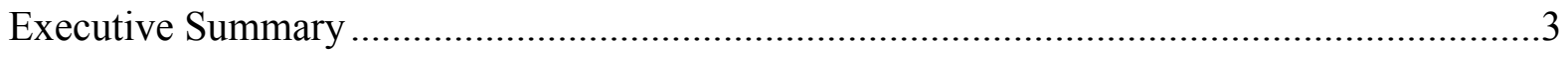

I. Strong Economic and Policy Performance but Challenges Remain...................................4

II. Current Economic Setting and Outlook and Performance Under the PSI .........................4

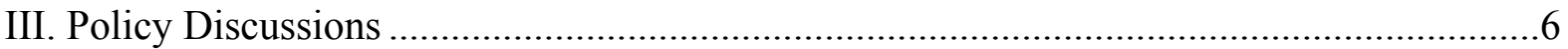

A. Fiscal Policy: What's Next as Debt Reduction is Running Ahead of Schedule? .....6

B. Exchange Rate, Monetary Management and Financial Sector Reform:

Safeguarding Financial Stability ..................................................................... 10

C. PFM Reforms: Enhanced Budget Execution and Monitoring ..............................12

D. Energy Sector Reforms: Reducing Fiscal Risk and Bolstering Growth ................13

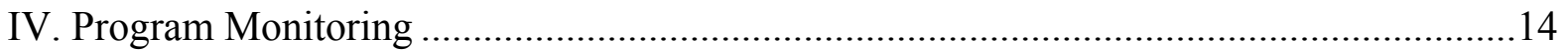

V. Staff Appraisal ................................................................................................. 14

Figures

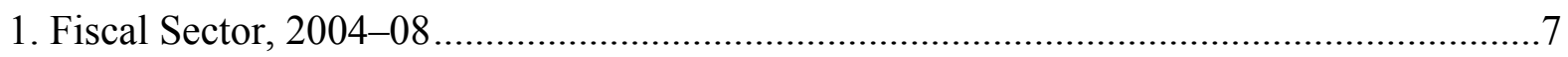

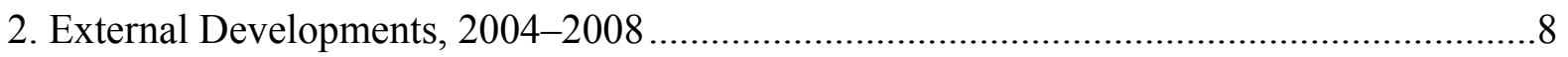

3. Monetary Developments, January 2004-September 2007 ..........................................9

Tables

1. Selected Economic and Financial Indicators, 2005-10 ..............................................16

2. Annual Fiscal Operations of the Central Government (CVEsc), 2006-10........................17

3. Annual Fiscal Operations of the Central Government (percent of GDP), 2006-10 ...........18

4. Balance of Payments, 2005-10 ............................................................................... 19

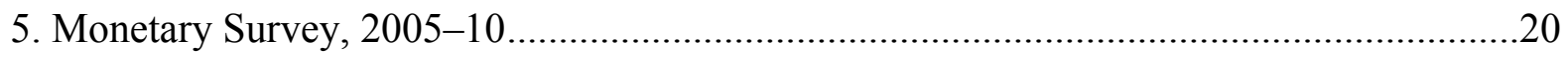

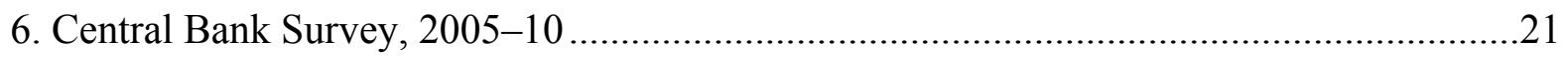

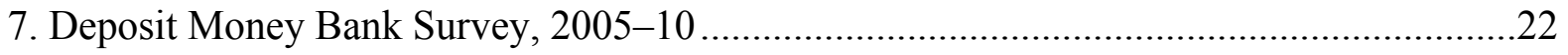

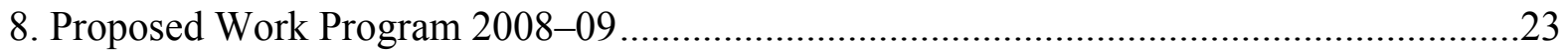

Appendices

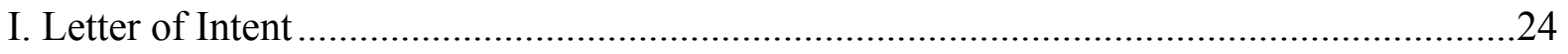

Attachment I: Memorandum of Economic and Financial Policies ..............................25

Attachment II: Technical Memorandum of Understanding .......................................35 


\section{EXECUTIVE SUMMARY}

\section{Cape Verde's strong performance continues under the PSI program and staff recommends completion of the third review.}

- $\quad$ GDP growth remains strong, unemployment and poverty rates are falling, inflation pressures remain contained and all quantitative assessment criteria for end-June 2007 were met comfortably. The robust growth is expected to continue over the medium term driven by the tourism boom and related foreign direct investment (FDI) flows.

- $\quad$ Fiscal consolidation and the build-up of official reserves have proceeded faster than program expectations. The initial projections for both domestic debt and official reserves for 2009 are likely to be reached in 2008.

- $\quad H o w e v e r$, performance on structural reforms has been mixed and two assessment criteria were missed. The new mechanism for setting base utility tariffs was not implemented and the mechanisms for adjusting utility and fuel prices were not applied. On the basis of remedial measures taken by the authorities, staff recommends granting waivers for the two missed structural assessment criteria.

\section{Key Issues and Policy Discussions for the 2008 program under the PSI}

- $\quad$ Staff supports the main thrust of the authorities' 2008 budget which is based on conservative revenue forecasts and measured expenditure growth. Beyond 2008, staff notes that the authorities should consider further fiscal consolidation to ensure that domestic demand growth allows for the reserve buildup and thereby reduce vulnerabilities to shocks. The authorities broadly concurred, and also expressed their intention to consider using some of the fiscal space for modest tax cuts and to scale up capital spending to ease infrastructure bottlenecks.

- $\quad$ Progress is being made to address the long-standing weaknesses in public sector financial management to improve budget execution and monitoring.

- $\quad$ The authorities are committed to move more rapidly on a comprehensive energy sector reform to improve utility and petroleum pricing mechanisms and encourage investment in the sector to reduce fiscal risk and support growth.

\section{Uncertainties and risks remain}

More flexible factor and product markets would enhance the ability of the economy to adjust to shocks; the recent decline in the flow of emigrant deposits provides challenges for monetary management; and there is need for large-scale investment to enable energy sector and infrastructure growth to keep pace with economic development. 


\section{Strong Economic and Policy Performance but Challenges Remain}

1. Cape Verde is at an important point in its history. Since 2001 disciplined macroeconomic management, more recently under the Policy Support Instrument (PSI), has yielded significant fiscal consolidation and supported strong economic growth with generally low inflation. Reflecting this solid performance, Cape Verde will graduate from the UN least-developed-country (LDC) status in early 2008. ${ }^{1}$ While this graduation has benefits, it also brings challenges: Cape Verde will have to prepare to become less dependent on aid. Thus, the key goal of the PSI is to help the country prepare for the opportunities and challenges associated with its graduation from LDC status in 2008.

\section{To further the reform agenda, discussions during the third review of the PSI} focused on:

- Consolidating macroeconomic stability gains to support reserves buildup and reduce vulnerabilities to shocks including the expected decline in concessional external financing;

- Improving public sector financial management to enhance budget execution and monitoring;

- Accelerating energy sector reform to reduce fiscal risk and support growth; and

- $\quad$ Enhancing financial sector reform to safeguard stability and enhance growth prospects.

\section{Current Economic Setting And Outlook And Performance Under the PSI}

3. Real GDP growth reached nearly 11 percent in 2006, the unemployment rate has declined, and fiscal and external positions have strengthened significantly.

- Growth has improved. Official data shows real GDP growth for 2006 of nearly 11 percent, the highest in almost a decade. Growth was particularly strong in tourism, telecommunications, and construction. Robust growth of 7 to 8 percent should continue this year and over the medium term, driven by tourism-related FDI, public infrastructure investment, and growth of financial services. Although still high at nearly 20 percent, the unemployment rate fell by 6 percentage points in 2006 .

\footnotetext{
${ }^{1}$ In late October, the European Commission formally recommended a strategic partnership with Cape Verde to the European Parliament and European Council bringing Cape Verde one step closer to special status with the European Union.
} 


\begin{tabular}{|c|c|c|c|c|c|}
\hline \multicolumn{6}{|c|}{$\begin{array}{c}\text { Cape Verde: Contribution to Growth } \\
\text { (Annual percentage change) }\end{array}$} \\
\hline & 2003 & 2004 & 2005 & 2006 & 2007 \\
\hline Agriculture and fishing & 0.1 & 0.0 & 0.0 & 0.1 & 0.0 \\
\hline Industry and energy & 0.3 & 0.3 & 0.7 & 0.5 & 0.3 \\
\hline Construction & 0.0 & 0.0 & 1.4 & 1.5 & 1.7 \\
\hline Commerce & 0.8 & 1.0 & 2.0 & 0.9 & 0.9 \\
\hline Hotels and restaurants & 0.7 & 0.5 & 0.7 & 1.5 & 0.9 \\
\hline Transport and communications & 0.6 & 1.0 & 0.2 & 3.3 & 1.4 \\
\hline Banks and insurance ${ }^{1}$ & -0.4 & -0.1 & -0.1 & 0.1 & 0.0 \\
\hline Public service & 0.8 & 1.2 & 0.9 & 1.2 & 0.6 \\
\hline Other $^{2}$ & 1.8 & 0.5 & 0.8 & 1.8 & 1.2 \\
\hline GDP & 4.7 & 4.3 & 6.5 & 10.8 & 6.9 \\
\hline
\end{tabular}

- Inflation pressures remain moderate. Consumer prices increased by 5 percent in the 12-months to October 2007 largely reflecting supply shocks (poor rainfall temporarily drove up domestically produced food prices). The 12-month inflation rate is projected to reach 53/4 percent by December 2007 (5 percent annual average) and to fall thereafter to 2-3 percent consistent with the peg and inflation in the euro area. ${ }^{2}$

- Domestic debt reduction is running ahead of program schedule (Figure 1). Tax revenues to date have exceeded budget forecasts, reflecting economic growth and improvements in tax administration, and asset sales

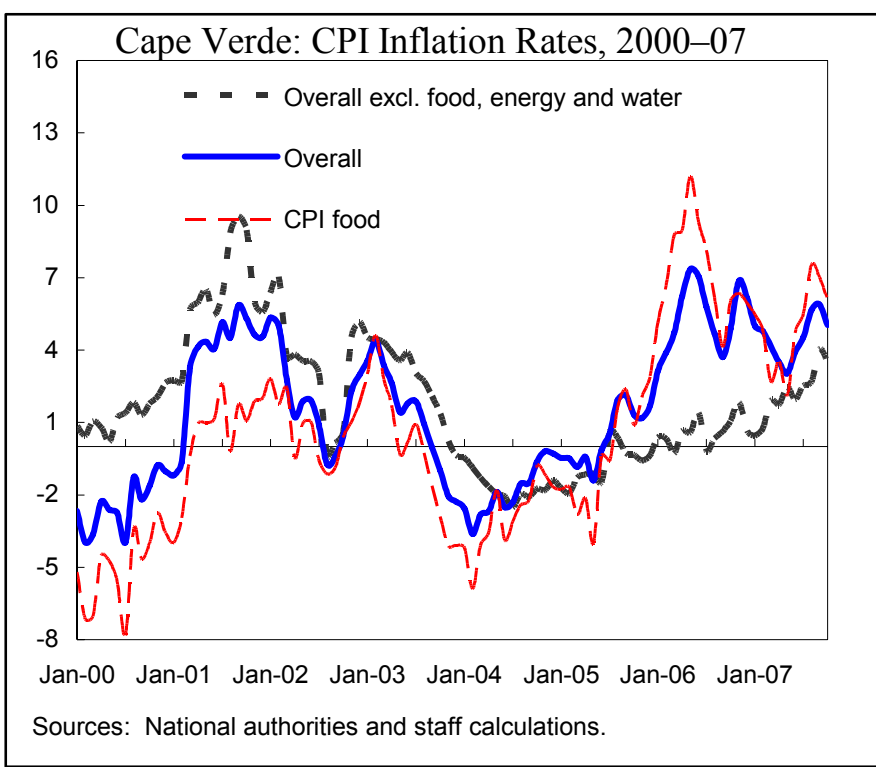
have surpassed expectations. These factors, together with expenditure restraint, are expected to low0065r the domestic debt-to-GDP ratio below the initial PSI target.

- $\quad$ Reserve accumulation through June 2007 exceeded the program target and rapid accumulation has continued. Reserves at end-September reached 33/4 months

\footnotetext{
${ }^{2}$ These projections are based on current CPI. A new CPI is expected to be released by end November with data revised back to January 2006. The new index is expected to be less susceptible to large swings in prices of a few domestically produced food items.
} 
of prospective imports and 130 percent of base money (Figure 2). Part of the accumulation in the first half of 2007 reflects banks drawing down on their net foreign assets position to finance purchase of central bank of Cape Verde (BCV) bills and investments in stocks and bonds on the Stock Exchange. While increasing FDIrelated imports are expected to raise the current account deficit, there are no signs of a general increase in imports that would jeopardize reserves.

\section{Within the framework of the exchange rate peg, $\mathrm{BCV}$ continues to manage} liquidity, and interest rate differentials with the euro area. Its increased sterilization efforts, new investment opportunities for commercial banks on the Stock Exchange, and continued rapid growth in private sector credit have eased excess liquidity, causing domestic rates to rise in recent months. At the same time, reduced credit to government has dampened broad money growth (Figure 3).

5. However, performance on the structural reforms has been mixed, and two assessment criteria were missed. While the authorities have prevented accumulation of central government arrears, weaknesses in public finance management (PFM) still pose concerns. The new mechanism for setting base utility tariffs was not implemented and the mechanisms for adjusting electricity, water, and fuel prices were not fully and continuously applied during most of 2007. Moreover, three of the four structural benchmarks for end-June 2007, which include the implementation of the medium-term expenditure framework and the recommendations of the task force on financial sector reform were only partially met.

\section{Policy Discussions}

In the attached LOI and MEFP, the authorities reaffirm their commitment to the macroeconomic and structural reform objectives of the PSI program, anchored on the exchange rate peg to the euro. With program objectives unchanged since the PSI began and the authorities overperforming on key macroeconomic targets, policy discussions focused on consolidating fiscal and monetary management and accelerating structural reforms.

\section{A. Fiscal Policy: What's Next as Debt Reduction is Running Ahead of Schedule?}

6. The mission supported the main thrust of the authorities' 2008 budget (MEFP, para. 8) which is appropriately based on conservative revenue forecasts and measured expenditure growth. ${ }^{3}$ The implied fiscal balance allows for further reduction in domestic

\footnotetext{
${ }^{3}$ The budget was presented to Parliament on October $20^{\text {th }}$.
} 
Figure 1. Cape Verde: Fiscal Sector, 2004 - 08 (Percent of GDP)

Fiscal consolidation continues...

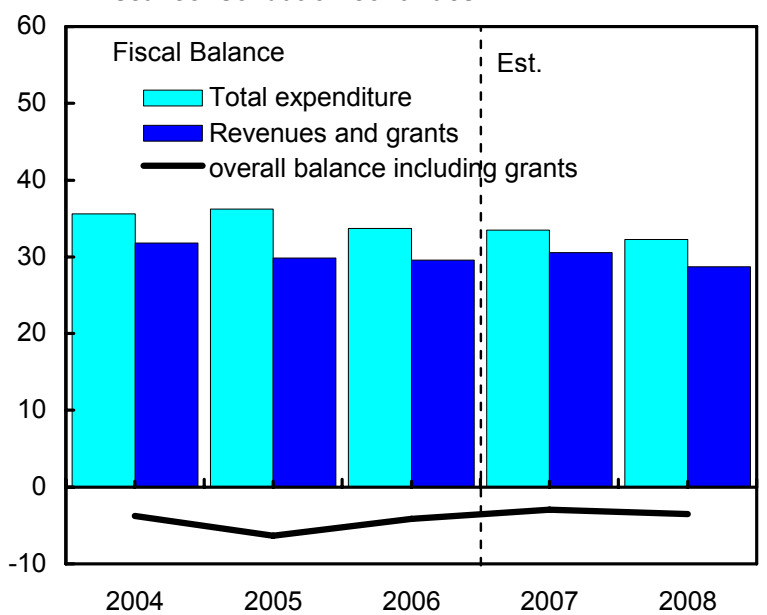

... and continued expenditure restraint...

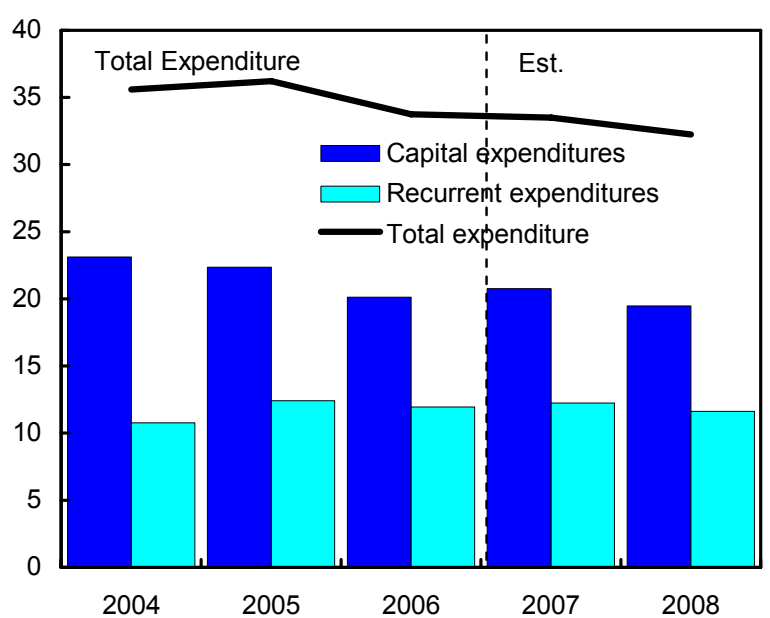

... driven by robust revenue performance...

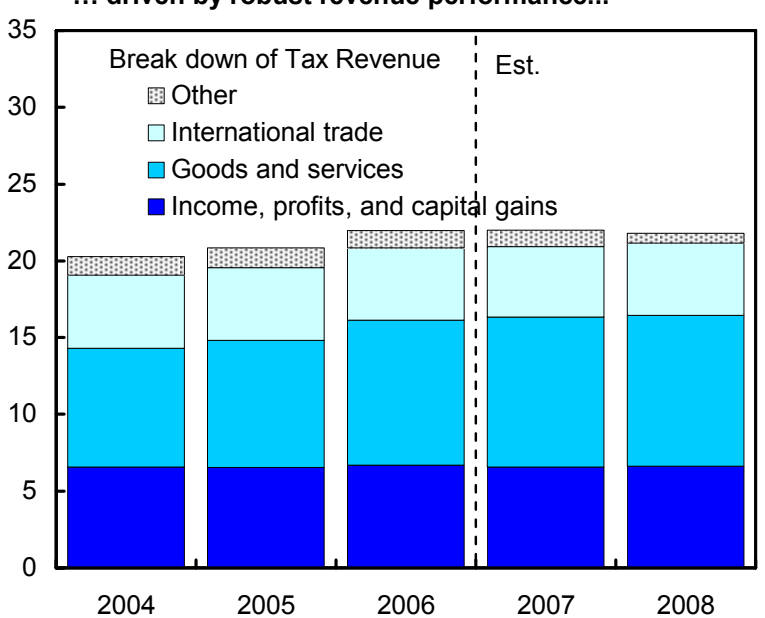

... thus debt reduction is running ahead of PSI target.

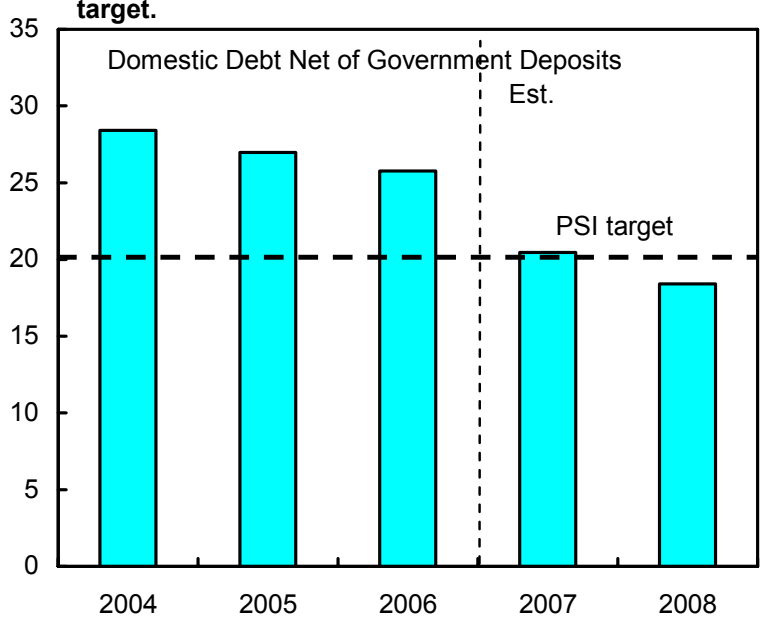

Source: National authorities and staff estimates. 
Figure 2. Cape Verde: External Developments, 2004 - 2008
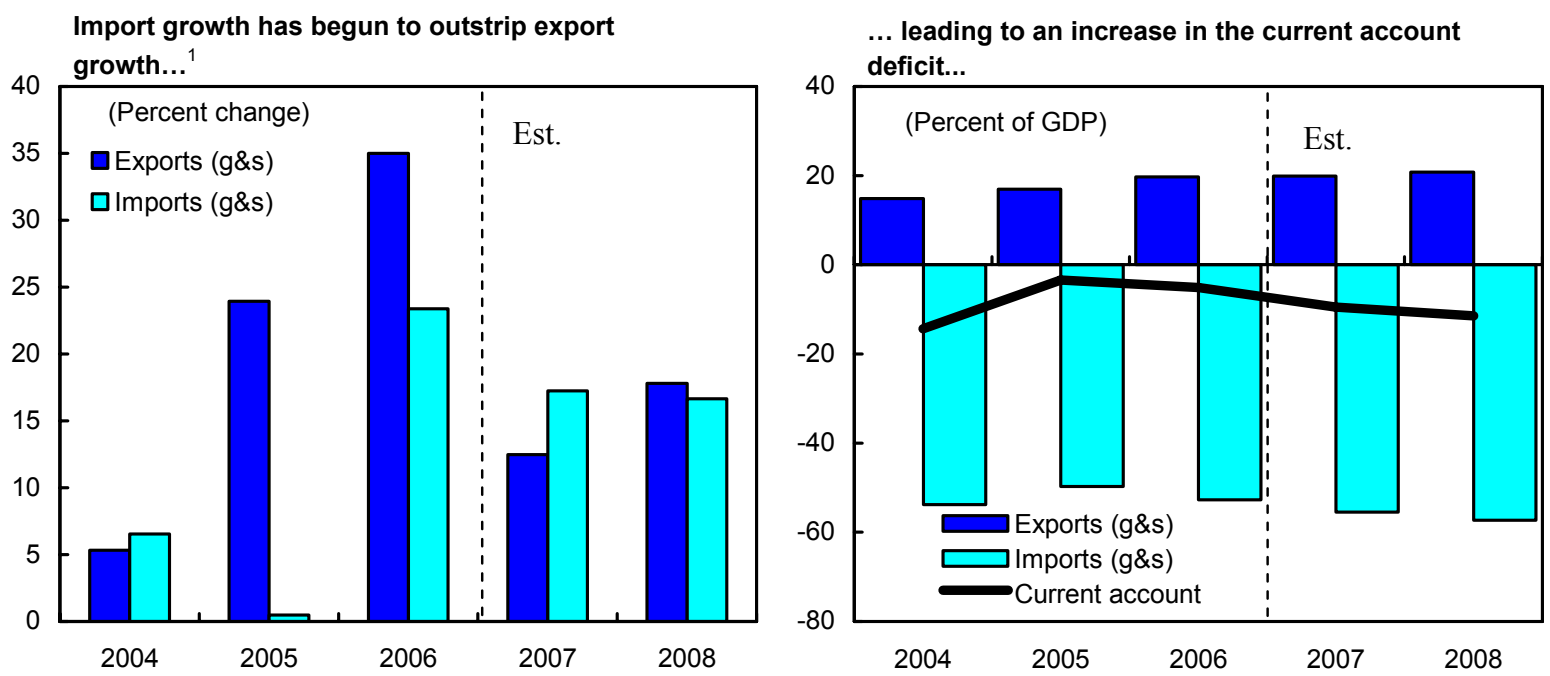

... which is more than financed by tourism related FDI and other financial flows...

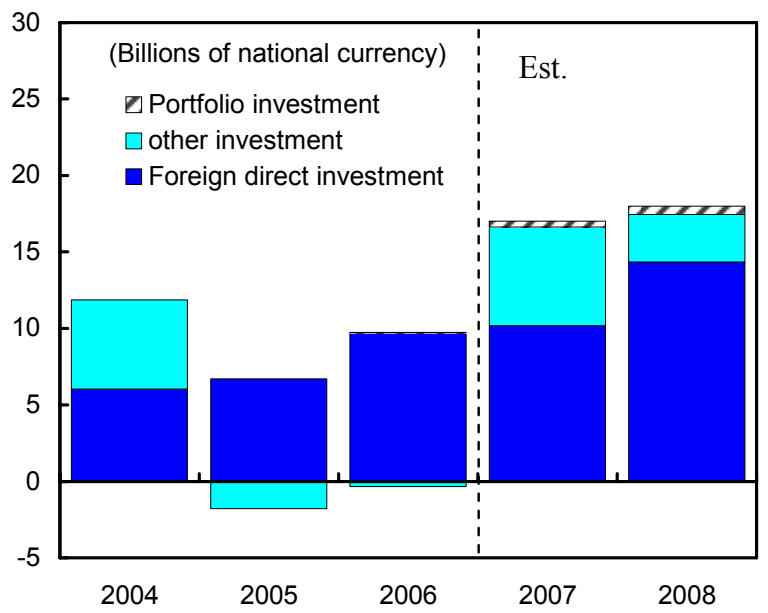

... leading to a rapid increase in gross international reserves.

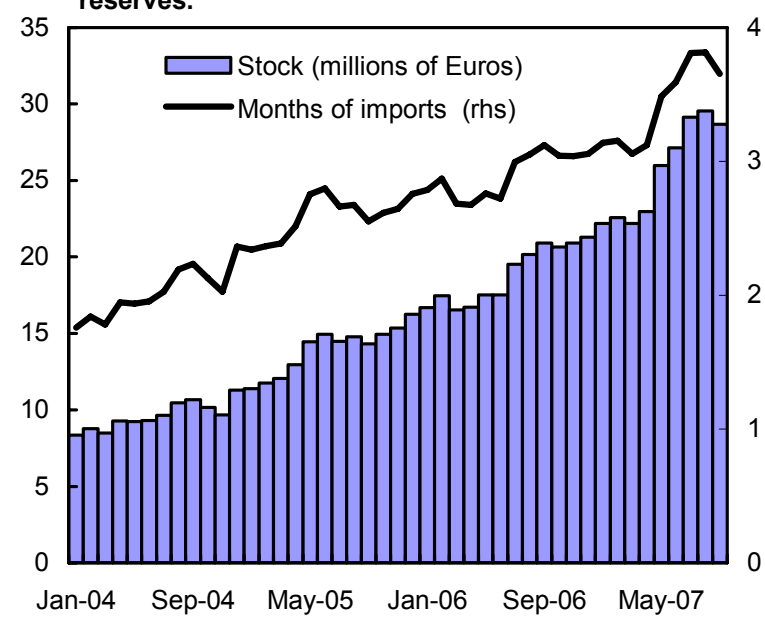

Source: National authorities and staff estimates.

1. Growth in total exports in 2007 was dampened by a sharp decline in reexports of fuel due to the stoppage of South African Airlines refueling in Cape Verde. 
Figure 3. Cape Verde: Monetary Developments, January 2004 - September 2007

Private sector credit growth is increasing and...

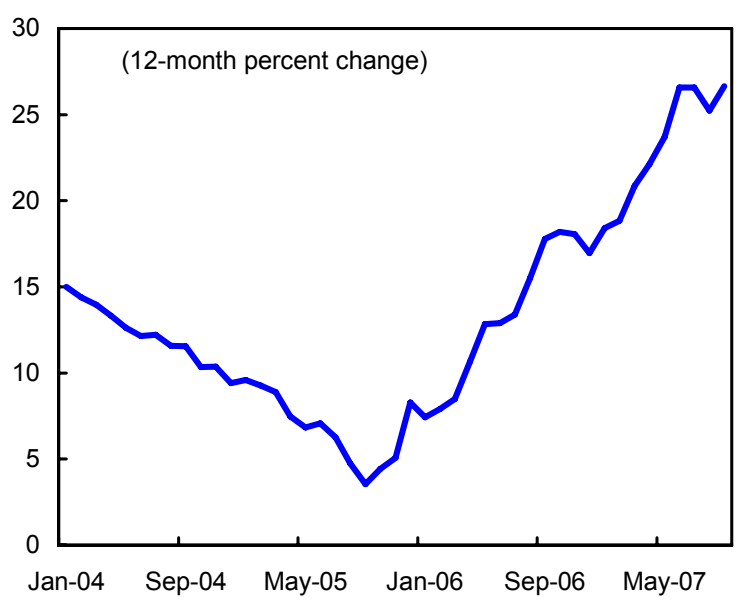

... as a result, excess liquidity is coming down, interest rates are edging up,..

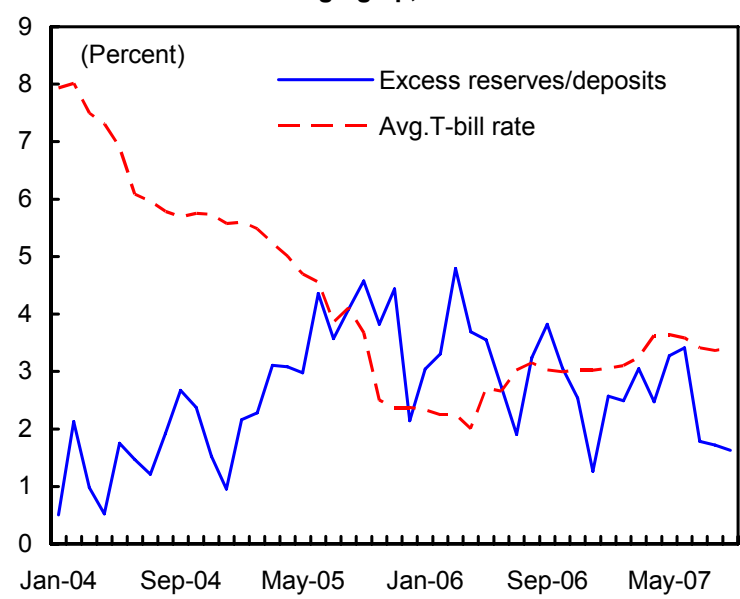

Broad money growth is tapering off...

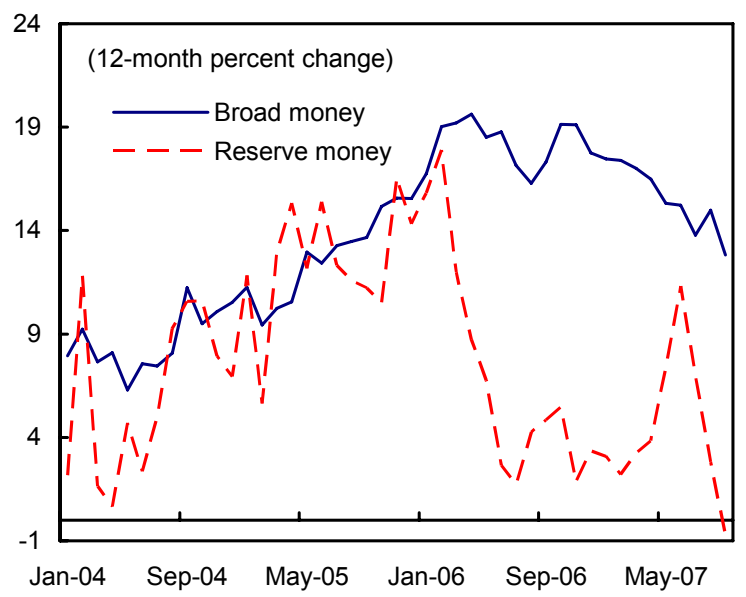

... the BCV intensifies its open market operations,...

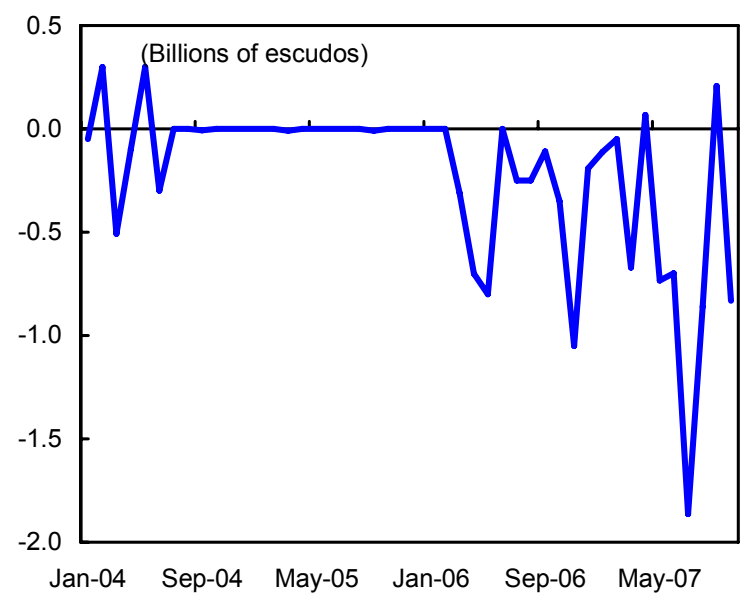

.... and commercial banks are unwinding their NFA.

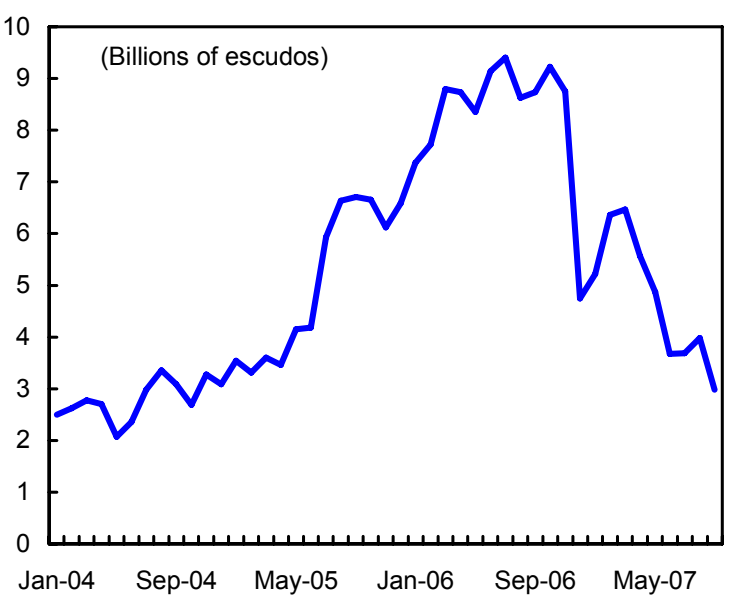

...as government bank debt declines, notwithstanding the growing money multiplier.

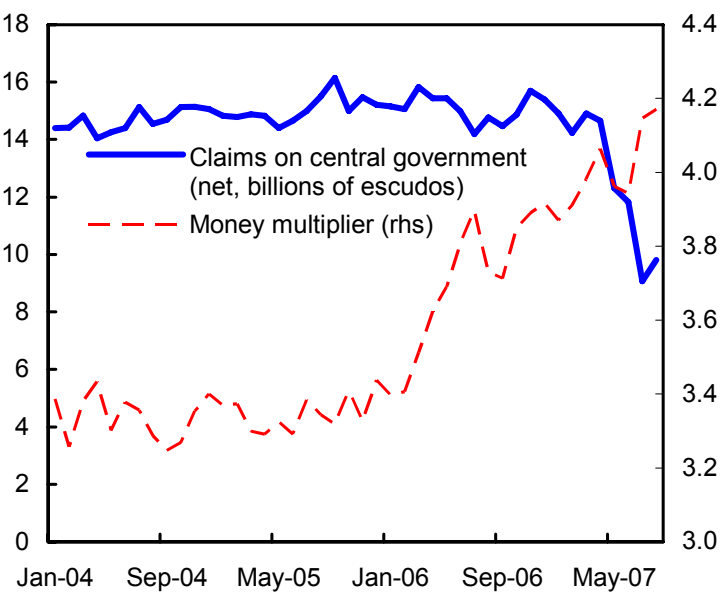

Source: National authorities and staff estimates. 
debt as a percent of GDP. Recurrent spending is contained to free resources for capital expenditures, and the budget allows for no utility and energy subsidies. Net domestic debt-toGDP ratio is projected to decline to below 20 percent by end-2008, a year ahead of the PSI schedule. Staff suggested that beyond 2008 the authorities consider further fiscal consolidation to ensure that domestic demand growth allows for further reserve buildup and thereby reduce vulnerability to shocks. Staff noted that the joint World Bank- IMF debt sustainability analysis shows that the most important risk to debt distress would arise from a return to imprudent fiscal policy, leading to a sharp rise in the debt burden.

\section{The authorities affirmed that preserving debt sustainability is a cornerstone of} their fiscal policy. They expressed their intention to consider, once the program debt target is achieved, some tax cuts such as reducing the corporate income tax rate from 30 to 25 percent to keep Cape Verde attractive to foreign investors and help seek public support for rationalizing tax exemptions and broadening the tax base. Once they finalize their mediumterm investment program, they also plan to use some of the expected fiscal space to scale up capital expenditure to ease infrastructure bottlenecks. ${ }^{4}$

\section{B. Exchange Rate, Monetary Management and Financial Sector Reform: Safeguarding Financial Stability}

\section{Over the last few years the exchange rate peg has served Cape Verde well as an anchor for financial stability, with both monetary management and fiscal policy} supporting the peg. The real effective exchange rate (REER) is broadly in line with fundamentals and policies as shown by staff's CGER-type analysis. Moreover, FDI and tourism exports are booming suggesting improved competitiveness; unemployment is declining; and reserves accumulating as targeted. The recent increase in the current account deficit is driven by FDI-related imports. With no natural resource advantage, Cape Verde's performance in attracting non-debt creating FDI flows relative to neighboring economies reflects the

Exchange Rate Developments, January 2000 - August 2007

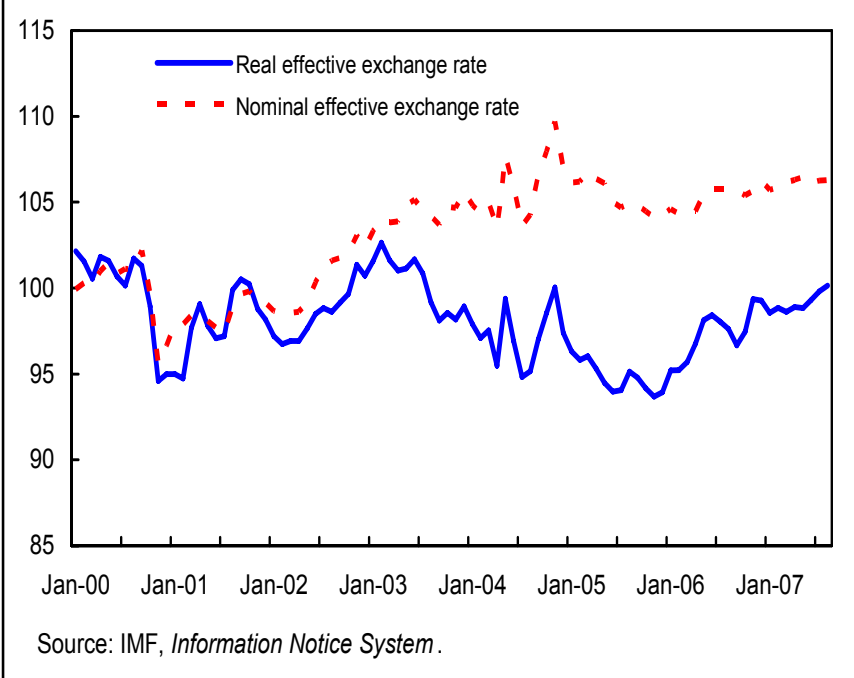
strength of its policies and reforms. The DSA also concludes that the risk of a debt distress is low. Furthermore, while the REER has fluctuated within a 5 percent band around a constant

\footnotetext{
${ }^{4}$ A joint Bank-Fund debt sustainability analysis shows that a temporary scaling up of capital investment will not increase the risk of debt distress in Cape Verde (Supplement to the Staff Report).
} 
trend for the last several years largely reflecting supply-side-driven inflation differentials, the rate has appreciated in the last eighteen months reversing the depreciation during 2004-05. This said, the recent significant appreciation of the euro against the US dollar warrants close monitoring.

\section{Monetary management remains fully consistent with the goal of building} foreign reserves to support the exchange rate peg. Staff noted that the large emigrant deposits - 40 percent of total bank deposits - continue to pose challenges for monetary management especially as emigrant deposit growth slowed markedly in the first half of 2007. ${ }^{5}$ Thus, careful management of external interest differentials is warranted to guard against an outflow of emigrant deposits.

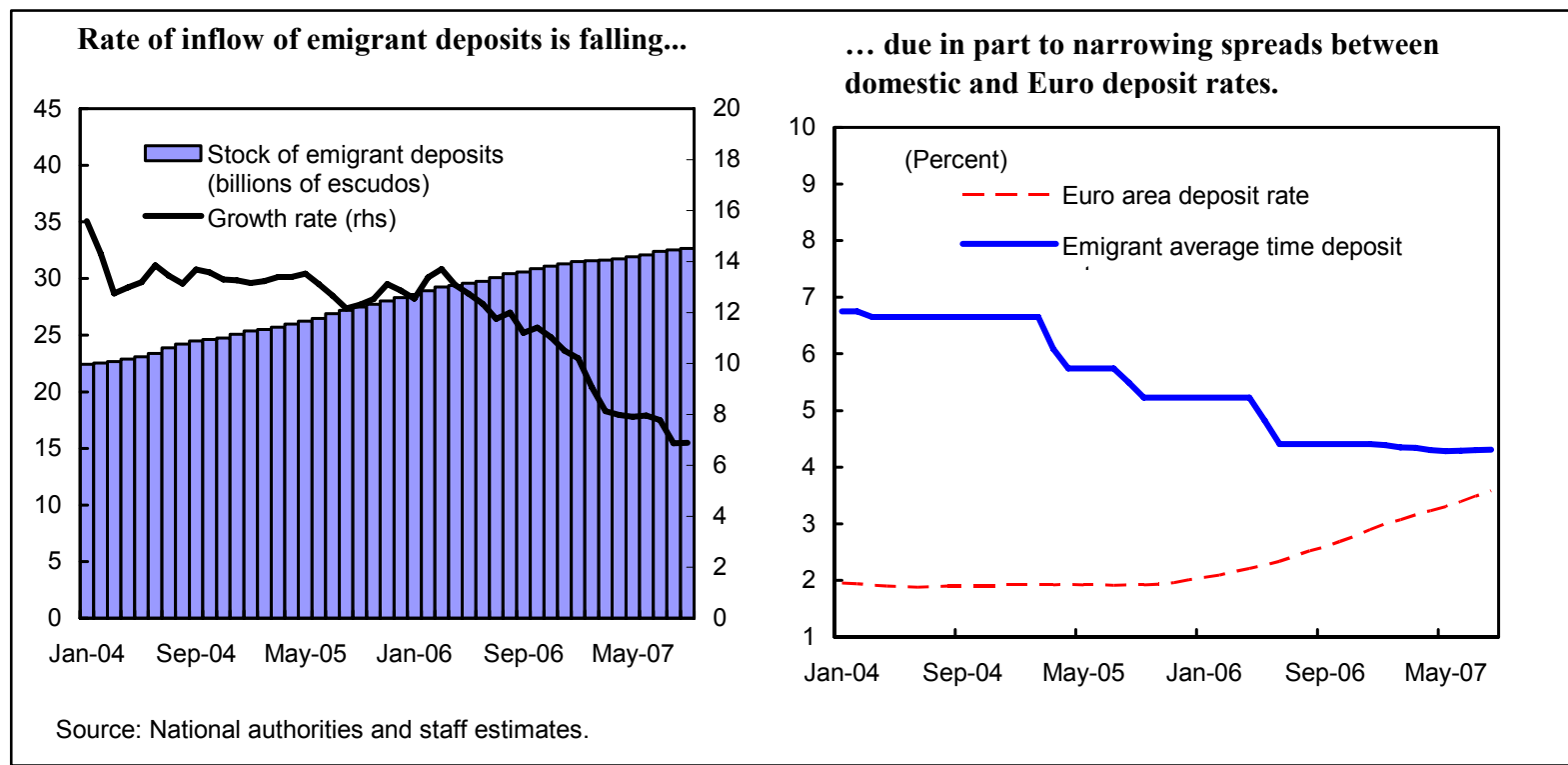

10. Staff urged the authorities to move rapidly to strengthen financial sector regulation and supervision. Abuse of Cape Verde's international financial center could cause significant reputational risks and third-party-enforcement actions, which could jeopardize growth and financial stability. Staff noted that strengthening the financial sector would be an important requirement for the authorities' planned liberalization of financial flows. Therefore, staff welcomed the measures committed to in their MEFP, including the three new benchmarks to establish a financial intelligence unit and strengthen the antimoney-laundering and combating-of-financing-of-terrorism framework.

\footnotetext{
${ }^{5}$ Total remittances from emigrants and overseas workers also declined markedly.
} 
11. The BCV and commercial banks concurred with staff's assessment of monetary management. They noted that the slowdown in emigrant deposits was due to new investment opportunities being created in Cape Verde and a substantial narrowing in recent years of the spread between emigrant and euro area deposit rates. The banks are responding to the slowdown by launching savings products for emigrants with significantly higher rates, and the BCV has pushed up domestic interest rates in line with the rise in European rates. ${ }^{6}$ The BCV will continue to monitor other indicators of monetary conditions and adjust policy operations as appropriate.

\section{The BCV emphasized their commitment to strengthen the regulation and} supervision of the financial sector (MEFP para. 17). Thus they reiterated their request for an early FSAP for Cape Verde. The staff broadly concurred, that their plan to further liberalize financial flows should not pose any near-term risk; it would largely validate the current de facto situation. Removal of the remaining restrictions, moreover, would facilitate financing of residents' participation in tourism projects and the further development of Cape Verde as an international financial center. ${ }^{7}$

\section{PFM Reforms: Enhanced Budget Execution and Monitoring}

13. Staff urged the authorities to strengthen budget execution and monitoring. PFM weaknesses have been a long-standing concern and contributed to repeated accumulation of arrears in the past, excess domestic borrowing, large statistical discrepancies in the fiscal accounts and weaknesses in fiscal data reporting. Staff also emphasized the need for strengthening the capacity to detect potential shortfalls in revenues and external budget support in time to take corrective actions. Thus staff recommended that the authorities introduce a mid-year review of fiscal developments to compare developments half way through the fiscal year with budget forecasts and allow corrective actions to be taken as necessary.

\section{The authorities responded that several initiatives are ongoing to strengthen} budget execution and monitoring (MEFP, para. 10). These reforms are shaped in part by the Country Financial Accountability Assessment action plan and World Bank Public Expenditure Reviews. They emphasized that it takes time for the benefit of these reforms to be fully realized and noted that capacity constraints have slowed the pace of PFM reform. Thus, the authorities are seeking additional technical assistance, including from the Fund to accelerate their PFM reforms.

\footnotetext{
${ }^{6}$ Banks continue to offer a 25-basis-point interest premium on some emigrant deposits, mainly for historical reasons, their size, and the additional foreign exchange business they bring.

${ }^{7}$ FDI transactions, portfolio investments, and forward foreign exchange transactions are liberalized. External borrowing and foreign currency deposit accounts are formally subject to BCV regulations, which currently are nonrestrictive.
} 


\section{Energy Sector Reforms: Reducing Fiscal Risk and Bolstering Growth}

\section{Staff reiterated that failure to fully and continuously apply the utility and} petroleum pricing mechanisms, the missed structural assessment criteria, represents fiscal risks and could pose a threat to economic growth and poverty reduction in Cape Verde. Utility and petroleum subsidies have for years been a serious drain on the budget: in 2006 accrued subsidies amounted to more than 2 percent of GDP. ${ }^{8}$ Utility tariffs below cost recovery and commercial losses have for years caused severe cash-flow problems for the electricity company, Electra. Thus, much-needed investments to improve Electra's performance - one of the main impediments to growth and poverty reduction in Cape Verde - has been held up, forcing large businesses to invest in their own electricity and water production facilities. Staff stressed that tariffs need to be kept in line with current costs to prevent a repeat of past Electra cash-flow problems. ${ }^{9}$ Automatic and transparent updates of utility tariffs and petroleum prices would help to depoliticize price setting, safeguard the budget and give companies incentives for investment and efficiency gains. Retail gasoline and diesel prices were increased by 15 and 11 percent respectively on October 24 which should clear the fiscal liabilities that had been built up since the last price adjustment in early 2007. Performance contracts with a new Electra management might induce managerial efficiency. Staff acknowledged however that it will take time before these measures result in sizable cost savings.

\section{The authorities laid out their medium-term strategy for a comprehensive} overhaul of the energy sector. They affirmed their commitment to the remedial measures for the two missed structural assessment criteria - the October fuel price adjustment, steps being taken to establish the base utility tariffs and strengthen the petroleum price adjustment mechanism (MEFP, para. 20-22). The government is also working to secure financing for Electra's investment program, including through the creation of a task force, and addressing the reasons for past underutilization of the program's non-concessional borrowing ceiling. Following the successful experience of collaborating with private firms in the utility sector on the island of Sal, this cooperation will be further encouraged to generate electricity to feed the Electra's distribution grid. In the oil sector, a new logistics company (to which the two importers will jointly import, store, and distribute petroleum) will allow economies of scale. A new and simplified fuel adjustment mechanism should help secure regular and transparent alignment of domestic fuel prices with international prices. In addition, Electra's management will be improved and generation and transmission capacity will be expanded, including with wind power.

\footnotetext{
${ }^{8}$ Part of this was paid in 2006. The rest was cleared in 2007.

${ }^{9}$ There should be no impact on the budget from the temporary delay in adjusting utility tariffs through endDecember 2007, and thereafter, utility prices would reflect market prices (para. 21 of the MEFP).
} 


\section{Program Monitoring}

17. Assessment criteria, benchmarks, and indicative targets for the next two reviews are in Tables 1 and 2 of the MEFP, including a proposed modification to the endDecember 2007 assessment criterion on net domestic borrowing set during the second review. The ceiling has been lowered to reflect the government's full unwinding of the higher than programmed domestic borrowing in 2006, and an adjuster has been added to safeguard against any unexpected delays in the proceeds from land sales (Appendix I, Attachment II, paragraph 2). The review schedule is in Table 8.

\section{Staff Appraisal}

18. Cape Verde's economic prospects continue to be favorable under the PSI program. Growth remains strong, unemployment and poverty rates are falling, inflation pressures remain contained and all quantitative assessment criteria for end-June 2007 were met comfortably. The peg continues to serve Cape Verde well and the staff believes that the real exchange rate is broadly in line with fundamentals. The authorities are to be commended for their prudent macroeconomic management; now they should move ahead to consolidate the gains made on macroeconomic stability. Important tasks also remain on the structural agenda which are critical to Cape Verde's growth.

\section{Further financial sector development would help to safeguard the sector's} stability and enhance growth prospects for Cape Verde. Abuse of Cape Verde's international financial center could give rise to significant reputational risks and third party enforcement actions, which could jeopardize growth and financial stability. The measures being taken by the government to strengthen financial sector regulation and supervision, including implementing the recommendations of the recent AML/CFT assessment by the Fund, are particularly important in this regard. Strengthening the financial sector would also provide the needed foundation for the authorities' plans to further liberalize financial flows.

20. Progress is being made to address the long-standing weaknesses in public sector financial management to improve budget execution and monitoring. The authorities have addressed the problem with accumulation of central government arrears. The measures outlined in the authorities' MEFP, including on the Integrated Online Budget Management System, should provide the government with better tools for budget execution and monitoring. A formal mid-year review of fiscal developments the authorities have committed to should help enhance budget monitoring and allow corrective actions to be taken if necessary.

21. The authorities should move rapidly to execute their plans on a comprehensive overhaul of the energy sector. The past failure to fully and continuously apply the utility and petroleum pricing mechanisms represents both fiscal risks and a threat to growth and poverty reduction. Automatic and transparent updates of utility tariffs and petroleum prices 
would depoliticize price setting, safeguard the budget, and give companies in the energy sector incentives for investment and efficiency gains.

22. Staff recommends completion of the third review of the PSI program. Based on the strength of the authorities' policies and the corrective measures taken, staff supports granting of waivers for the two structural assessment criteria that were missed. 
Table 1. Cape Verde: Selected Economic and Financial Indicators, 2005-10

\begin{tabular}{|c|c|c|c|c|c|c|}
\hline & 2005 & 2006 & 2007 & 2008 & 2009 & 2010 \\
\hline & & Prel. & \multicolumn{4}{|c|}{ Projections } \\
\hline & \multicolumn{5}{|c|}{ (Annual percentage change) } & \\
\hline \multicolumn{7}{|l|}{ National accounts and prices } \\
\hline Real GDP & 6.5 & 10.8 & 6.9 & 7.7 & 7.4 & 7.4 \\
\hline Real GDP per capita & 4.6 & 8.8 & 5.0 & 5.7 & 5.4 & 5.3 \\
\hline Consumer price index (annual average) & 0.4 & 5.4 & 4.9 & 4.0 & 1.7 & 2.0 \\
\hline Consumer price index (end of period) & 1.7 & 6.2 & 5.7 & 1.0 & 2.0 & 2.0 \\
\hline \multicolumn{7}{|l|}{ External sector } \\
\hline Exports of goods and services & 23.9 & 35.0 & 13.8 & 18.8 & 15.5 & 13.5 \\
\hline Of which: tourism & 17.8 & 82.5 & 37.5 & 30.4 & 22.8 & 16.9 \\
\hline Imports of goods and services & 0.5 & 23.4 & 18.1 & 16.9 & 16.4 & 14.6 \\
\hline Real effective exchange rate (annual average) & -2.3 & -0.2 & $\ldots$ & $\ldots$ & $\ldots$ & $\ldots$ \\
\hline Terms of trade (minus $=$ deterioration) & -5.2 & -5.1 & -0.3 & 1.1 & 1.8 & 1.6 \\
\hline \multicolumn{7}{|l|}{ Government finance } \\
\hline Total revenue (excluding grants) & 11.3 & 19.6 & 10.1 & 12.5 & 12.0 & 12.2 \\
\hline Total expenditure & 10.1 & 11.7 & 7.6 & 12.9 & 9.2 & 10.5 \\
\hline Noncapital expenditure & 3.7 & 11.5 & 7.2 & 3.5 & 12.0 & 5.4 \\
\hline Capital expenditure & 25.2 & 12.1 & 8.2 & 30.8 & 4.9 & 18.6 \\
\hline \multicolumn{7}{|l|}{ Money and credit } \\
\hline Net foreign assets & 58.8 & 14.8 & 25.6 & 20.6 & 16.7 & 12.4 \\
\hline Net domestic assets & 3.4 & 19.0 & 8.2 & 10.7 & 10.3 & 12.0 \\
\hline Of which: net claims on the central government & 0.9 & 1.2 & -38.7 & -7.5 & -9.9 & 1.9 \\
\hline credit to the economy ${ }^{1}$ & 9.0 & 17.3 & 27.1 & 13.8 & 13.2 & 13.2 \\
\hline Broad money (M2) & 15.5 & 17.7 & 13.3 & 13.9 & 12.5 & 12.1 \\
\hline Domestic broad money (M2X) & 17.3 & 22.4 & 13.3 & 13.9 & 12.5 & 12.1 \\
\hline \multirow[t]{2}{*}{ Income velocity (GDP/M2) } & 1.32 & 1.31 & 1.27 & 1.26 & 1.24 & 1.23 \\
\hline & \multicolumn{3}{|c|}{ (Percent of GDP) } & & & \\
\hline \multicolumn{7}{|l|}{ Saving-investment balance } \\
\hline Gross capital formation & 36.7 & 37.8 & 43.2 & 47.6 & 52.4 & 56.9 \\
\hline Government & 8.9 & 8.6 & 8.8 & 8.3 & 9.1 & 9.7 \\
\hline Nongovernment & 27.8 & 29.2 & 34.5 & 39.2 & 43.3 & 47.2 \\
\hline Gross national savings & 33.3 & 32.7 & 33.7 & 36.1 & 39.8 & 42.3 \\
\hline Of which: government & 6.2 & 9.5 & 7.4 & 6.6 & 7.8 & 8.0 \\
\hline External current account (including official current transfers) & -3.4 & -5.1 & -9.5 & -11.5 & -12.6 & -14.7 \\
\hline \multicolumn{7}{|l|}{ Government finance } \\
\hline Total revenue & 23.7 & 24.3 & 24.1 & 23.8 & 24.1 & 24.3 \\
\hline Total grants & 6.3 & 5.6 & 6.7 & 4.9 & 6.0 & 6.1 \\
\hline Total expenditure & 36.4 & 34.9 & 33.8 & 32.3 & 33.1 & 32.9 \\
\hline Overall balance before grants & -12.7 & -10.6 & -9.7 & -8.5 & -9.0 & -8.5 \\
\hline Overall balance (including grants) & -6.3 & -5.0 & -3.0 & -3.5 & -3.0 & -2.4 \\
\hline External financing (net) & 3.1 & 3.0 & 1.8 & 1.8 & 1.4 & 1.6 \\
\hline Domestic financing (net) & 0.8 & 2.9 & 0.9 & 1.7 & 1.6 & 0.9 \\
\hline Financing gap/ statistical discrepancy & 2.4 & -0.9 & 0.2 & 0.0 & 0.0 & 0.0 \\
\hline Total nominal government debt ${ }^{2}$ & 87.1 & 77.3 & 66.4 & 59.6 & 54.3 & 50.1 \\
\hline External government debt ${ }^{3}$ & 53.8 & 48.0 & 43.5 & 40.2 & 37.2 & 34.8 \\
\hline Domestic government debt, net of deposits & 33.3 & 29.3 & 22.9 & 19.4 & 17.1 & 15.3 \\
\hline External current account (excluding official current transfers) & -8.0 & -9.2 & -14.3 & -14.2 & -16.2 & -18.2 \\
\hline Overall balance of payments & 5.6 & 4.8 & 7.1 & 4.4 & 4.3 & 3.3 \\
\hline External current account (millions of euros, including official transfers) & -27.3 & -47.7 & -99.4 & -135.8 & -165.8 & -214.7 \\
\hline Gross international reserves (millions of euros, end of period) & 147.4 & 193.1 & 267.7 & 320.3 & 376.9 & 425.4 \\
\hline Gross international reserves to reserve money & 0.8 & 1.3 & 1.4 & 1.4 & 1.4 & 1.5 \\
\hline $\begin{array}{l}\text { Gross international reserves (months of prospective imports of goods } \\
\text { and services) }\end{array}$ & 2.8 & 3.1 & 3.6 & 3.7 & 3.8 & 3.9 \\
\hline External debt service (percent of exports of goods and services) & 8.6 & 5.7 & 5.0 & 4.5 & 4.3 & 3.8 \\
\hline \multicolumn{7}{|l|}{ Memorandum items: } \\
\hline Nominal GDP (billions of Cape Verde escudos) & 89.2 & 103.9 & 115.5 & 130.4 & 145.4 & 161.6 \\
\hline \multicolumn{7}{|l|}{ Exchange rate (Cape Verde escudos per U.S. dollar) } \\
\hline Period average & 88.7 & 87.8 & $\ldots$ & $\ldots$ & $\ldots$ & \\
\hline End period & 93.5 & 83.5 & $\ldots$ & $\ldots$ & $\ldots$ & \\
\hline
\end{tabular}

Sources: Cape Verdean authorities, and IMF staff estimates and projections.

' Excluding a December 2006 purchase of a Portuguese credit to Electra and subsequent offloading on the domestic securitity market.

${ }^{2}$ Net of central government deposits; including verified stock of domestic and external arrears.

${ }^{3}$ Excluding the claims on the offshore Trust Fund. 
Table 2. Cape Verde: Fiscal Operations of the Central Government, 2006-10

(Millions of Cape Verde escudos, unless otherwise indicated)

\begin{tabular}{|c|c|c|c|c|c|c|c|c|}
\hline & \multicolumn{2}{|c|}{2006} & \multicolumn{3}{|c|}{2007} & \multirow{2}{*}{$\frac{2008}{\text { Budget }}$} & \multirow{2}{*}{\multicolumn{2}{|c|}{$\begin{array}{ll}2009 \quad 2010 \\
\text { Proj. }\end{array}$}} \\
\hline & Program & Actual & Budget & Jan-Jun & Proj. & & & \\
\hline Revenue, grants, and net lending & 33,437 & 31,044 & 35,295 & 15,269 & 35,576 & 38,551 & 43,693 & 49,200 \\
\hline Domestic revenue (incl. net lending) & 24,604 & 25,255 & 26,963 & 14,203 & 27,812 & 31,286 & 35,039 & 39,324 \\
\hline Tax revenue & 21,099 & 22,828 & 24,178 & 12,916 & 25,454 & 28,059 & 32,140 & 36,074 \\
\hline Income and profit taxes & 6,955 & 6,952 & 7,497 & 3,930 & 7,580 & 8,462 & 9,749 & 10,939 \\
\hline Consumption taxes & 8,351 & 9,821 & 10,140 & 5,619 & 11,310 & 13,177 & 14,404 & 16,154 \\
\hline International trade taxes & 4,501 & 4,889 & 5,458 & 2,602 & 5,300 & 5,583 & 7,054 & 7,944 \\
\hline Other taxes & 1,291 & 1,166 & 1,083 & 765 & 1,265 & 837 & 933 & 1,037 \\
\hline Nontax revenue & 3,505 & 2,126 & 2,585 & 1,020 & 2,090 & 3,204 & 2,899 & 3,251 \\
\hline Net lending & 0 & 301 & 200 & 268 & 268 & 23 & 0 & 0 \\
\hline External grants & 8,833 & 5,789 & 8,332 & 1,066 & 7,764 & 7,265 & 8,654 & 9,875 \\
\hline Capital grants & 7,003 & 4,035 & 6,706 & 1,066 & 6,036 & 5,597 & 7,919 & 9,075 \\
\hline Budget support & 1,830 & 1,755 & 1,625 & 0 & 1,728 & 1,668 & 736 & 800 \\
\hline Total expenditure & 40,413 & 36,244 & 39,943 & 15,104 & 38,990 & 44,032 & 48,075 & 53,107 \\
\hline Recurrent expenditure & 22,416 & 18,887 & 22,564 & 10,786 & 24,014 & 24,950 & 28,320 & 31,237 \\
\hline Primary recurrent expenditure & 20,593 & 16,967 & 20,681 & 9,932 & 22,131 & 22,896 & 26,066 & 28,904 \\
\hline Wages and salaries & 12,712 & 11,547 & 13,165 & 5,946 & 13,165 & 14,245 & 16,504 & 18,433 \\
\hline Goods and services & 1,862 & 1,197 & 2,127 & 615 & 1,682 & 2,335 & 2,591 & 2,838 \\
\hline Transfers and subsidies & 5,146 & 3,464 & 3,869 & 2,971 & 5,764 & 4,453 & 4,935 & 5,405 \\
\hline Transfers & 3,921 & 3,172 & 3,834 & 1,627 & 3,927 & 4,395 & 4,871 & 5,333 \\
\hline Subsidies & 1,225 & 292 & 35 & 1,344 & 1,838 & 58 & 65 & 72 \\
\hline Of which: energy subsidies & 1,200 & 208 & 0 & 0 & $1,802^{5}$ & 0 & 0 & 0 \\
\hline Other expenditures & 873 & 760 & 1,520 & 400 & 1,520 & 1,863 & 2,035 & 2,228 \\
\hline Domestic interest payments & 1,300 & 1,398 & 1,352 & 597 & 1,352 & 1,487 & 1,567 & 1,580 \\
\hline External interest payments & 522 & 522 & 531 & 257 & 531 & 566 & 687 & 753 \\
\hline Extraordinary expenditures & 0 & 0 & 0 & 0 & 0 & 0 & 0 & 0 \\
\hline Capital expenditure & 16,290 & 12,415 & 16,340 & 3,534 & 13,438 & $17,581^{4}$ & 18,441 & 21,870 \\
\hline Foreign financed & 12,534 & 11,173 & 13,487 & 2,879 & 11,058 & 12,470 & 12,335 & 13,933 \\
\hline Domestically financed & 3,755 & 1,242 & 2,853 & 654 & 2,380 & 5,111 & 6,105 & 7,937 \\
\hline Other expenditures (incl. arrears clearance) & 1,708 & 4,942 & 1,039 & 784 & 1,537 & 1,500 & 1,314 & 0 \\
\hline Of which: energy subsidies & 0 & 204 & 0 & 0 & 0 & 0 & 0 & 0 \\
\hline Overall balance, including grants (budget basis) & $-6,976$ & $-5,200$ & $-4,648$ & 165 & $-3,414$ & $-5,481$ & $-4,381$ & $-3,907$ \\
\hline Financing & 6,976 & 5,200 & 4,648 & -165 & 3,414 & 5,481 & 4,381 & 3,907 \\
\hline Foreign (net) & 3,414 & 3,130 & 3,043 & 242 & 2,063 & 3,160 & 2,085 & 2,510 \\
\hline Domestic (net) & 3,575 & 3,019 & 1,605 & -671 & 1,087 & 2,321 & 2,297 & 1,397 \\
\hline Net domestic borrowing & 830 & 1,638 & -602 & $-1,865$ & $-1,201$ & -172 & 797 & -103 \\
\hline Banking system & 989 & 185 & $\ldots$ & $-3,563$ & $-5,959$ & 248 & -865 & 153 \\
\hline Nonbanks & -158 & 1,453 & $\ldots$ & 1,698 & $4,758^{6}$ & -421 & 1,661 & -256 \\
\hline Privatization and other sales of assets & 3,689 & 456 & 2,207 & 3,104 & 4,199 & 2,493 & 1,500 & 1,500 \\
\hline Accounts payable (atrasados), net & -944 & 925 & $\ldots$ & $-1,911$ & $-1,911$ & 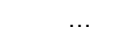 & 0 & 0 \\
\hline Net errors and omissions & 0 & -949 & 0 & 263 & 263 & 0 & 0 & 0 \\
\hline Financing gap & -13 & 0 & 0 & 0 & 0 & 0 & 0 & 0 \\
\hline \multicolumn{9}{|l|}{ Memorandum items: } \\
\hline $\begin{array}{l}\text { Overall balance, including grants (excluding } \\
\text { clearance of arrears and accounts payable) }\end{array}$ & $-4,324$ & $-4,113$ & $-3,609$ & 2,289 & -675 & $-3,980$ & $-3,068$ & $-3,907$ \\
\hline Arrears clearance & 1,708 & 2,012 & 1,039 & 213 & 828 & 1,500 & 1,314 & 0 \\
\hline Net domestic borrowing, excluding clearance of & & & & & & & & \\
\hline arrears and net accounts payable & $-1,821$ & 551 & $-1,641$ & $-3,988$ & $-3,940$ & $-1,673$ & -517 & -103 \\
\hline Primary balance (including grants) ${ }^{1}$ & $-5,154$ & $-3,280$ & $-2,765$ & 1,020 & $-1,531$ & $-3,427$ & $-2,127$ & $-1,574$ \\
\hline Recurrent domestic balance $^{2}$ & 2,188 & 6,368 & 4,400 & 3,417 & 3,798 & 6,335 & 6,719 & 8,087 \\
\hline Net external flows ${ }^{3}$ & 12,234 & 8,919 & 11,375 & 1,308 & 9,827 & 10,425 & 10,739 & 12,386 \\
\hline External debt service (percent of domestic revenue) & 10.7 & 9.9 & 9.8 & 7.8 & 9.5 & 8.3 & 8.3 & 7.5 \\
\hline Domestic debt (including arrears and accounts & & & & & & & & \\
\hline payable, net of deposits) & 27,902 & 30,397 & 25,426 & $\ldots$ & 26,457 & 24,785 & 24,817 & 24,713 \\
\hline
\end{tabular}

Sources: Ministry of Finance and Public Administration, Bank of Cape Verde, and IMF staff estimates and projections.

${ }^{1}$ Overall balance (including grants) - total expenditure + domestic and external interest payments.

${ }^{2}$ Domestic revenue - recurrent expenditure.

${ }^{3}$ External grants + net foreign financing.

${ }^{4}$ The capital expenditure budget is typically underexecuted.

${ }^{5}$ The energy subsidies recorded in 2007 were incurred in previous periods.

${ }^{6}$ Reflects borrowing from the pension fund. 
Table 3. Cape Verde: Fiscal Operations of the Central Government, 2006-10 (Percent of GDP)

\begin{tabular}{|c|c|c|c|c|c|c|c|c|}
\hline \multirow{3}{*}{ Revenue, grants, and net lending } & \multicolumn{2}{|c|}{2006} & \multicolumn{3}{|c|}{2007} & \multirow{2}{*}{$\frac{2008}{\text { Budget }}$} & \multicolumn{2}{|r|}{2010} \\
\hline & Program & Actual & Budget & Jan-Jun & Proj. & & \multicolumn{2}{|c|}{ Proj. } \\
\hline & 34.1 & 29.9 & 30.6 & 13.2 & 30.8 & 29.6 & 30.0 & 30.4 \\
\hline Domestic revenue (incl. net lending) & 25.1 & 24.3 & 23.3 & 12.3 & 24.1 & 24.0 & 24.1 & 24.3 \\
\hline Tax revenue & 21.5 & 22.0 & 20.9 & 11.2 & 22.0 & 21.5 & 22.1 & 22.3 \\
\hline Income and profit taxes & 7.1 & 6.7 & 6.5 & 3.4 & 6.6 & 6.5 & 6.7 & 6.8 \\
\hline Consumption taxes & 8.5 & 9.5 & 8.8 & 4.9 & 9.8 & 10.1 & 9.9 & 10.0 \\
\hline International trade taxes & 4.6 & 4.7 & 4.7 & 2.3 & 4.6 & 4.3 & 4.9 & 4.9 \\
\hline Other taxes & 1.3 & 1.1 & 0.9 & 0.7 & 1.1 & 0.6 & 0.6 & 0.6 \\
\hline Nontax revenue & 3.6 & 2.0 & 2.2 & 0.9 & 1.8 & 2.5 & 2.0 & 2.0 \\
\hline Net lending & 0.0 & 0.3 & 0.2 & 0.2 & 0.2 & 0.0 & 0.0 & 0.0 \\
\hline External grants & 9.0 & 5.6 & 7.2 & 0.9 & 6.7 & 5.6 & 6.0 & 6.1 \\
\hline Capital grants & 7.1 & 3.9 & 5.8 & 0.9 & 5.2 & 4.3 & 5.4 & 5.6 \\
\hline Budget support & 1.9 & 1.7 & 1.4 & 0.0 & 1.5 & 1.3 & 0.5 & 0.5 \\
\hline Total expenditure & 41.2 & 34.9 & 34.6 & 13.1 & 33.8 & 33.8 & 33.1 & 32.9 \\
\hline Recurrent expenditure & 22.8 & 18.2 & 19.5 & 9.3 & 20.8 & 19.1 & 19.5 & 19.3 \\
\hline Primary recurrent expenditure & 21.0 & 16.3 & 17.9 & 8.6 & 19.2 & 17.6 & 17.9 & 17.9 \\
\hline Wages and salaries & 13.0 & 11.1 & 11.4 & 5.1 & 11.4 & 10.9 & 11.4 & 11.4 \\
\hline Goods and services & 1.9 & 1.2 & 1.8 & 0.5 & 1.5 & 1.8 & 1.8 & 1.8 \\
\hline Transfers and subsidies & 5.2 & 3.3 & 3.3 & 2.6 & 5.0 & 3.4 & 3.4 & 3.3 \\
\hline Transfers & 4.0 & 3.1 & 3.3 & 1.4 & 3.4 & 3.4 & 3.3 & 3.3 \\
\hline Subsidies & 1.2 & 0.3 & 0.0 & 1.2 & 1.6 & 0.0 & 0.0 & 0.0 \\
\hline Of which: energy subsidies & 1.2 & 0.2 & 0.0 & 0.0 & $1.6^{\mathrm{b}}$ & 0.0 & 0.0 & 0.0 \\
\hline Other expenditures & 0.9 & 0.7 & 1.3 & 0.3 & 1.3 & 1.4 & 1.4 & 1.4 \\
\hline Domestic interest payments & 1.3 & 1.3 & 1.2 & 0.5 & 1.2 & 1.1 & 1.1 & 1.0 \\
\hline External interest payments & 0.5 & 0.5 & 0.5 & 0.2 & 0.5 & 0.4 & 0.5 & 0.5 \\
\hline Extraordinary expenditures & 0.0 & 0.0 & 0.0 & 0.0 & 0.0 & 0.0 & 0.0 & 0.0 \\
\hline Capital expenditure & 16.6 & 12.0 & 14.1 & 3.1 & 11.6 & $13.5^{4}$ & 12.7 & 13.5 \\
\hline Foreign financed & 12.8 & 10.8 & 11.7 & 2.5 & 9.6 & 9.6 & 8.5 & 8.6 \\
\hline Domestically financed & 3.8 & 1.2 & 2.5 & 0.6 & 2.1 & 3.9 & 4.2 & 4.9 \\
\hline Other expenditures (incl. arrears clearance) & 1.7 & 4.8 & 0.9 & 0.7 & 1.3 & 1.2 & 0.9 & 0.0 \\
\hline Of which: energy subsidies & 0.0 & 0.2 & 0.0 & 0.0 & 0.0 & 0.0 & 0.0 & 0.0 \\
\hline Overall balance, including grants (budget basis) & -7.1 & -5.0 & -4.0 & 0.1 & -3.0 & -4.2 & -3.0 & -2.4 \\
\hline Financing & 7.1 & 5.0 & 4.0 & -0.1 & 3.0 & 4.2 & 3.0 & 2.4 \\
\hline Foreign (net) & 3.5 & 3.0 & 2.6 & 0.2 & 1.8 & 2.4 & 1.4 & 1.6 \\
\hline Total drawings & 5.6 & 4.9 & 4.5 & 1.0 & 3.6 & 4.0 & 3.0 & 2.9 \\
\hline Amortization & -2.2 & -1.9 & -1.8 & -0.7 & -1.8 & -1.6 & -1.5 & -1.4 \\
\hline Domestic (net) & 3.6 & 2.9 & 1.4 & -0.6 & 0.9 & 1.8 & 1.6 & 0.9 \\
\hline Net domestic borrowing & 0.8 & 1.6 & -0.5 & -1.6 & -1.0 & -0.1 & 0.5 & -0.1 \\
\hline Banking system & $\ldots$ & $\ldots$ & $\ldots$ & -3.1 & -5.2 & 0.2 & -0.6 & 0.1 \\
\hline Nonbanks & $\ldots$ & $\ldots$ & $\ldots$ & 1.5 & $4.1^{6}$ & -0.3 & 1.1 & -0.2 \\
\hline Privatization and other sales of assets & 1.4 & 0.4 & 1.9 & 2.7 & 3.6 & 1.9 & 1.0 & 0.9 \\
\hline Accounts payable (atrasados), net & 2.3 & $\ldots$ & $\ldots$ & -1.7 & -1.7 & $\ldots$ & 0.0 & 0.0 \\
\hline Net errors and omissions & 0.0 & -0.9 & 0.0 & 0.2 & 0.2 & 0.0 & 0.0 & 0.0 \\
\hline Financing gap & 0.0 & 0.0 & 0.0 & 0.0 & 0.0 & 0.0 & 0.0 & 0.0 \\
\hline \multicolumn{9}{|l|}{ Memorandum items: } \\
\hline $\begin{array}{l}\text { Overall balance, including grants (excluding } \\
\text { clearance of arrears and accounts payable) }\end{array}$ & -4.4 & -4.0 & -3.1 & 2.0 & -0.6 & -3.1 & -2.1 & -2.4 \\
\hline Arrears clearance & 1.7 & 1.9 & 0.9 & 0.2 & 0.7 & 1.2 & 0.9 & 0.0 \\
\hline $\begin{array}{l}\text { Net domestic borrowing, excluding clearance of } \\
\text { arrears and net accounts payable }\end{array}$ & -1.9 & 0.5 & -1.4 & -3.5 & -3.4 & -1.3 & -0.4 & -0.1 \\
\hline Primary balance (including grants) ${ }^{1}$ & -5.3 & -3.2 & -2.4 & 0.9 & -1.3 & -2.6 & -1.5 & -1.0 \\
\hline Recurrent domestic balance ${ }^{2}$ & 2.2 & 6.1 & 3.8 & 3.0 & 3.3 & 4.9 & 4.6 & 5.0 \\
\hline Net external flows ${ }^{3}$ & 12.5 & 8.6 & 9.8 & 1.1 & 8.5 & 8.0 & 7.4 & 7.7 \\
\hline Domestic debt (including arrears and accounts & 28.4 & 293 & 220 & & 229 & 190 & 171 & 153 \\
\hline Nominal GDP (Millions of Escudos) & 98,139 & 103,867 & 115,506 & 115,506 & 115,506 & 130,416 & 145,409 & 161,611 \\
\hline
\end{tabular}

Sources: Ministry of Finance and Public Administration, Bank of Cape Verde, and IMF staff estimates and projections.

${ }^{1}$ Overall balance (including grants) - total expenditure + domestic and external interest payments.

${ }^{2}$ Domestic revenue - recurrent expenditure.

${ }^{3}$ External grants + net foreign financing.

${ }^{4}$ The capital expenditure budget is typically underexecuted.

${ }^{5}$ The energy subsidies recorded in 2007 were incurred in previous periods.

${ }^{6}$ Reflects borrowing from the pension fund. 
Table 4. Cape Verde: Balance of Payments, 2005-10

(Millions of Cape Verde escudos, unless otherwise indicated)

\begin{tabular}{|c|c|c|c|c|c|c|}
\hline \multirow[b]{3}{*}{ Current account balance (including official transfers) } & \multirow[t]{2}{*}{2005} & \multirow{2}{*}{$\frac{2006}{\text { stimates }}$} & 2007 & 2008 & 2009 & \multirow[t]{2}{*}{2010} \\
\hline & & & \multicolumn{3}{|c|}{ Projections } & \\
\hline & $-3,016$ & $-5,259$ & $-10,965$ & $-14,978$ & $-18,292$ & $-23,686$ \\
\hline Trade balance & $-30,960$ & $-40,654$ & $-54,809$ & $-65,429$ & $-78,040$ & $-90,926$ \\
\hline Exports, f.o.b. & 7,891 & 8,429 & 6,454 & 6,788 & 6,943 & 7,144 \\
\hline Imports, f.o.b. & $-38,851$ & $-49,083$ & $-61,263$ & $-72,218$ & $-84,983$ & $-98,069$ \\
\hline Services (net) & 6,148 & 13,822 & 21,244 & 27,204 & 32,991 & 38,535 \\
\hline Credit & 24,667 & 35,523 & 43,578 & 52,672 & 61,752 & 70,798 \\
\hline Of which: tourism & 10,466 & 19,097 & 26,249 & 34,233 & 42,044 & 49,156 \\
\hline Debit & $-18,519$ & $-21,700$ & $-22,334$ & $-25,467$ & $-28,762$ & $-32,263$ \\
\hline Income (net) & $-2,984$ & $-3,952$ & $-3,149$ & $-3,599$ & $-4,204$ & $-4,934$ \\
\hline Credit & 1,641 & 1,627 & 1,923 & 2,285 & 2,623 & 2,993 \\
\hline Debit & $-4,625$ & $-5,579$ & $-5,072$ & $-5,884$ & $-6,827$ & $-7,927$ \\
\hline Government interest & -551 & -522 & -531 & -604 & -687 & -753 \\
\hline Interest by other sectors & $-3,040$ & $-2,443$ & $-2,114$ & $-2,397$ & $-2,673$ & $-3,012$ \\
\hline Income on direct investment and other income & $-1,034$ & $-2,614$ & $-2,428$ & $-2,882$ & $-3,466$ & $-4,162$ \\
\hline Current transfers (net) & 24,780 & 25,525 & 25,750 & 26,846 & 30,961 & 33,639 \\
\hline Government & 4,090 & 4,310 & 5,565 & 3,584 & 5,293 & 5,662 \\
\hline Other & 20,690 & 21,215 & 20,185 & 23,261 & 25,668 & 27,976 \\
\hline Capital and financial account (net) & 7,031 & 10,871 & 19,178 & 20,779 & 24,537 & 29,040 \\
\hline Capital transfers & 1,821 & 1,540 & 2,199 & 2,841 & 3,361 & 4,213 \\
\hline Government & 1,831 & 1,540 & 2,199 & 2,841 & 3,361 & 4,213 \\
\hline Other & 200 & 0 & 0 & 0 & 0 & 0 \\
\hline Direct investment (net) & 6,696 & 9,722 & 10,164 & 14,346 & 17,158 & 20,363 \\
\hline Portfolio investment & 0 & 13 & 400 & 550 & 550 & 550 \\
\hline Government & 1,880 & 2,266 & 2,229 & 2,329 & 2,085 & 2,510 \\
\hline Trust Fund & -200 & 0 & 0 & 0 & 0 & 0 \\
\hline Net official flows & 2,080 & 2,266 & 2,229 & 2,329 & 2,085 & 2,510 \\
\hline Disbursements & 4,314 & 4,240 & 4,176 & 4,403 & 4,321 & 4,697 \\
\hline Amortization & $-2,234$ & $-1,974$ & $-1,947$ & $-2,074$ & $-2,236$ & $-2,187$ \\
\hline Other & 0 & 0 & 0 & 0 & 0 & 0 \\
\hline Other investments & $-3,366$ & $-2,670$ & 4,186 & 713 & 1,382 & 1,403 \\
\hline Commercial banks & $-4,883$ & $-2,543$ & 1,823 & -638 & 19 & 20 \\
\hline Commercial credit (net) & 564 & 189 & 0 & 0 & 0 & 0 \\
\hline Other & 953 & -316 & 2,363 & 1,351 & 1,363 & 1,384 \\
\hline Net errors and omissions & 986 & -647 & 0 & 0 & 0 & 0 \\
\hline Overall balance & 5,000 & 4,965 & 8,225 & 5,802 & 6,239 & 5,348 \\
\hline Financing & $-5,000$ & $-4,965$ & $-8,225$ & $-5,802$ & $-6,239$ & $-5,348$ \\
\hline Gross international reserves (- accumulation) & $-4,964$ & $-5,044$ & $-8,225$ & $-5,802$ & $-6,239$ & $-5,348$ \\
\hline Of which: IMF (net) & 380 & -96 & -37 & -62 & -123 & -184 \\
\hline Exceptional financing & -36 & 78 & 0 & 0 & 0 & 0 \\
\hline Financing gap & 0 & 0 & 0 & 0 & 0 & 0 \\
\hline \multicolumn{7}{|l|}{ Memorandum items: } \\
\hline Current account (including official transfers; percent of GDP) & -3.4 & -5.1 & -9.5 & -11.5 & -12.6 & -14.7 \\
\hline Current account (excluding official transfers; percent of GDP) & -8.0 & -9.2 & -14.3 & -14.2 & -16.2 & -18.2 \\
\hline Overall balance (percent of GDP) & 5.6 & 4.8 & 7.1 & 4.4 & 4.3 & 3.3 \\
\hline Gross international reserves & 16,260 & 21,304 & 29,529 & 35,331 & 41,570 & 46,917 \\
\hline Months of current year's import of goods and services & 3.4 & 3.6 & 4.2 & 4.3 & 4.4 & 4.3 \\
\hline Months of next year's import of goods and services & 2.8 & 3.1 & 3.6 & 3.7 & 3.8 & 3.9 \\
\hline External public debt & 47,954 & 49,869 & 52,098 & 52,364 & 54,153 & 56,222 \\
\hline External aid (grants and loans; percent of GDP) & 11.5 & 9.7 & 10.3 & 8.3 & 8.9 & 9.0 \\
\hline
\end{tabular}

Sources: Bank of Cape Verde; and IMF staff estimates and projections. 
Table 5. Cape Verde: Monetary Survey, 2005-10

\begin{tabular}{|c|c|c|c|c|c|c|c|}
\hline & \multirow{3}{*}{$\begin{array}{l}2005 \\
\text { Dec. }\end{array}$} & \multirow{2}{*}{\multicolumn{2}{|c|}{$\begin{array}{l}2006 \\
\text { Dec. }\end{array}$}} & 2007 & 2008 & 2009 & 2010 \\
\hline & & & & \multicolumn{4}{|c|}{ Dec. } \\
\hline & & Program & Actual & \multicolumn{4}{|c|}{ Projections } \\
\hline & \multicolumn{6}{|c|}{ (Millions of Cape Verde escudos, unless otherwise specified) } & \\
\hline $\begin{array}{l}\text { Net foreign assets } \\
\text { Ot which: excluding the eftect ot a purchase of a }\end{array}$ & 21,889 & 26,056 & 25,138 & 31,577 & 38,079 & 44,421 & 49,934 \\
\hline Portuguese credit to Electra in 2006 & 21,889 & 26,056 & 29,532 & & & & \\
\hline Foreign assets & 27,549 & 31,716 & 31,285 & 38,687 & 45,248 & 51,590 & 57,043 \\
\hline Of which: foreign reserves & 16,260 & 18,356 & 21,304 & 29,529 & 35,331 & 41,570 & 46,917 \\
\hline Foreign liabilities & $-5,660$ & $-5,660$ & $-6,147$ & $-7,111$ & $-7,169$ & $-7,169$ & $-7,109$ \\
\hline Net domestic assets & 50,754 & 56,456 & 60,398 & 65,340 & 72,317 & 79,771 & 89,337 \\
\hline Net domestic credit & 60,193 & 65,127 & 70,487 & 75,472 & 82,492 & 89,988 & 99,599 \\
\hline Net claims on general government & 25,697 & 25,854 & 25,632 & 19,672 & 18,965 & 18,100 & 18,248 \\
\hline Claims on the Trust Fund (TCMFs) & 11,038 & 11,038 & 11,038 & 11,038 & 11,038 & 11,038 & 11,038 \\
\hline Net claims on the central government & 15,207 & 15,406 & 15,392 & 9,433 & 8,726 & 7,861 & 8,014 \\
\hline Credit to central government & 20,044 & 21,033 & 19,470 & 14,916 & 14,110 & 13,151 & 13,215 \\
\hline Deposits of central government & $-4,837$ & $-5,626$ & $-4,078$ & $-5,483$ & $-5,384$ & $-5,290$ & $-5,200$ \\
\hline Ot which: project deposits & -351 & -277 & -716 & -277 & -277 & -277 & -277 \\
\hline Net claims on local government & 21 & -22 & -311 & -311 & -312 & -312 & -312 \\
\hline Net claims on other government agencies (INPS) & -569 & -569 & -488 & -488 & -488 & -488 & -493 \\
\hline Credit to the economy & 34,496 & 39,273 & 44,855 & 55,800 & 63,527 & 71,888 & 81,351 \\
\hline \multicolumn{8}{|l|}{$\begin{array}{l}\text { Ot which: excluding purchase ot a Portuguese } \\
\text { credit to Electra and subsequent off loading }\end{array}$} \\
\hline on the domestic securities market & 34,496 & 39,273 & 40,461 & & & $\ldots$ & \\
\hline Credit to public enterprises & 431 & 497 & 634 & 678 & 703 & 736 & 770 \\
\hline Credit to private sector & 34,040 & 38,751 & 44,205 & 55,106 & 62,808 & 71,137 & 80,565 \\
\hline Claims on nonbank financial institutions & 26 & 26 & 16 & 16 & 16 & 16 & 16 \\
\hline Other items (net) & $-9,439$ & $-8,671$ & $-10,089$ & $-10,133$ & $-10,175$ & $-10,218$ & $-10,261$ \\
\hline Broad money (M2) & 72,643 & 82,511 & 85,536 & 96,916 & 110,396 & 124,192 & 139,271 \\
\hline Narrow money (M1) & 28,718 & 32,620 & 35,860 & 40,631 & 46,282 & 52,066 & 58,387 \\
\hline Currency outside banks & 7,634 & 8,464 & 7,731 & 8,760 & 9,978 & 11,225 & 12,588 \\
\hline Demand deposits & 21,084 & 24,156 & 28,129 & 31,871 & 36,304 & 40,841 & 45,799 \\
\hline Quasimoney & 40,566 & 46,076 & 45,505 & 51,559 & 58,730 & 66,069 & 74,091 \\
\hline Time deposits & 38,390 & 43,605 & 43,334 & 49,100 & 55,929 & 62,918 & 70,558 \\
\hline Other quasimonetary deposits & 2,176 & 2,471 & 2,170 & 2,459 & 2,801 & 3,151 & 3,534 \\
\hline \multirow[t]{2}{*}{ Foreign currency deposits } & 3,359 & 3,815 & 4,172 & 4,727 & 5,384 & 6,057 & 6,792 \\
\hline & \multicolumn{7}{|c|}{ (Change in percent of broad money 12 months earlier) } \\
\hline Net foreign assets & 12.9 & 5.7 & 4.5 & 7.5 & 6.7 & 5.7 & 4.4 \\
\hline Net domestic assets & 2.7 & 7.8 & 13.3 & 5.8 & 7.2 & 6.8 & 7.7 \\
\hline Net domestic credit & 4.6 & 7.9 & 14.2 & 5.8 & 7.2 & 6.8 & 7.7 \\
\hline Net claims on the central government & 0.2 & 1.4 & 0.3 & -7.0 & -0.7 & -0.8 & 0.1 \\
\hline Credit to the economy & 4.6 & 6.5 & 14.3 & 12.8 & 8.0 & 7.6 & 7.6 \\
\hline Credit to public enterprises & 0.4 & 0.0 & 0.3 & 0.1 & 0.0 & 0.0 & 0.0 \\
\hline Credit to private sector & 4.2 & 6.5 & 14.0 & 12.7 & 7.9 & 7.5 & 7.6 \\
\hline Other items (net) & -1.9 & 0.0 & -0.9 & -0.1 & 0.0 & 0.0 & 0.0 \\
\hline \multicolumn{8}{|l|}{ Selected monetary indicators: } \\
\hline Income velocity of money & 1.32 & 1.27 & 1.31 & 1.27 & 1.26 & 1.24 & 1.23 \\
\hline Emigrant deposits & 28,318 & 31,339 & 31,293 & 35,457 & 40,388 & 45,436 & 50,953 \\
\hline Excess reserves /total deposits (percent) & 2.1 & 0.8 & 1.3 & 1.5 & 1.4 & 1.4 & 1.3 \\
\hline Money multilplier (M2/M0) & 3.44 & 3.69 & 3.92 & 4.19 & 4.19 & 4.18 & 4.19 \\
\hline Credit to the economy (percentage change) & 9.0 & 13.8 & 30.0 & 24.4 & 13.8 & 13.2 & 28.1 \\
\hline
\end{tabular}

Sources: Bank of Cape Verde, and IMF staff estimates and projections. 
Table 6. Cape Verde: Central Bank Survey, 2005-10

\begin{tabular}{|c|c|c|c|c|c|c|c|}
\hline & \multirow{3}{*}{$\begin{array}{l}2005 \\
\text { Dec. }\end{array}$} & \multirow{2}{*}{\multicolumn{2}{|c|}{$\begin{array}{l}2006 \\
\text { Dec. }\end{array}$}} & 2007 & 2008 & 2009 & 2010 \\
\hline & & & & \multicolumn{4}{|c|}{ Dec. } \\
\hline & & Program & Actual & \multicolumn{4}{|c|}{ Projections } \\
\hline & \multicolumn{6}{|c|}{ (Millions of Cape Verde escudos, unless otherwise specified) } & \\
\hline Net foreign assets & 15,308 & 17,403 & 20,390 & 28,652 & 34,516 & 40,877 & 46,409 \\
\hline Of which: net international reserves & 15,109 & 17,204 & 20,216 & 28,477 & 34,341 & 40,703 & 46,235 \\
\hline Foreign assets & 16,523 & 18,618 & 21,536 & 29,761 & 35,563 & 41,802 & 47,149 \\
\hline Foreign liabilities & $-1,215$ & $-1,215$ & $-1,146$ & $-1,109$ & $-1,047$ & -924 & -740 \\
\hline Net domestic assets & 5,828 & 4,965 & 1,454 & $-5,517$ & $-8,141$ & $-11,199$ & $-13,131$ \\
\hline Net domestic credit & 7,678 & 6,047 & 3,518 & $-3,412$ & $-5,994$ & $-9,009$ & $-10,898$ \\
\hline Trust Fund claims & 4,605 & 4,605 & 4,605 & 4,605 & 4,605 & 4,605 & 4,605 \\
\hline Net claims on central government & 1,882 & 1,093 & 1,748 & 239 & 239 & 239 & 239 \\
\hline Credit to central government & 4,779 & 4,779 & 3,739 & 3,739 & 3,739 & 3,739 & 3,739 \\
\hline Deposits of central government & $-2,896$ & $-3,685$ & $-1,990$ & $-3,500$ & $-3,500$ & $-3,500$ & $-3,500$ \\
\hline Of which: project accounts & -351 & -277 & -716 & -277 & -277 & -277 & -277 \\
\hline Ot which: toreign currency deposits & $-1,347$ & $-1,347$ & -682 & -682 & -682 & -682 & -682 \\
\hline Claims on local government & 0 & 0 & 0 & 0 & 0 & 0 & 0 \\
\hline Credit to the economy & 1,163 & 1,163 & 1,147 & 1,269 & 1,426 & 1,583 & 1,754 \\
\hline Credit to public enterprises & 54 & 54 & 47 & 47 & 47 & 47 & 47 \\
\hline Credit to private sector & 1,094 & 1,094 & 1,091 & 1,213 & 1,370 & 1,527 & 1,698 \\
\hline Claims on nonbank financial institutions & 15 & 15 & 9 & 9 & 9 & 9 & 9 \\
\hline Credit to commercial banks & 27 & -815 & $-3,983$ & $-9,525$ & $-12,264$ & $-15,436$ & $-17,495$ \\
\hline Other items (net) & $-1,850$ & $-1,082$ & $-2,063$ & $-2,104$ & $-2,147$ & $-2,189$ & $-2,233$ \\
\hline Assets & 2,481 & 1,442 & 2,791 & 2,847 & 2,904 & 2,962 & 3,021 \\
\hline Liabilities & 4,332 & 2,525 & 4,854 & 4,951 & 5,050 & 5,151 & 5,254 \\
\hline Reserve money (M0) & 21,136 & 22,368 & 21,845 & 23,135 & 26,375 & 29,678 & 33,278 \\
\hline Currency outside banks & 7,634 & 8,464 & 7,731 & 8,760 & 9,978 & 11,225 & 12,588 \\
\hline Cash in vaults & 1,058 & 1,205 & 1,457 & 1,168 & 1,330 & 1,497 & 1,678 \\
\hline Deposits of commercial banks & 12,443 & 12,697 & 12,654 & 13,205 & 15,064 & 16,954 & 19,009 \\
\hline Deposits of private sector & 0 & 0 & 0 & 0 & 0 & 0 & 0 \\
\hline Deposits of other financial institutions & 2 & 2 & 3 & 3 & 3 & 3 & 3 \\
\hline Gross international reserves (millions of euros) & 147.4 & 166.4 & 193.1 & 267.7 & 320.3 & 376.9 & 425.4 \\
\hline Net international reserves (millions of euros) & 137.0 & 156.0 & 183.3 & 258.2 & 311.3 & 369.0 & 419.2 \\
\hline Reserve money (12-month change in percent) & 14.3 & 5.8 & 3.4 & 5.9 & 14.0 & 12.5 & 12.1 \\
\hline
\end{tabular}

Sources: Bank of Cape Verde, and IMF staff estimates and projections. 
Table 7. Cape Verde: Deposit Money Bank Survey, 2005-10

\begin{tabular}{|c|c|c|c|c|c|c|c|}
\hline & \multirow{3}{*}{$\begin{array}{l}2005 \\
\text { Dec. }\end{array}$} & \multirow{2}{*}{\multicolumn{2}{|c|}{$\begin{array}{l}2006 \\
\text { Dec. }\end{array}$}} & 2007 & 2008 & 2009 & 2010 \\
\hline & & & & \multicolumn{4}{|c|}{ Dec. } \\
\hline & & Program & Actual & \multicolumn{4}{|c|}{ Projections } \\
\hline & \multicolumn{6}{|c|}{ (Millions of Cape Verde escudos, unless otherwise specified) } & \\
\hline $\begin{array}{l}\text { Net foreign assets } \\
\text { Of which: excluding the effect of a purchase of a }\end{array}$ & 6,581 & 8,652 & 4,747 & 2,925 & 3,563 & 3,544 & 3,524 \\
\hline Portuguese credit to Electra & 6,581 & 8,652 & 8,315 & $\ldots$ & $\ldots$ & & \\
\hline Foreign assets & 11,026 & 13,098 & 9,749 & 8,927 & 9,685 & 9,788 & 9,894 \\
\hline Foreign liabilities & $-4,445$ & $-4,445$ & $-5,002$ & $-6,002$ & $-6,122$ & $-6,244$ & $-6,369$ \\
\hline Of which: nonresident deposits & $-3,520$ & $-3,766$ & $-4,214$ & $-5,057$ & $-5,158$ & $-5,261$ & $-5,366$ \\
\hline Net domestic assets & 58,427 & 65,395 & 73,055 & 85,232 & 96,854 & 109,423 & 123,159 \\
\hline Net domestic credit & 66,016 & 72,982 & 81,080 & 93,257 & 104,880 & 117,448 & 131,184 \\
\hline Net claims on general government & 19,209 & 20,156 & 19,279 & 14,829 & 14,122 & 13,256 & 13,404 \\
\hline Trust Fund claims & 6,433 & 6,433 & 6,433 & 6,433 & 6,433 & 6,433 & 6,433 \\
\hline Other government deposits (INPS) & -569 & -569 & -488 & -488 & -488 & -488 & -493 \\
\hline Net claims on central government & 13,324 & 14,313 & 13,644 & 9,195 & 8,488 & 7,623 & 7,776 \\
\hline Treasury bonds & 9,173 & 9,847 & 10,030 & 5,221 & 4,095 & 2,711 & 2,288 \\
\hline Treasury bills & 5,609 & 5,889 & 5,086 & 5,340 & 5,661 & 6,085 & 6,572 \\
\hline Oher credits & 484 & 518 & 616 & 616 & 616 & 616 & \\
\hline Deposits of central government & $-1,941$ & $-1,941$ & $-2,088$ & $-1,983$ & $-1,884$ & $-1,790$ & $-1,700$ \\
\hline Net claims on local government & 21 & -22 & -311 & -311 & -312 & -312 & -312 \\
\hline Claims on local government & 276 & 236 & 271 & 277 & 282 & 288 & 294 \\
\hline Deposits of local government & -256 & -258 & -582 & -588 & -594 & -600 & -606 \\
\hline \multicolumn{8}{|l|}{$\begin{array}{l}\text { Of which: excluding purchase of a Portuguese } \\
\text { credit to Electra and subsequent off loading }\end{array}$} \\
\hline on the domestic securities market & 33,333 & 38,110 & 39,314 & & & & \\
\hline Credit to public enterprises & 377 & 443 & 587 & 631 & 656 & 689 & 724 \\
\hline Credit to private sector & 32,945 & 37,656 & 43,114 & 53,893 & 61,438 & 69,609 & 78,867 \\
\hline Claims on nonbank financial institutions & 11 & 11 & 6 & 6 & 6 & 6 & 6 \\
\hline Net claims on the Bank of Cape Verde & 13,473 & 14,717 & 18,094 & 23,898 & 28,658 & 33,887 & 38,183 \\
\hline Total reserves & 13,500 & 13,902 & 14,111 & 14,373 & 16,394 & 18,451 & 20,687 \\
\hline Vault cash & 1,058 & 1,205 & 1,457 & 1,168 & 1,330 & 1,497 & 1,678 \\
\hline Deposits with central bank & 12,443 & 12,697 & 12,654 & 13,205 & 15,064 & 16,954 & 19,009 \\
\hline Required reserves & 11,051 & 12,117 & 11,670 & 11,914 & 13,643 & 15,413 & 17,344 \\
\hline Excess reserves & 1,391 & 579 & 984 & 1,291 & 1,420 & 1,541 & 1,665 \\
\hline Credit to the Bank of Cape Verde & -27 & 815 & 3,983 & 9,525 & 12,264 & 15,436 & 17,495 \\
\hline Other items (net) & $-7,589$ & $-7,587$ & $-8,026$ & $-8,026$ & $-8,026$ & $-8,026$ & $-8,026$ \\
\hline Deposit liabilities to nonbank residents & 65,008 & 74,047 & 77,802 & 88,156 & 100,418 & 112,967 & 126,683 \\
\hline Local currency deposits & 61,649 & 70,232 & 73,631 & 83,430 & 95,034 & 106,910 & 119,891 \\
\hline Demand deposits & 21,083 & 24,156 & 28,126 & 31,871 & 36,304 & 40,841 & 45,799 \\
\hline Ot which: emigrant deposits & 3,394 & 3,756 & 4,043 & 4,581 & 5,218 & 5,870 & 6,583 \\
\hline Quasimoney & 40,566 & 46,076 & 45,505 & 51,559 & 58,730 & 66,069 & 74,091 \\
\hline Time deposits & 38,390 & 43,605 & 43,334 & 49,100 & 55,929 & 62,918 & 70,558 \\
\hline Of which: emigrant deposits & 23,825 & 26,367 & 26,148 & 29,627 & 33,748 & 37,965 & 42,575 \\
\hline Other quasimonetary deposits & 2,176 & 2,471 & 2,170 & 2,459 & 2,801 & 3,151 & 3,534 \\
\hline Foreign currency deposits & 3,359 & 3,815 & 4,172 & 4,727 & 5,384 & 6,057 & 6,792 \\
\hline Of which: emigrant deposits & 1,100 & 1,217 & 1,102 & 1,249 & 1,423 & 1,600 & 1,795 \\
\hline \multicolumn{8}{|l|}{ Memorandum items: } \\
\hline Emigrant deposits (as ratio to total deposits) & 0.44 & 0.38 & 0.40 & 0.40 & 0.40 & 0.40 & 0.37 \\
\hline Other deposits (as ratio to total deposits) & 0.61 & 0.62 & 0.63 & 0.63 & 0.63 & 0.63 & 0.63 \\
\hline Composition of emigrant deposits & 1.00 & 1.00 & 1.00 & 1.00 & 1.00 & 1.00 & 1.00 \\
\hline Local currency & 0.96 & 0.96 & 0.96 & 0.96 & 0.96 & 0.96 & 0.96 \\
\hline Demand & 0.12 & 0.12 & 0.13 & 0.13 & 0.13 & 0.13 & 0.13 \\
\hline Time & 0.84 & 0.84 & 0.84 & 0.84 & 0.84 & 0.84 & 0.84 \\
\hline Foreign currency & 0.04 & 0.04 & 0.04 & 0.04 & 0.04 & 0.04 & 0.04 \\
\hline
\end{tabular}

Sources: Bank of Cape Verde, and IMF staff estimates and projections. 
Table 8. Cape Verde: Proposed Work Program 2008-09

\begin{tabular}{lll}
\hline Mission Date & Purpose & Board Review \\
\hline February 2008 & $\begin{array}{l}\text { Discussions on the fourth review } \\
\text { against end-December 2007 } \\
\text { assessment criteria }\end{array}$ & End-April 2008 \\
August 2008 & $\begin{array}{l}\text { Discussions on the fifth review } \\
\text { against end-June 2008 assessment } \\
\text { criteria }\end{array}$ & End-October 2008 \\
February 2009 & $\begin{array}{l}\text { Discussions on the sixth review } \\
\text { against end-December 2008 } \\
\text { assessment criteria }\end{array}$ & End-April 2009 \\
\hline
\end{tabular}




\section{APPENDIX I-LETTER OF INTENT}

November 29, 2007

Mr. Dominique Strauss-Kahn

Managing Director

International Monetary Fund

$70019^{\text {th }}$ Street N.W.

Washington DC 20431

USA

Dear Mr. Strauss-Kahn:

The attached Memorandum of Economic and Financial Policies, reviews implementation to date of the Cape Verde government's macroeconomic and structural program under the country's three-year Policy Support Instrument (PSI), approved by the IMF Executive Board in July 2006. Details of this program are set out in the initial Memorandum of Economic and Financial Policies (MEFP) of July 2006, which was updated in January 2007 and May 2007 in conjunction with the first and second reviews of the program. The attached MEFP builds on the initial memorandum, with particular attention to policy developments and prospects for 2008. The fourth and fifth reviews of the PSI are scheduled to be completed by end-April 2008 and end-October 2008, respectively.

The government believes that this program will provide firm support for macroeconomic stability and the exchange rate peg, and create the fiscal space needed to adjust to possible declines in concessional external financing following the country's expected graduation from Least-Developed-Country status in 2008.

Under the PSI, the government will keep the IMF regularly updated on economic and policy developments and will provide the data needed for adequate monitoring of the program, including in the context of the twice-yearly reviews. During the period of the PSI, Cape Verde will consult with the IMF on the adoption of any measures that may be appropriate at the initiative of the government or whenever the Managing Director of the IMF requests such a consultation.

We authorize the IMF to publish this letter, the attached MEFP, and the related staff report.

Sincerely yours

$/ \mathrm{s} /$

Cristina Duarte

Minister of Finance and Public Administration. 


\section{Attachment I-Memorandum of Economic and Financial Policies}

\section{INTRODUCTION AND BACKGROUND}

1. Cape Verde's economic performance remains strong. The outlook for growth and inflation is favorable, and fiscal and monetary policies are consistent with program goals. In particular, public debt is coming down as a share of GDP and international reserves are building rapidly. Important progress is also being made toward the structural objectives of the program namely public sector management, especially fiscal accounting, budget execution and control, and arrears prevention; strengthening of the financial sector and improving energy sector regulation.

\section{Reflecting this solid performance, Cape Verde is on the verge of graduation} from the UN least-developed-country (LDC) status in early 2008. While this graduation has its benefits, the government recognizes that this will also entail challenges. In particular, while the government of Cape Verde will continue to seek concessional support, we need to prepare to become less dependent on aid in the future. Further improvements in the business environment are needed to attract private capital. At the same time, to support macroeconomic stability and the exchange rate peg, we intend to reduce government's domestic debt ratio, increase foreign reserves and create fiscal space to accommodate possible declines in concessional external financing as the country graduates from LDC status.

3. In this vein, the government's policies will continue to focus on four areas to enhance macroeconomic stability and implement structural reforms that remove barriers to productivity growth and encourage private-sector led development. Specifically we will aim to:

- $\quad$ Consolidate macroeconomic stability, notably by reducing public debt and increasing official foreign exchange reserves;

- $\quad$ Improve public sector financial management, including by strengthening macroeconomic coordination and planning, stopping arrears, broadening the tax base, and improving internal and external audit processes;

- $\quad$ Reduce fiscal risks, including those that could arise from operations of state-owned enterprises; and

- $\quad$ Strengthen regulation and supervision of the financial sector to enhance the efficiency of the financial system, particularly in the growing offshore financial center. 


\section{The Current Economic Setting}

\section{Cape Verde's recent economic indicators point to high growth, falling} unemployment and modest inflation. According to new data from the National Statistical Institute (INE), growth in 2006 appears to have been much stronger than earlier estimatedreaching $10 \mathrm{3} / 4$ percent. Growth was particularly strong in tourism, telecommunications, financial services, and construction. In line with this, growth for 2007 is projected to be around 7 percent. "Headline" consumer price inflation has largely come down as expected since 2006, but is now being pushed up temporarily due to poor rainfall and recent adjustments in petroleum prices. Consumer price inflation (annual average) is now projected to reach about 5 percent for 2007.

\section{The June 2007 program quantitative targets were observed by wide margins.}

- International reserves continue to accumulate rapidly, significantly exceeding the program target at end-June. Cape Verde has continued to attract official assistance, and tourism and foreign direct investment (FDI) flows are both surging.

- Domestic borrowing was significantly below its ceiling. The government has already unwound the CVEsc 2.3 billion higher-than-targeted borrowing that arose in 2006, and the end-2007 domestic borrowing ceiling has been lowered by the same amount. Tax revenues are growing higher than projected, and receipts from privatization and land sales have already surpassed the amounts budgeted for 2007 . Expenditure commitments are being closely monitored and controlled, with expenditures evolving in line with budget ceilings. The government is further strengthening budget management to ensure that this strong performance continues (see below). Because of these measures and stronger growth prospects, domestic debt is expected to decline to below 23 percent of GDP by end-2007, significantly below the target set at the beginning of the PSI program.

6. Significant progress has been made on the reform front. In the area of public sector financial management, the government has taken firm measures to prevent a recurrence of central government payment arrears, and there has been no accumulation of such arrears in 2007. There has also been significant reduction in the accumulation of arrears by municipalities (with most of them being current). We are also strengthening budget execution and monitoring practices, including improved control of end-year spending commitments. The Bank of Cape Verde (BCV) is moving ahead with plans to strengthen banking supervision, to upgrade legislation concerning anti-money laundering and combating the financing of terrorism (AML/CFT), and to establish a financial intelligence unit. As part of its broader strategy to improve efficiency and capacity in the energy sector, the government will introduce more transparent approaches for pricing energy products, which will help to ensure that no further contingent fiscal liabilities are borne by the budget. 


\section{MaCroeconomic ObJectives ANd Policies for 2008}

\section{A. Macroeconomic Objectives}

7. We expect growth to be about $73 / 4$ percent in 2008 , underpinned by further growth in tourism investment and other services, and by public infrastructure investment. Inflation is expected to return to trend value of about 2 percent consistent with the peg. Gross reserves are targeted to increase by 0.1 month of imports. The specific program objectives for the remainder of 2007 and for 2008 are set out in Table 1 (attached).

\section{B. Fiscal policy}

8. Continued fiscal consolidation remains key to our macroeconomic program and the 2008 budget aims at a further sizable reduction of the domestic debt-to-GDP ratio. This budget is based on prudent projections for revenues and expenditures, with tax revenues and the wage bill budgeted to grow by less than nominal GDP. The goods and services budget is sufficient to ensure timely payment of recurrent bills, notably electricity, water, and fuel. To provide more flexibility in managing debt, the budget's ceiling for domestic borrowing will be on a net basis (rather than on a gross basis as in the past), and the Treasury will seek to clear arrears more rapidly if extraordinary revenues materialize. There will be no additional net domestic borrowing by the budget in 2008. Consequently, the net domestic debt-to-GDP ratio is projected to decline to below 20 percent by end-2008, one year ahead of the original target set under the PSI.

\section{Monetary Management}

9. Monetary management remains fully consistent with the program goal of building up foreign exchange reserves to support the exchange rate peg to the Euro. Within the framework of the exchange rate peg, the $\mathrm{BCV}$ will continue to more actively manage liquidity and interest rate differentials with the Euro area. Liquidity management will continue to be implemented through the issuance of 14-day and 6-month central bank bills and the interest rate offered on the 14-day bill constitutes the BCV's official policy rate. More generally, the BCV will continue to monitor the liquidity situation closely and its implications for domestic interest rates and credit growth, and adjust policy operations as appropriate.

\section{Structural Reform Issues}

\section{Public sector financial management:}

10. The government is moving ahead with its program of strengthening public sector financial management. In particular, the Ministry of Finance is: 
- $\quad$ Closely monitoring spending commitments, payments, and accounts payable through the SIGOF (Integrated Online Budget Management) system, which will be expanded to include all semi-autonomous institutes and most municipalities by the end of this year. In addition a new procedures manual will be drafted with Millennium Challenge Corporation (MCC) funding to support the ongoing decentralization of budget execution and the upcoming implementation of the new budget framework law and new chart of public accounts, and to ensure that all spending units will use SIGOF consistently;

- Instituting as of 2007 an earlier (November 30) deadline for granting new spending commitments to reduce end of year payment pressures. Spending commitments after November 30 will be authorized only on an exceptional basis;

- Introducing a formal mid-year review of fiscal developments: this will assess revenue, expenditure, and financing developments halfway through the fiscal year, compare these with budget forecasts, and take corrective actions if necessary.

- A new chart of government accounts (PNCP) has been approved by Parliament and will be implemented in 2008 .

- $\quad$ Preparing a new treasury management system;

- $\quad$ Moving ahead with the development of a medium-term expenditure framework.

11. The government intends to update and strengthen the tax framework. Drafts of the revised General Tax Code, a new Code on Judicial Processes, a Forced Tax Collection code, and bills covering individual and corporate income taxes (IRC and IRS) have been prepared, and these will be submitted to the National Assembly before end-December 2007. In addition, to further reduce and streamline the granting of tax incentives and exemptions, the government has commenced preparation of a unified law on tax benefits that will revoke all tax benefits not specifically mentioned in the new law. In support of this effort, the government requests further IMF technical assistance to meet its policy goals in this area and drafting of the associated legislation. The government intends to submit this legislation to the National Assembly in 2008. Pending the completion of this work, a moratorium has been put in place on the granting of new tax exemptions.

\section{To further improve tax administration, the government is strengthening the structure, functioning, and management of the General Directorate of Taxes (DGCI).} These reforms, which draw on the recommendations of recent technical assistance from the IMF, include refocused tax collection and control towards major tax payers. To deter tax evasion, the DGCI will cross-check, using computer tools, taxpayer information in databases in the Ministry of Labor, Social Security Institute and Customs. With the hiring of new tax inspectors, taxpayers will be audited more frequently. The DGCI is also preparing to 
implement the Forced Tax Collection Code as soon as possible after its approval. The recovery of tax liabilities of large amounts and close to expiry will be given priority. Recovery processes will be opened more quickly, and assets seized in these processes will be auctioned with minimal delay.

13. The Ministry of Finance has introduced firm measures to prevent accumulation of public sector arrears. In particular, the Ministry is:

- $\quad$ carefully monitoring the payment arrears of central government entities, including semi-autonomous agencies;

- $\quad$ working with these entities to strengthen their budget execution and payment practices; and

- $\quad$ applying procedures to make the required payments on behalf of these entities if they incur arrears and deduct those amounts from government transfers to them.

14. Furthermore, municipalities have agreed in principle to pay electricity bills on time, and are working with Electra to ensure that the amounts billed correctly reflect their responsibilities in this regard. The government is preparing a tax on consumer electricity bills to meet the costs of public lighting, which has been a persistent area of dispute between municipalities and Electra.

15. The government is strengthening external and internal audit processes. A new draft law concerning the Court of Auditors (TdC) has been submitted to the National Assembly. In addition, a new customs audit court has been set up and the Tax Inspectorate will be strengthened and modernized. As for internal audit, a new budget framework law will create the positions of financial controllers in 2008 to strengthen internal audit.

\section{The government is also preparing to implement a number of civil service} reforms. The draft Civil Service Framework Law is expected to be sent to the National Assembly and come into force in 2008. The key objectives of this law were described in the LOI for the second review of the PSI.

\section{Financial Sector Issues:}

17. The BCV is strengthening regulation and supervision of the financial sector. In particular:

- $\quad$ New bank regulations on capital ratios, provisioning and loan classification; credit risk assessment, and credit concentration have been approved and will soon be published; 
- New banking accounting rules based on international accounting standards will be implemented from 2008;

- $\quad$ Procedures for bank licensing and supervision are being strengthened, supported by technical assistance from the IMF;

- $\quad$ The BCV is finalizing information sharing agreements with the home country supervisor of most of the current offshore financial institutions, and seeking similar agreements with other countries;

- $\quad$ Following the recent AML/CFT assessment by the Fund, the BCV is preparing to implement the recommendations arising from this assessment in the first half of 2008 (a new structural benchmark under the program);

- $\quad$ Also drawing on the AML/CFT recommendations, legislation to set up a Financial Intelligence Unit is being prepared and will be presented to the National Assembly by end-March 2008 (a structural benchmark).

18. The above measures to strengthen the financial sector will support the BCV's strategy of further opening the capital account. This move will largely validate the current de facto situation regarding inward and outward capital flows. Measured liberalization will support the ongoing economic and financial development of Cape Verde, including access to international financial markets. A revised decree law to further liberalize the capital account has been drafted and should soon be submitted to the Government for approval.

\section{Energy Sector Reform}

19. The government is putting in place a comprehensive strategy to ensure that the energy sector is able to support the growth and development of Cape Verde, and is not a source of fiscal risk. Working with public and private participants in the sector, the government's strategy emphasizes:

- Investment to increase capacity and efficiency, including improvements in electricity generation and transmission, higher water production capacities, and development of alternative energy sources;

- Increased private sector participation in the sector's management and investment activities;

- Formation of a joint logistics company to handle importation, storage and inter-island distribution of oil products;

- Improvement in billing and collection practices, including use of pre-paid meters for electricity and reduction in illegal connections. 
20. To support the above developments, the government remains committed to improving the regulatory frameworks for the energy sector. With the administrative council of ARE (economic regulatory agency) again at full strength, the government is determined to ensure that domestic fuel prices are adjusted in line with international prices. In this respect, retail gasoline and diesel prices were increased by 15 and 11 percent respectively on October 24 which will clear the contingent fiscal liabilities that had built up over the period while prices were unchanged. Furthermore, the government has directed ARE to ensure, through regular fuel price adjustments, that no further fiscal liabilities accrue. To support the regular and transparent alignment of domestic fuel prices with international prices, ARE intends to have a new adjustment mechanism in place no later than end-March 2008. This mechanism will be based on international benchmark prices and applied with minimal delay after each shipment of oil imports. Publication and implementation of this mechanism will be a new structural benchmark under the PSI program.

\section{An adjustment in electricity and water tariffs is not required at present. A} commission charged by the oil companies on earlier fuel supplies to Electra (the electricity and water utility) should not have been charged. The resulting balance now owed to the Treasury will be drawn on to cover the difference between the (notional) revised fuel oil and the actual price Electra will be charged for the rest of 2007. After that the market price will be charged (with consequent adjustments in electricity and water prices).

\section{Implementation of the mechanism for setting base electricity tariffs is also} planned for the first quarter of 2008. This mechanism had originally been expected to be ready by June 2007, but has been delayed by the need to confirm two new members of the ARE administrative council. Its implementation by March 2008 (which will be an assessment criterion for the fourth review under the PSI) will enable base electricity tariffs to begin to reflect the broader structural reform strategy noted above that will improve efficiency in the energy sector. 


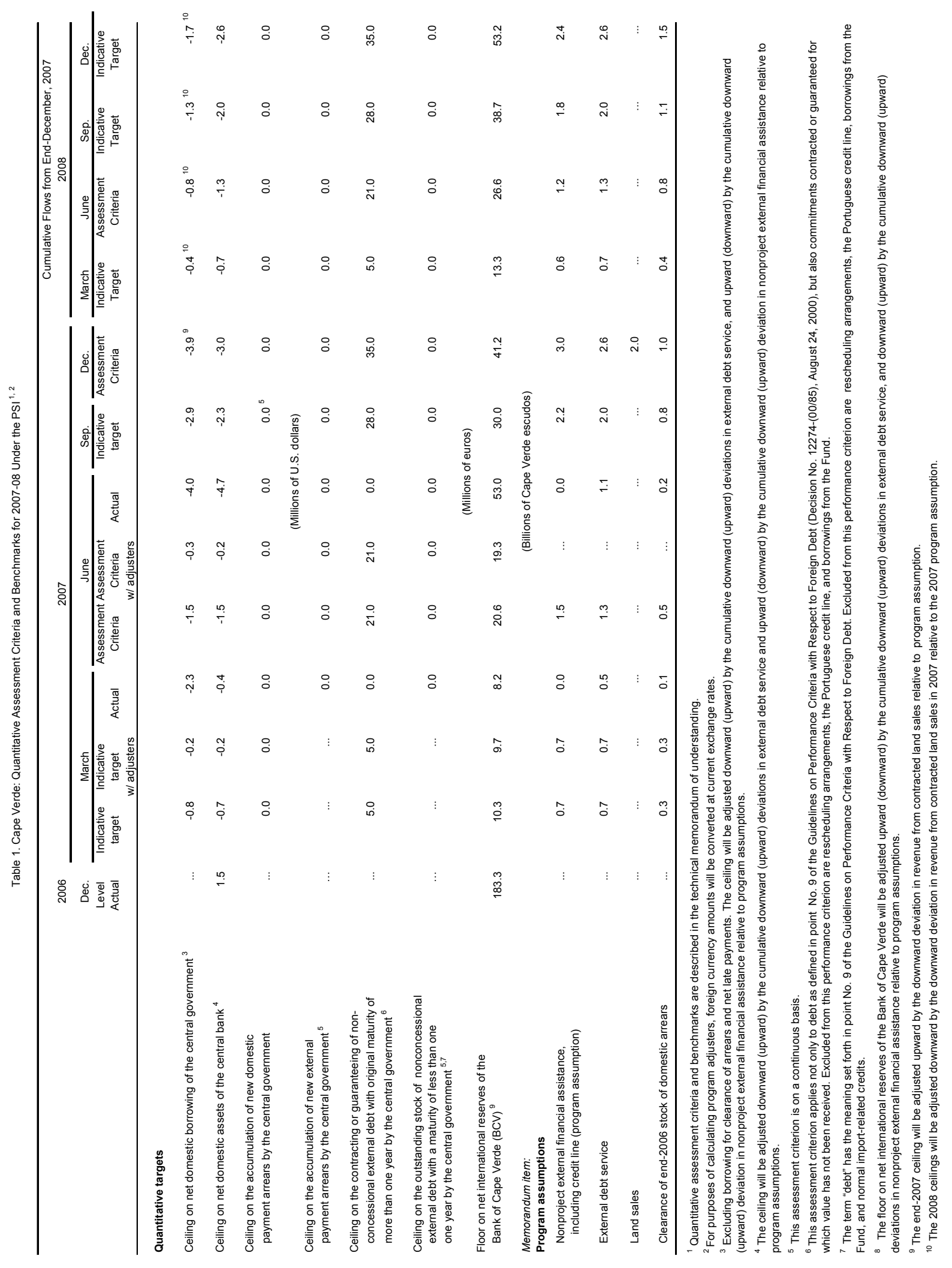


Table 2. Cape Verde: Structural Assessment Criteria and Benchmarks for 2007-08

\begin{tabular}{|c|c|c|c|}
\hline Objectives & Conditionality & Timing & Status \\
\hline & Structural Assessment Criteria & & \\
\hline Reduce fiscal risks. & $\begin{array}{l}\text { Fully implement the automatic utility } \\
\text { tariff adjustment mechanism. }\end{array}$ & End-March 2007 & Met \\
\hline Reduce fiscal risks. & $\begin{array}{l}\text { Finalize and publish the mechanism } \\
\text { for setting base utility tariffs }\end{array}$ & End-June 2007 & Not met \\
\hline \multirow[t]{2}{*}{ Reduce fiscal risks. } & $\begin{array}{l}\text { Fully apply mechanisms for setting } \\
\text { and adjusting electricity, water, and } \\
\text { fuel prices }\end{array}$ & $\begin{array}{l}\text { Continuous as of } \\
\text { July } 1,2007\end{array}$ & Not met \\
\hline & Structural Benchmarks & & \\
\hline $\begin{array}{l}\text { Strengthen financial } \\
\text { supervision. }\end{array}$ & $\begin{array}{l}\text { Sign formal information-sharing } \\
\text { agreements with home country } \\
\text { supervisors of subsidiaries and } \\
\text { branches operating in Cape Verde. }\end{array}$ & $\begin{array}{l}\text { End-December } \\
2006\end{array}$ & In progress \\
\hline $\begin{array}{l}\text { Improve budget } \\
\text { prioritization }\end{array}$ & Implement the MTEF. & End-June 2007 & In progress \\
\hline $\begin{array}{l}\text { Strengthen financial } \\
\text { regulation. }\end{array}$ & $\begin{array}{l}\text { Implement recommendations of the } \\
\text { task force on financial sector reform. }\end{array}$ & End-June 2007 & In progress \\
\hline $\begin{array}{l}\text { Strengthen fiscal } \\
\text { control. }\end{array}$ & $\begin{array}{l}\text { Implement laws to strengthen the } \\
\text { Court of Auditors (TdC) and the } \\
\text { National Chart of Public Accounts. }\end{array}$ & End-June 2007 & In preparation \\
\hline $\begin{array}{l}\text { Strengthen the tax } \\
\text { base. }\end{array}$ & $\begin{array}{l}\text { Large taxpayers unit to be fully } \\
\text { operational }\end{array}$ & End-June 2007 & Met \\
\hline $\begin{array}{l}\text { Strengthen tax } \\
\text { administration. }\end{array}$ & Finalize reform strategy for $\mathrm{DGCl}$ & $\begin{array}{l}\text { End-December } \\
2007\end{array}$ & \\
\hline $\begin{array}{l}\text { Strengthen the tax } \\
\text { base. }\end{array}$ & $\begin{array}{l}\text { Submit the new General Tax Code } \\
\text { to the National Assembly. }\end{array}$ & $\begin{array}{l}\text { End-December } \\
2007\end{array}$ & \\
\hline $\begin{array}{l}\text { Strengthen the tax } \\
\text { base. }\end{array}$ & $\begin{array}{l}\text { Submit the new Code on Judicial } \\
\text { Processes to the National Assembly. }\end{array}$ & $\begin{array}{l}\text { End-December } \\
2007\end{array}$ & \\
\hline $\begin{array}{l}\text { Strengthen the tax } \\
\text { base. }\end{array}$ & $\begin{array}{l}\text { Submit the draft individual and } \\
\text { corporate income taxes bills to the } \\
\text { National Assembly. }\end{array}$ & $\begin{array}{l}\text { End-December } \\
2007\end{array}$ & \\
\hline
\end{tabular}


Table 2. Cape Verde: Structural Assessment Criteria and Benchmarks for 2007-08, concluded ${ }^{1}$

\begin{tabular}{|c|c|c|c|}
\hline Objectives & Conditionality & Timing & Status \\
\hline \multicolumn{4}{|c|}{ Proposed New Measures } \\
\hline \multicolumn{4}{|c|}{ Structural Assessment Criteria } \\
\hline $\begin{array}{l}\text { Improve fiscal } \\
\text { policy execution }\end{array}$ & $\begin{array}{l}\text { Complete a formal mid-year review of revenue and } \\
\text { expenditure developments that allows for taking } \\
\text { corrective actions if necessary. }\end{array}$ & $\begin{array}{l}\text { End-August } \\
2008\end{array}$ & \\
\hline $\begin{array}{l}\text { Reduce fiscal } \\
\text { risks. }\end{array}$ & $\begin{array}{l}\text { Finalize and publish the mechanism for setting } \\
\text { base utility tariffs }\end{array}$ & $\begin{array}{l}\text { End-March } \\
2008\end{array}$ & \\
\hline \multicolumn{4}{|c|}{ Structural Benchmarks } \\
\hline $\begin{array}{l}\text { Reduce fiscal } \\
\text { risks. }\end{array}$ & $\begin{array}{l}\text { Finalize and publish a revised mechanism for } \\
\text { adjusting petroleum prices. }\end{array}$ & $\begin{array}{l}\text { End-March } \\
2008\end{array}$ & \\
\hline $\begin{array}{l}\text { Improve budget } \\
\text { control }\end{array}$ & $\begin{array}{l}\text { Instituting November } 30 \text { as deadline for granting } \\
\text { new spending commitments to reduce end year } \\
\text { payment pressure. }\end{array}$ & $\begin{array}{l}\text { End-October } \\
2007\end{array}$ & \\
\hline $\begin{array}{l}\text { Strengthen } \\
\text { financial } \\
\text { supervision. }\end{array}$ & $\begin{array}{l}\text { Submit to the National Assembly legislation to } \\
\text { establish a Financial Intelligence Unit. }\end{array}$ & $\begin{array}{l}\text { End-March } \\
2008\end{array}$ & \\
\hline $\begin{array}{l}\text { Strengthen } \\
\text { financial } \\
\text { regulation. }\end{array}$ & $\begin{array}{l}\text { Submit to the National Assembly legislation to } \\
\text { criminalize financing of terrorism and facilitate the } \\
\text { combating of financing of terrorism. }\end{array}$ & $\begin{array}{l}\text { End-March } \\
2008\end{array}$ & \\
\hline $\begin{array}{l}\text { Strengthen } \\
\text { financial } \\
\text { regulation. }\end{array}$ & $\begin{array}{l}\text { Submit to the National Assembly legislation to } \\
\text { strengthen the framework for combating money } \\
\text { laundering. }\end{array}$ & $\begin{array}{l}\text { End-March } \\
2008\end{array}$ & \\
\hline
\end{tabular}

\footnotetext{
${ }^{1}$ Measures added in the third program review.
} 


\section{AtTACHMent II-TeChNiCAL Memorandum OF Understanding ${ }^{1}$}

1. This memorandum sets out the understandings between the Cape Verdean authorities and the IMF staff regarding the definition of assessment criteria and indicative targets and reporting requirements under the first annual program supported by the Policy Support Instrument.

\section{Quantitative Assessment Criteria ANd IndiCAtive TARgets}

\section{A. Net Domestic Borrowing Excluding for Clearance of Arrears and Net Late Payments}

\section{Net domestic borrowing excluding for clearance of arrears and net late} payments is defined as the cumulative change since the start of the calendar year of the net credit to the central government from the banking and nonbanking sectors less (1) the cumulative clearance during the calendar year of the stock of arrears as of the end of the previous year and (2) the cumulative payments during the first three months of the calendar year of expenses authorized by the previous year's budget, and plus the expenses accrued during the current year that will be paid during the first three months of the next calendar year as provisioned for in the budget law (late payments or atrasados). The ceiling will be adjusted downward (upward) by the cumulative downward (upward) deviations in external debt service and upward (downward) by the cumulative downward (upward) deviations in nonproject external financial assistance relative to program assumptions. Also, the end-2007 ceiling will be adjusted upward by the downward deviation in revenue from contracted land sales relative to program assumption, and the 2008 ceilings will correspondingly be adjusted downward by the downward deviation in revenue from contracted land sales in 2007 relative to the 2007 program assumption.

3. Net credit to the central government from the banking and nonbanking system is defined as the overall position of the main central government institutions vis-à-vis the banking and nonbanking system - that is, the stock of all outstanding claims on the central government (loans, advances), and all other government debt instruments, such as long-term government securities) held by the central bank, commercial banks, and nonbank institutions, less all deposits held by the central government with the central bank and with commercial banks. The INPS is not included in central government accounts. Net credit to the central government excludes claims on the Trust Fund (TCMFs).

4. Reporting requirements. Data on the implementation of the budget compiled by the Ministry of Finance and Public Administration will be provided on a quarterly basis, to be submitted not later than five weeks after the end of each quarter, including (i) government domestic revenue by category; (ii) external budget support grants; (iii) government

\footnotetext{
${ }^{1}$ Unchanged from Country Report No. 07/223, except for last sentence of paragraph 2 that has been added.
} 
expenditure, including primary current expenditure, domestic and external interest payments, and capital expenditure, including domestically and budget support financed capital expenditure and estimates of externally project financed capital expenditure; (iv) the gross payment and gross accumulation of domestic accounts payable (atrasados); (v) the gross payment and gross accumulation of domestic payments arrears; (vi) external loan receipts and principal payments; (vii) external arrears payments and accumulation; (viii) bank and nonbank financing; (ix) privatization and land sale receipts; and (x) any other revenue, expenditure, or financing not included above.

5. For the purposes of this memorandum, privatization and land proceeds will be understood to mean all monies received by the government from the sale or concessioning of a public company, organization, or facility to a private company or companies, organization(s), or individual(s), as well as any proceeds generated from the sale of government land and the liquidation of a public company, less restructuring costs.

\section{B. Net Domestic Assets of the Central Bank}

6. The ceiling on the cumulative change, from the beginning of calendar-year 2006, in net domestic assets of the BCV constitutes an assessment criterion. Net domestic assets (NDA) of the BCV are defined as reserve money minus net foreign assets of the BCV, evaluated at the current end-of-period exchange rates. The program ceilings for NDA will be adjusted downward (upward) by the cumulative downward (upward) deviations in external debt service and upward (downward) by the cumulative downward (upward) deviations in nonproject external financial assistance relative to program assumptions. For purposes of calculating the adjusters, these flows will be valued at current exchange rates. Reserve money comprises bank reserves and deposits of the monetary institutions and private sector with the central bank, as well as cash in circulation.

7. Reporting requirements. The preliminary monthly balance sheets of the BCV and the consolidated commercial banks will be transmitted on a monthly basis, with a maximum delay of five weeks. The definitive version of the monthly balance sheet of the BCV will be provided as soon as available.

\section{Ceiling on Nonconcessional External Debt Contracted or Guaranteed by the Central Government}

8. Under the program, ceilings on medium- and long-term, as well as on short-term, nonconcessional external debt constitute assessment criteria. The ceiling on medium- and long-term nonconcessional external debt is on a quarterly basis while the one on short-term nonconcessional external debt is on a continuous basis. Nonconcessional external debt is defined as debt contracted or guaranteed by the central government with a grant element of less than 35 percent, calculated using currency-specific commercial interest reference rates (CIRRs) published by the Development Assistance Committee of the Organization for Economic Cooperation and Development (OECD). Debt rescheduling and debt 
reorganization are excluded from the limits on nonconcessional external debt. The limits on new nonconcessional external debt contracted or guaranteed by the central government (excluding borrowing from the Fund) are specified in Table A1 of the Letter of Intent. The definition of short-term nonconcessional external debt excludes normal short-term (less than one year) import-related financing. The Portuguese government's precautionary credit line in support of the exchange rate peg is also excluded from the definition of nonconcessional external debt. The assessment criterion on medium- and long-term nonconcessional external indebtedness applies not only to debt as defined in point No. 9 of the Guidelines on Performance Criteria with Respect to Foreign Debt (Decision No. 12274-(00/85), 8/24/00) but also to commitments contracted or guaranteed for which value has not been received. With respect to the assessment criterion on short-term nonconcessional external indebtedness, the term "debt" has the meaning set forth in point No. 9 of the Guidelines on Performance Criteria with Respect to Foreign Debt (Decision No. 12274-(00/85), 8/24/00).

9. Reporting requirements. The government of Cape Verde will consult with Fund staff before assuming any liabilities in circumstances where they are uncertain whether the instrument in question falls under the assessment criterion. Details of all new external debt (including government guarantees), indicating terms of debt and creditors, will be provided on a quarterly basis within five weeks of the end of each quarter.

\section{Net International Reserves of the Central Bank}

10. The floor on the cumulative change, from the beginning of calendar-year 2006, in net international reserves (NIR) of the BCV constitutes a assessment criterion under the program. The NIR of the BCV are defined as gross international reserves of the BCV net of its external reserve liabilities, calculated at the current exchange rates. Gross reserves of the $\mathrm{BCV}$ are those that are readily available (i.e., liquid and marketable and free of any pledges or encumbrances), controlled by the BCV and held for the purposes of meeting balance of payments needs and intervening in foreign exchange markets. They include gold, holdings of SDRs, the reserve position at the IMF, holdings of foreign exchange and traveler's checks, demand and short-term deposits at foreign banks abroad, fixed-term deposits abroad that can be liquidated without penalty, and any holdings of investment-grade securities. External liabilities of the BCV comprise liabilities to nonresidents contracted by the BCV with an original maturity of less than a year, any net off-balance-sheet position of the BCV (futures, forwards, swaps, or options) with either resident and nonresidents, any arrears on principal and interest to external creditors and suppliers, and purchases from the IMF. The program floors for the NIR will be adjusted upward (downward) by the cumulative downward (upward) deviations in external debt service and downward (upward) by the cumulative downward (upward) deviations in nonproject external financial assistance relative to program assumptions. For purposes of calculating the adjusters, these flows will be valued at current exchange rates. 
11. Reporting requirements. A table on the NIR prepared by the BCV will be transmitted on weekly basis, with a maximum delay of two weeks.

\section{E. Nonaccumulation of New Domestic Payments Arrears}

12. As part of the program, the government will not accumulate any new domestic payments arrears. This will be monitored through the monthly execution of the cash-flow plan and the corresponding release of budget appropriations. For programming purposes, a domestic payment obligation to suppliers is deemed to be in arrears if it has not been paid within the normal grace period of 60 days (30 days for government salaries and debt service) or such other period either specified by the budget law or contractually agreed with the supplier after the verified delivery of the concerned goods and services, unless the amount or the timing of the payment is subject to good faith negotiations between the government and the creditor.

13. Reporting requirements. The Ministry of Finance and Public Administration, through the D.G.T., will submit on a quarterly basis a detailed table of the stock of domestic payments arrears, including the accumulation, payment, rescheduling and write-off of domestic payments arrears during the quarter. The data are to be provided within four weeks after the end of the quarter.

\section{F. Nonaccumulation of External Payments Arrears}

14. As part of the program, the government will not accumulate any new external payments arrears on a continuous basis. This will be monitored through the monthly execution of the cash-flow plan and the corresponding release of budget appropriations.

15. External arrears are defined as total external debt-service obligations of the government that have not been paid by the time they are due, except where agreements between the government and creditors explicitly provide for a grace period after such obligations falling due. External arrears exclude arrears on external debt, pending the conclusion of debt-rescheduling agreements.

16. Reporting requirements. Data on (i) debt-service payments; and (ii) external arrears accumulation and payments will be transmitted on a quarterly basis by the Ministry of Finance and Public Administration, within five weeks of the end of each quarter. In addition, the government will inform the Fund staff immediately of any accumulation of external arrears. 


\section{Structural ASSESSMent CRiteria ${ }^{2}$}

\section{A. Finalize and publish the mechanism for setting base utility tariffs}

17. The condition for finalizing and publishing the mechanism for setting base electricity and water tariffs will be deemed complete when (i) the technical specifications have been agreed upon between Electra and the autonomous Economic Regulatory Authority (ARE); (ii) the details of the base tariff setting mechanism have been published; and (iii) base tariff levels are brought in line with the agreed mechanism.

\section{B. Continuous application of the mechanisms for setting and adjusting electricity, water, and fuel prices}

18. The condition will be deemed met when (i) base utility tariffs are set, and reset with the periodicity, as specified in the agreed base tariff setting mechanism; (ii) between resetting of base tariffs, utility tariffs are adjusted whenever input costs since the last adjustment have changed cumulatively by more than three percent as specified in the published utility tariff adjustment mechanism; and (iii) within one month of each import shipment of petroleum products, retail petroleum product prices are adjusted and brought in line with the specifications in the retail petroleum price adjustment mechanism.

\section{Other Data Requirements for Program-Monitoring Purposes}

19. Data on exports and imports, including volume and prices and compiled by the Director of Customs and the BCV, will be transmitted on a quarterly basis within five weeks after the end of each quarter. A preliminary quarterly balance of payments, compiled by the $\mathrm{BCV}$, will be forwarded within five weeks after the end of each quarter.

\footnotetext{
${ }^{2}$ See Table A2 of the Letter of Intent of November 29, 2007.
} 


\section{INTERNATIONAL MONETARY FUND}

\section{CAPE VERDE}

Third Review Under the Policy Support Instrument—Informational Annex

Prepared by the African Department

(In consultation with other departments)

Approved by David Andrews and Mark Plant

November 29, 2007

- Relations with the Fund. Describes financial and technical assistance by the IMF and provides information on the safeguards assessment and exchange rate system. At end-September 2007, outstanding PRGF loans amounted to SDR 8.6 million.

- $\quad$ Relations with the World Bank. Describes the World Bank Group program and portfolio; and provides a statement of IFC investments.

- Statistics Issues. Describes the availability and quality of macroeconomic statistics; and its usefulness for surveillance purpose. 


\section{CAPE Verde—Fund Relations}

(As of September 30, 2007)

I. Membership Status: Joined: November 20, 1978;

$\underline{\text { Article VIII }}$

II. General Resources Account:

SDR Million

\%Quota

Quota

9.60

100.00

Fund holdings of currency

9.59

99.93

Reserve Position

0.02

0.17

Holdings Exchange Rate

III. SDR Department:

Net cumulative allocation

Holdings

IV. Outstanding Purchases and Loans:

PRGF Arrangements
SDR Million

0.62

0.02

SDR Million

8.64
\%Allocation

100.00

3.95

\%Quota

90.00

V. Latest Financial Arrangements:

Date of Expiration Amount Approved Amount Drawn

Type Arrangement Date (SDR Million)

$\begin{array}{lllll}\text { PRGF } & \text { Apr 10, 2002 } & \text { Jul 31, 2005 } & 8.64 & 8.64 \\ \text { Stand-By } & \text { Feb 20, 1998 } & \text { Mar 15, 2000 } & 2.50 & 0.00\end{array}$

\section{Projected Payments to Fund ${ }^{1 /}$}

(SDR Million; based on existing use of resources and present holdings of SDRs):

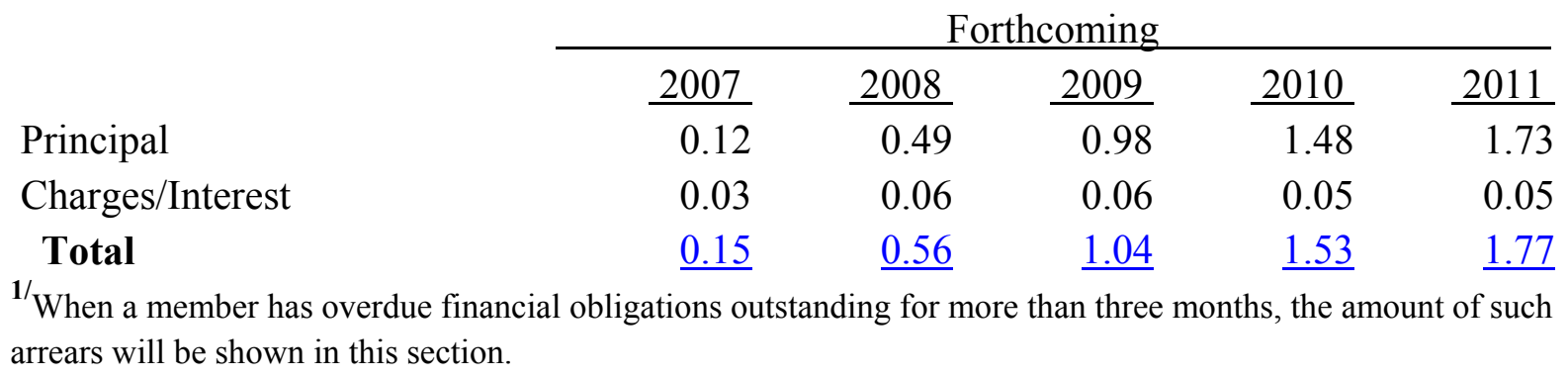

VII. Implementation of HIPC Initiative: Not Applicable

VIII. Implementation of Multilateral Debt Relief Initiative (MDRI): Not Applicable 


\section{Safeguards Assessments}

Under the Fund's safeguards assessment policy, the Bank of Cape Verde (BCV) is subject to a voluntary assessment with respect to the Policy Support Instrument (PSI) approved on July 31,2006 . The necessary documentation has been received from the authorities and the assessment is underway.

Previous safeguards assessment of the BCV was completed in December 2002 with respect to the PRGF arrangement approved on April 10, 2002. The assessment concluded that substantial risks may have existed at the time in the bank's financial reporting framework, its internal audit mechanism, and system of internal controls. While all recommendations but one have been confirmed as implemented by the BCV authorities, the audit opinion of external auditors on the 2005 and 2006 financial statements, and the 2004 management letter indicates that further steps need to be taken.

\section{Exchange Arrangements}

The de facto and de jure exchange rate arrangement of Cape Verde is a conventional fixed peg. The Cape Verde escudo has been pegged to the euro at a rate of CVEsc 110.3 per EUR 1 since January 4, 1999. Cape Verde accepted the obligations under Article VIII of the Articles of Agreement effective July 1, 2004. Cape Verde maintains an exchange system that is free of restrictions on the making of payments and transfers for current international transactions.

\section{Article IV Consultation}

Discussions for the 2006 Article IV consultation and on a medium-term economic program that could be supported by a three-year PSI were held in Praia on April 28-May 16, 2006. The Executive Board concluded the discussions of the 2006 Article IV consultation and approved the request for a PSI on July 31, 2006 (Country Report No. 06/334); the first PSI review on January 19, 2007 (Country Report No. 07/44); the second PSI review on May 7, 2007 (Country Report No. 07/223). www.imf.org.

\section{Technical Assistance}

Since 1985, the Fund has provided technical assistance to the Bank of Cape Verde, the Ministry of Finance, and, more recently, the National Institute of Statistics in several areas:

(i) The Bank of Cape Verde has received technical assistance from MCM in organization and methods, management of external debt, monetary and banking statistics, accounting, credit, and foreign exchange operations, management of public debt, and the separation of the functions of the Bank of Cape Verde, as well as on the choice of exchange rate regime. It has also received technical assistance from STA in monetary and balance of payments statistics. (ii) The Ministry of Finance has received technical assistance from FAD in organization and budgetary procedures, budgeting, tax policy, and tax administration; from STA on fiscal accounting; and from LEG on tax legislation. (iii) The National Institute of Statistics has 
received technical assistance in national accounts and price statistics. Cape Verde is a participant in STA's GDDS Regional Project for Lusophone Africa, and its metadata were posted on the DSBB in February 2004. It is receiving technical assistance to implement the GDDS plan for improvement in the context of this project.

Most recently, technical assistance has been provided in the following areas:

FAD

- June 2004 visit to help the authorities move to a VAT, including the rationalization of the import tariff and the overhaul of the domestic indirect tax system. Many visits and a two-year resident advisor have gone into this effort.

- October 2004, mission to review tax administration, including VAT implementation, and one mission to assist the assessment of tax exemptions and incentives.

- September 2005, mission to assess tax exemptions and incentives.

STA

- National accounts (November 2003 and January-February 2006), balance of payments statistics (February 2004), government finance statistics (March 2004, April 2006, February-March 2007), price statistics (June 2004, May-June 2006, October 2007), and monetary statistics and reporting (March 2007).

\section{MCM}

- Accounting, financial sector regulation, monetary operations and liquidity management (April and May 2004); banking supervision, liquidity management, exchange regime and reserves management (November 2005, March-April 2006, June 2006, November 2006, July 2007).

\section{LEG}

- Tax legislation (several missions from October 2006-November 2007).

- AML/CFT initial assessment (March 2007).

\section{Resident Representative: None.}




\section{CAPE VERde-Relations With The WORLd BANK GROUP}

(November 6, 2007)

\section{Partnership in Cape Verde's development strategy}

1. Cape Verde's first Growth and Poverty Reduction Strategy was prepared in September 2004 and centered on four key pillars for public intervention: (i) promoting good governance; (ii) improving competitiveness and private-sector-led growth; (iii) fostering human capital development; (iv) strengthening social security and solidarity; and (v) improving infrastructure and land use management. The main development challenges for the country revolve around: (i) maintaining macroeconomic stability given external vulnerabilities and uncertainties surrounding resources flows; (ii) achieving further improvements in governance, especially in a decentralized context; (iii) reforming social expenditure programs to reflect the changing nature of demand; and (iv) promoting stronger growth and poverty reduction through improvements in the business climate. The Government's first Annual Progress Report for the PRSP and the Joint IDA-IMF Staff Advisory note (JSAN) were circulated to the Bank's Board on August 10, 2006. The Government is now carrying out an implementation evaluation of the PRSP and launching the preparation of the $2^{\text {nd }}$ GPRSP which is expected to be presented in early 2008 .

\section{World Bank-Fund collaboration in specific areas}

2. The Fund and the Bank continue to collaborate in many areas, including fiscal operations and tax reform; public enterprise reform and privatization; public expenditure management; civil service and pension system reform; utility regulation and private sector development; debt sustainability analyses; and the strengthening of human resource development and the poverty reduction strategy. The Fund and Bank also expect to collaborate on a Financial Sector Assessment Program (FSAP) in 2008 at the Government's request.

\section{World Bank Group strategy}

3. The Bank's FY05-08 Country Assistance Strategy (CAS) was discussed by the Board in February 2005. It is fully aligned with Cape Verde's Growth and Poverty Reduction Strategy (GPRS), as the PRSP is called, and supports efforts in the areas of (i) macroeconomic stability and sound finance and budget systems; (ii) private-sector led growth through an enhanced investment climate and increased competitiveness through public-private partnerships in infrastructure; and (iii) social programs aimed at alleviating poverty and inequity. The Bank is preparing a new CAS which would be based on the $2^{\text {nd }}$ GPRSP and is expected to be presented at the Bank's Board in May 2008. 


\section{World Bank Group activities and assessment of country policies}

\section{IDA portfolio}

4. Under the CAS, the Bank is supporting the implementation of the GPRSP and has made a significant shift toward programmatic lending, (60\% of CAS commitments) to support policy reforms that complement investment operations. Programmatic lending has been an important instrument to promote the harmonization agenda, and through budget support MOUs with Government and donors the Bank Group has helped catalyzed additional donor participation and build partnerships. The Cape Verde portfolio is one of the best performing in the Africa region. All projects in the IDA portfolio are rated satisfactory or higher in terms of achieving project development objectives.

5. With regard to programmatic lending, the Bank has prepared three Poverty Reduction Support Credits under the current CAS. The first PRSC for US\$15 million equivalent was approved in 2005, a second PRSC for US\$10 million equivalent was approved by the Board in June 2006, and a third PRSC for \$10 million was approved in March 2007. All three PRSCs focused on: (i) promoting good governance, reinforce effectiveness and guarantee equity; (ii) developing and upgrading human capital; and (iii) improving the effectiveness and sustainability of the social protection system. The Bank is preparing a fourth PRSC in the amount of US\$6 million equivalent to be presented to the Board in 2008.

6. With regard to investment lending, there are three IDA operations supporting: 1) growth and competitiveness; 2) the implementation of the national HIV/AIDS strategy; and 3) a road sector support program. A GEF grant is supporting investments in wind mill power.

7. In terms of strengthening the country's physical infrastructure and competitiveness, IDA's assistance is as follows:

The Road Sector Support Project aims to support critical road transport infrastructure needs through a combination of the following approaches: (i) improving access roads linking villages and rural areas to main roads; (ii) filling critical gaps to establish a minimum continuous network within an island with the construction of a short section of new road (8$10 \mathrm{~km}$. on St. Nicolau Island); and (iii) filling a major gap in an island network, through the construction of bridges or providing access to a chronically poor access zone.

An IDA financed Energy and Water Project closed in June 2007 and had supported efforts to improve the supply of power, water and sanitation systems, and to increase the efficiency of the power and water sectors, to lower the barriers to development of renewable energy sources and to foster sound management of water resources. A GEF Energy and Water Project, which was linked to the IDA operation, continues to support the development of renewable energy sources and is supporting investments in wind mill parks. 
The Growth and Competitiveness Project aims to broaden the base of private participation in Cape Verde's economic growth, enhance private sector competitiveness and further develop its financial sector. This would be achieved through a series of actions supported by the project, notably: (a) financial sector reform, including pension reform; (b) investment climate reform, which includes, but is not limited to: (i) tax reform, (ii) alleviation of administrative barriers, (iii) improvement of supply chains, and (iv) legal reform; (c) post privatization and divestiture reforms; and (d) private sector and institutional capacity building. The project thus helps to sustain the Government's poverty alleviation efforts by generating increased employment opportunities through improved international competitiveness.

8. In the social sectors, the HIV/AIDS Project supports (i) mitigation of the health and socioeconomic impact of HIV/AIDS at the individual, household, and community levels, thus sustaining an economically productive population, and (ii) establishment of a strong and sustainable national capacity to respond to the epidemic.

\section{Assessment of country policies}

9. The authorities of Cape Verde and development partners, including the Bank, have undertaken a substantial body of analytic work to assess key social, structural, and sectoral development policies and identify policy and institutional reform priorities to support growth and poverty reduction. These efforts resulted, inter alia, in the adoption in April 2003 of a Statement of development policies for private sector development, which provided the basic framework for the preparation of the Growth and Competitiveness project.

10. In FY07, advisory services funded by the World Bank have in large part been related to infrastructure, fisheries and economic management (public service reform). Proposed economic and sector work (ESW) includes core diagnostics, annual public expenditures reviews (PER), and general economic work to sustain macroeconomic and sectoral dialogue. The following studies have also been part of the current CAS: Investment Climate Assessment (FY06), Integrated CFAA/CPAR (FY06); Rural Development Assessment (FY07), Long term Growth and Competitiveness Study (FY08), and a Poverty Assessment Update (FY08).

11. As of November 2007, the IDA portfolio represents commitments of US\$44 million equivalent and an undisbursed balance of about US\$14 million equivalent. The involvement of the International Finance Corporation (IFC) in Cape Verde remains limited (see tables below). 


\section{Statement of IDA Operations}

(As of November 6, 2007; in millions of U.S. dollars)

\begin{tabular}{|c|c|c|c|c|c|}
\hline $\begin{array}{c}\text { Credit } \\
\text { No. }\end{array}$ & Projects & $\begin{array}{c}\text { Principal } \\
\text { Amount }\end{array}$ & Undisbursed & $\begin{array}{c}\text { Approved } \\
\text { Date }\end{array}$ & $\begin{array}{c}\text { Closing } \\
\text { Date }\end{array}$ \\
\hline 32170 & Roads Sector Support & 15.00 & 4.3 & 19-May-05 & 31-Aug-10 \\
\hline 37550 & Growth \& Competitiveness & 14.50 & 5.1 & 13-May-03 & 28-Feb-08 \\
\hline 36290 & HIV/AIDS & 14.00 & 3.7 & 28-Mar-02 & 31-Dec-08 \\
\hline & Total active projects & 43.50 & 13.1 & & \\
\hline
\end{tabular}

Note: Disbursed amount may be higher than commitment (approved amount) due to exchange rate vis-à-vis SDR.

\section{Statement of IFC Investments}

(In millions of U.S. dollars)

\begin{tabular}{|c|c|c|c|c|c|c|c|c|c|c|}
\hline \multirow[b]{2}{*}{$\begin{array}{c}\text { FY } \\
\text { Approv. }\end{array}$} & \multirow[b]{2}{*}{ Company } & \multirow[b]{2}{*}{$\begin{array}{l}\text { Type of } \\
\text { Business }\end{array}$} & \multicolumn{4}{|c|}{ Original Gross Commitments } & \multicolumn{4}{|c|}{ Disbursed } \\
\hline & & & Loan & Equity & Quasi & Partic & Loan & Equity & Quasi & Partic. \\
\hline 1992 & Growela 1/ & $\begin{array}{l}\text { Shoe manu- } \\
\text { facturing }\end{array}$ & 0.15 & 0.00 & 0.00 & 0.00 & 0.15 & 0.00 & 0.00 & 0.00 \\
\hline 2004 & CECV 2/ & $\begin{array}{l}\text { Finance and } \\
\text { insurances }\end{array}$ & 4.70 & 0.00 & 0.00 & 0.00 & 4.70 & 0.00 & 0.00 & 0.00 \\
\hline \multicolumn{3}{|c|}{ Total portfolio } & 4.85 & 0.00 & 0.00 & 0.00 & 4.85 & 0.00 & 0.00 & 0.00 \\
\hline
\end{tabular}

$1 /$ Growela $=$ shoe manufacturing.

2/ CECV = Caixa Econômica de Cabo Verde. 


\section{CAPE VERDE-STATISTICS ISSUES}

20. The quality of economic and financial data is generally adequate for surveillance and program monitoring, although there is a need for substantial improvements in some areas. Weaknesses in the fiscal data hamper the monitoring of some aspects of fiscal performance. Weaknesses include large statistical discrepancies, non-compliance with best accounting practices, and shortcomings in the institutional coverage and sectorization. Other shortcomings of particular concern are weaknesses in the national accounts, a lack of shortterm activity indicators besides the confidence barometer, and gaps in the tracking of large external flows — notably FDI, emigrant deposits, and remittances.

21. The authorities are taking steps to strengthen statistics, but the statistical system still suffers from a shortage of financial and human resources. A comprehensive master plan has been developed under the direction of the National Statistical Institute (INE), which assesses the need for upgrading the various agencies that constitute the statistical system and outlines the steps needed for broadening and improving all areas of statistics. The plan envisages data improvements in national accounts (annual and quarterly), business statistics (including a new business census), demographic and social statistics (including a household survey to update the poverty profile), trade statistics (retail and international trade) macroeconomic indicators; labor market statistics and sectoral statistics, and the regular update of the CPI basket (every half a decade). Cape Verde's development partners have already committed a substantial part of the estimated US\$15 million required to implement the plan over 2006-10.

22. The country is a participant in the Fund's General Data Dissemination System (GDDS) since February 2004 and has benefited from STA technical assistance (STA TA) under the GDDS project for Lusophone Africa. The main purpose of this project was to assist the government in implementing the GDDS plans for improvement, in particular regarding the adoption of the Government Finance Statistics Manual 2001 (GFSM 2001). In this context, a GFS mission visited the country in March 2004, and there was a follow-up mission in April 2006 and March 2007. STA TA has also been provided in other areas, such as national accounts, consumer prices (with a recent STA TA mission in May-June 2006, and October 2007 in preparation for the launching of the new CPI in November 2007), monetary and financial statistics, and balance of payments statistics.

\section{Real sector}

23. Although some improvements have been made to the national accounts, significant weaknesses remain. The timeliness of the data has improved, with the National Statistical Institute (INE) releasing production and expenditure based GDP data for 2002-03 in 2005 and providing production based GDP estimates for 2004-06 to the mission in October 2007. However, the lag is still large when compared to international standards. The base year for the constant price estimates, moreover, is outdated. 
24. INE is currently working on a complete overhaul of the national accounts. The overhaul includes updating the national accounts benchmark and base years from 1980 to 2002, and adopting the 1993 SNA. The ultimate objective is the timely compilation of GDP by industry and expenditure categories at current and constant prices as well as the institutional sector accounts.

25. A full implementation of the $1993 S N A$ would require a substantial improvement in the source data collection programs for which sufficient capacity is currently lacking. The 2006 STA mission found that there is a critical need for improving the timeliness and accuracy of the national accounts source data, in particular the business survey. The capacity of INE staff working on national accounts was found to be overstretched, assessing in detail and correcting individual source data entries - a task which in most countries is not undertaken by the national accounts compilers. The statistical masterplan will address many of these challenges.

26. A new CPI is expected to be released in November 2007. The current official CPI is based on weights dating back to 1989 , four years before the liberalization of imports, which changed considerably the consumption pattern. INE has worked with the assistance of the National Statistics Institute of Portugal, and benefited from a STA CPI mission in May-June, 2006, October 2006, and October 2007 aimed at supporting the introduction and dissemination of the revised CPI. The new index will benefit from new weights, an updated commodity basket, and the use of new compilation software.

\section{Government finance}

27. Progress has been made to improve fiscal data. Benefiting from TA support under the GDDS project for Lusophone Africa, the Government Finance Statistics (GFS) compilation system is being upgraded. Most deposits previously held with commercial banks have been consolidated at the central bank. SIGOF, the Integrated Online Budget Management System, will be expanded to include all semi-autonomous institutes and most municipalities by the end of 2007. In addition, a new chart of government accounts (PNCP) will be implemented in 2008 adopting accrual accounting and double-entry principle.

28. Serious quality concerns remain. The fiscal accounts are subject to large statistical discrepancies, flows and stocks are not consistent, and recording of arrear accumulation and clearance operations is not in line with best practices. Significant delay in donor reporting of project-financing also affects the accuracy of fiscal data. Despite the recent revision of external debt data, significant weaknesses affect the preparation of debt sustainability analyses. Multilateral debt statistics regularly differ from data received by creditors, and debt service projections cannot be reconciled with the debt stock. 


\section{Money and banking}

29. The quality of the monetary and financial statistics is adequate, both in terms of accuracy and timeliness. A statistics mission undertaken in March 2007 assisted BCV in finalizing the standardized report forms (SRFs) for reporting monetary statistics to STA. SRF-based monetary data have been published in the IFS Supplement since June 2007. These data are fully aligned with the recommendations of the Monetary and Financial Statistics Manual. The mission also started developing an integrated monetary database to meet STA, AFR, and BCV statistical needs. Currently, there are gaps in tracking the source and direction of changes in emigrant deposits, which cause difficulties for gauging the appropriateness of the monetary policy stance.

\section{Balance of payments}

30. With technical assistance from STA, accuracy, periodicity, and timeliness of balance of payments statistics compiled by BCV have continued to improve. A greater use of surveys, combined with the International Transactions Reporting System implemented by the $\mathrm{BCV}$, has permitted a significant expansion of data sources and statistical coverage, which, to a large extent, follow the recommendations of the $5^{\text {th }}$ edition of the Balance of Payments Manual. Dissemination of quarterly BOP data on the BCV website has been regular. 


\section{Cape Verde: Common Indicators Required for Surveillance}

(As of November 6, 2007)

\begin{tabular}{|l|c|c|c|c|c|}
\hline & $\begin{array}{c}\text { Date of } \\
\text { latest } \\
\text { observation }\end{array}$ & $\begin{array}{c}\text { Date } \\
\text { received }\end{array}$ & $\begin{array}{c}\text { Frequency } \\
\text { of Data }^{6}\end{array}$ & $\begin{array}{c}\text { Frequency } \\
\text { of } \\
\text { Reporting }^{6}\end{array}$ & $\begin{array}{c}\text { Frequency } \\
\text { of } \\
\text { publication }^{6}\end{array}$ \\
\hline Exchange Rates & $11 / 06 / 07$ & $11 / 06 / 07$ & $\mathrm{D}$ & $\mathrm{D}$ & $\mathrm{D}$ \\
\hline $\begin{array}{l}\text { International Reserve Assets and } \\
\text { Reserve Liabilities of the } \\
\text { Monetary Authorities }\end{array}$ & Sept-07 & Oct-07 & $\mathrm{M}$ & $\mathrm{M}$ & $\mathrm{M}$ \\
\hline Reserve/Base Money & Sept-07 & Oct-07 & $\mathrm{M}$ & $\mathrm{M}$ & $\mathrm{M}$ \\
\hline Broad Money & Sept-07 & Oct-07 & $\mathrm{M}$ & $\mathrm{M}$ & $\mathrm{M}$ \\
\hline Central Bank Balance Sheet & Sept-07 & Oct-07 & $\mathrm{M}$ & $\mathrm{M}$ & $\mathrm{M}$ \\
\hline $\begin{array}{l}\text { Consolidated Balance Sheet of } \\
\text { the Banking System }\end{array}$ & Sept-07 & Oct-07 & $\mathrm{M}$ & $\mathrm{M}$ & $\mathrm{M}$ \\
\hline Interest Rates & Sept-07 & Oct-07 & $\mathrm{M}$ & $\mathrm{M}$ & $\mathrm{M}$ \\
\hline Consumer Price Index & Sept-07 & Oct-07 & $\mathrm{M}$ & $\mathrm{M}$ & $\mathrm{M}$ \\
\hline $\begin{array}{l}\text { Revenue, Expenditure, Balance } \\
\text { and Composition of Financing } \\
\text { General Government }\end{array}$ & June-07 & Ago-07 & $\mathrm{Q}$ & $\mathrm{Q}$ & $\mathrm{Q}$ \\
\hline $\begin{array}{l}\text { Revenue, Expenditure, Balance } \\
\text { and Composition of Financing } \\
\text { Central Government }\end{array}$ & Jun-07 & Ago-07 & $\mathrm{Q}$ & $\mathrm{Q}$ & $\mathrm{Q}$ \\
\hline $\begin{array}{l}\text { Stocks of Central Government } \\
\text { and Central Government- } \\
\text { Guaranteed Debt }\end{array}$ & Jun-07 & Oct-07 & $\mathrm{Q}$ & $\mathrm{Q}$ & $\mathrm{Q}$ \\
\hline $\begin{array}{l}\text { External Current Account } \\
\text { Balance }\end{array}$ & Sept-07 & Oct-07 & $\mathrm{Q}$ & $\mathrm{Q}$ & $\mathrm{Q}$ \\
\hline $\begin{array}{l}\text { Exports and Imports of Goods } \\
\text { and Services }\end{array}$ & Sept-07 & Oct-07 & $\mathrm{Q}$ & $\mathrm{Q}$ & $\mathrm{Q}$ \\
\hline GDP/GNP & 2006 & Oct-07 & $\mathrm{A}$ & $\mathrm{A}$ & $\mathrm{A}$ \\
\hline Gross External Debt & Oct-07 & $\mathrm{Q}$ & $\mathrm{Q}$ & $\mathrm{Q}$ \\
\hline
\end{tabular}

${ }^{1}$ Includes reserve assets pledged or otherwise encumbered as well as net derivative positions.

${ }^{2}$ Both market-based and officially-determined, including discount rates, money market rates, rates on treasury bills, notes and bonds.

${ }^{3}$ Foreign, domestic bank, and domestic nonbank financing.

${ }^{4}$ The general government consists of the central government (budgetary funds, extra budgetary funds, and social security funds) and state and local governments.

${ }^{5}$ Including currency and maturity composition.

${ }^{6}$ Daily $(D)$, weekly $(W)$, monthly $(M)$, quarterly $(Q)$, annually $(A)$, irregular (I); and not available (NA). 


\title{
INTERNATIONAL DEVELOPMENT ASSOCIATION
}

\author{
CAPE VERDE
}

\section{Joint Fund-Bank Debt Sustainability Analysis}

Prepared by the staffs of the International Development Association and the International Monetary Fund

Approved by Sudhir Shetty and Brian Pinto (World Bank) and David Andrews and Mark Plant (IMF)

November 29, 2007

The risk of debt distress in Cape Verde remains low. Domestic debt is expected to continue to decline owing to a prudent fiscal stance in line with the authorities' commitment with the Fund and dialogue with World Bank. Moreover, all external debt indicators remain below the relevant thresholds under the baseline and alternative scenarios, even after concessional external financing declines as the country graduates from the United Nations' leastdeveloped-country (LDC) status in 2008. Continued flow of foreign direct investment is projected to finance a large part of the external current account deficit.

\section{BACKGROUND ${ }^{13}$}

\section{This DSA updates the DSA done in 2006 at the inception of the Policy Support} Instrument (PSI) of the IMF. ${ }^{14}$ This is the second DSA for Cape Verde that applies the methodology of Fund-World Bank debt sustainability framework (DSF) for low income countries. ${ }^{15}$ In accordance with the framework, this DSA compares the expected trajectory of debt indicators with the thresholds for countries with strong policies and institutions (Table 4) ${ }^{16}$ Based on 2006 debt estimates updated during the mission for the third review of the PSI in October 2007, the analysis maintains the previous assessment that the risk of debt distress in Cape Verde remains low.

\footnotetext{
${ }^{13}$ This analysis includes only central government debt and guarantees, thus excluding municipalities and stateowned enterprises.

${ }^{14}$ Appendix II of IMF Country Report No. 06/334, 09/2005.

${ }^{15}$ Although Cape Verde is not a low-income country and is not eligible for HIPC assistance and MDRI, it is eligible for PRGF and IDA financing based on the small island exception. In July 2007 Cape Verde became eligible to (limited) IBRD lending in addition to IDA, thus becoming a blend country. After graduation from LDC status in 2008, it will continue to be a blend country.

${ }^{16}$ Cape Verde's average score under the World Bank's Country Policy and Institutional Assessment (CPIA) in 2004-6 is 4.1, above the floor for strong performers of 3.75 .
} 


\section{In 2006, the total central government net debt and guarantees were reduced} markedly as a percentage of GDP. Although the guarantees increased, the total nominal debt fell to 77 percent of GDP in 2006, down from 87 percent in 2005. Rapid GDP growth in 2006 and prudent fiscal policy led to this reduction (Table 8). The central government external debt was reduced from 54 percent of GDP to 48 percent during 2006 while its domestic debt was reduced from 33 percent of GDP to 29 percent.

33. Multilateral and bilateral agencies continue to be the largest external creditors. Commercial creditors account for less than 2 percent of the external debt. The World Bank (IDA) was the largest multilateral creditor, followed by the African Development Fund. Portugal is the main bilateral lender (Table 1).

Table 1. Cape Verde: Central government external debt, 2004-06

\begin{tabular}{|c|c|c|c|}
\hline & 2004 & 2005 & 2006 \\
\hline & \multicolumn{2}{|c|}{ Actual } & Est. \\
\hline & \multicolumn{3}{|c|}{ (In percent of the nominal debt) } \\
\hline Total & 100 & 100 & 100 \\
\hline Multilaterals & 77 & 78 & 78 \\
\hline IMF & 2 & 2 & 2 \\
\hline IDA & 40 & 42 & 44 \\
\hline Others & 35 & 33 & 32 \\
\hline Official Bilaterals & 19 & 19 & 19 \\
\hline Paris Club members & 12 & 13 & 12 \\
\hline Non-Paris Club members & 4 & 5 & 7 \\
\hline Commercial & 4 & 3 & 2 \\
\hline \multicolumn{4}{|c|}{ Memorandum: Total nominal external debt of the central government } \\
\hline In US\$ million ${ }^{1}$ & 553 & 513 & 598 \\
\hline In percent of GDP & 55 & 54 & 48 \\
\hline
\end{tabular}

\section{MEDIUM-TERM MACROECONOMIC FRAMEWORK AND ASSUMPTIONS OF THE DSA}

34. In the baseline macroeconomic framework of this revised DSA, economic growth is projected to be slightly higher than assumed in the previous DSA (Table 2). Real GDP growth is expected to average about 7 percent through 2012 underpinned by continued robust growth in the services and construction sectors driven by tourism. Tourism arrivals have increased and several large projects in tourism entered the pipeline since the last DSA. The government's strategy involves promoting tourism by expanding infrastructure, improving the business environment. As result, the investment promotion agency has signed investment protocols with several private hotel operators. This gives reasonable confidence that many of these investments will indeed take place. As the initial phase of the tourism boom tapers off, GDP growth is conservatively assumed to slow gradually to 5 percent over the long term, below historical levels. However, the authorities are of the view that the growth rates driven by the tourism boom will be stronger and last longer than this DSA's assumptions. 
Consistent with the peg to the Euro, annual inflation is expected to stay between 2 and 3 percent in line with that of Cape Verde's main trading partners.

\section{Strong FDI inflows are assumed to finance the higher current account deficit}

and limit the need for new external borrowing. The increase in the current account deficit reflects the growth of imports for capital formation and tourism (Table 2). Additionally, during the execution of the Millennium Challenge Corporation (MCC) compact, imports will be temporarily above the trend. FDI is assumed to remain above 10 percent of GDP until 2015 and to gradually decline after the investment boom fades. Tourism exports will continue to rise faster than GDP throughout the projection period, evidencing the specialization of Cape Verde in tourism. Tourism growth will compensate the increase in imports and the decline in remittances. It is assumed that private current transfers, including emigrant remittances, will decline from 20 percent of GDP in 2006 to 12 percent in 2027 as incomes in Cape Verde grow faster than in emigrant recipient countries and emigration recedes.

\section{Prudent fiscal policy is expected to continue in line with the PSI objectives,} which will help preserve the low risk of debt distress. In the baseline scenario, revenue growth and expenditure restraint will maintain the primary fiscal deficit below the debtstabilizing deficit throughout the projection period (Table 8). Tax revenues are expected to be buoyant underpinned by both robust economic growth and improvements in tax administration, as well as rationalization of tax exemptions and broadening of the tax base, which will compensate for the expected decline in grants. Improvements in budget execution and monitoring, including under the IMF's PSI, are assumed to induce further restraint on current expenditures. Capital expenditures are assumed to remain above 10 percent of GDP throughout the projection period, in line with the authorities' intentions to improve the country's infrastructure (Table 3 ).

Table 2. Cape Verde: Macroeconomic Baseline Assumptions, 2007-27

(in percent, unless otherwise indicated)

\begin{tabular}{|c|c|c|c|c|c|}
\hline & \multirow{2}{*}{$\begin{array}{c}\text { Average (1996- } \\
2006) \\
\end{array}$} & \multicolumn{2}{|c|}{5 years ahead } & \multicolumn{2}{|c|}{ 6-20 years ahead } \\
\hline & & Previous DSA & Current DSA & Previous DSA & Current DSA \\
\hline Real GDP growth & 6.8 & 6.2 & 7.3 & 5.1 & 5.2 \\
\hline Inflation & 2.6 & 2.2 & 2.3 & 2.1 & 2.0 \\
\hline Exports of G\&S growth (in US dollars) & 14.8 & 6.9 & 15.2 & 8.5 & 9.7 \\
\hline Imports of G\&S growth (in US dollars) & 8.9 & 9.0 & 14.0 & 6.0 & 8.4 \\
\hline Private transfers, incl. remittances (percent of GDP) & 19.4 & 20.5 & 17.3 & 11.5 & 13.7 \\
\hline Current account balance (percent of GDP) & -9.3 & -9.7 & -13.2 & -7.5 & -12.0 \\
\hline Foreign direct investment (percent of GDP) & 3.5 & 3.2 & 12.2 & 2.0 & 9.3 \\
\hline Current acct. and FDI (Percent of GDP) & -5.8 & -6.5 & -1.0 & -5.5 & -2.6 \\
\hline Grant element of new external borrowing & $\ldots$ & 28.3 & 27.9 & 9.3 & 9.5 \\
\hline Exchange rate, nominal currency per US dollar, p.a & 101.3 & 92.7 & 79.4 & 92.1 & 78.7 \\
\hline Public sector revenue and grants (percent of GDP) & 30.3 & 34.1 & 29.2 & 28.8 & 28.0 \\
\hline
\end{tabular}

Source: Cape Verdian authorities, staff estimates and projections.

\section{Grants and external financing assumptions under this revised DSA are conservative, in line with Cape Verde's impending graduation from LDC status.}


Assumptions on concessional financing and grants have been significantly revised downwards compared to the previous DSA. It is now assumed that as Cape Verde graduates from LDC status in 2008, grants as a percentage of GDP and the degree of concessionality of the new borrowing will decline (Table 6 and 8, Figure 1). The share of commercial financing in total new external borrowing is assumed to gradually increase to 85 percent until 2027 , and the grant element of new external borrowing will decline to zero. Because commercial loans have shorter maturity, the debt service will also increase (Table 7, Figure 1, bottom charts). The lower concessionality along with an assumed decrease in current transfers will contribute to the widening of the current account deficit. The interest rate on the domestic debt remains constant at 4.3 percent throughout the period, and the marginal borrowing in the stress scenarios is assumed to be on commercial terms (Table 7).

Table 3. Cape Verde: Comparison of Scenarios, 2008-12

\begin{tabular}{lcr}
\hline & \multicolumn{2}{c}{ Scenario } \\
\cline { 2 - 3 } & Baseline & Scaling-up \\
\hline Real GDP growth, 2007-2027 & (Percent of GDP, period average) \\
Imports of G\&S (in US\$) & 5.8 & 5.8 \\
Capital expenditures & 78.8 & 72.4 \\
Fiscal balance & 12.6 & 14.5 \\
External current account balance & -3.2 & -5.2 \\
\hline
\end{tabular}

\section{EXternal Central Government DebT Sustainability}

\section{A. Baseline scenario}

38. Cape Verde's disciplined macroeconomic policy framework, continued strong FDI flows, and the resulting low reliance on external central government debt will maintain low risk of debt distress. The ratio of NPV of the external debt-to-GDP is expected to decline during most of the period because the external borrowing-to-GDP ratio generally stays below the debt-stabilizing ratio (Tables 4, 6 and 7, Figure 1). The external borrowing is small because FDI finances a large part of the higher current account deficit (Tables 2 and 6). Therefore, the NPV of the central government external debt declines from 26 percent of GDP in 2007 to below 22 percent of GDP after 2009, comfortably below the debt-burden threshold. The ratios of debt-to-exports and to revenues also remain below the indicative thresholds. Because commercial loans have shorter maturities, debt service ratios increase. Nevertheless, the debt service indicators remain below the thresholds, suggesting that roll-over difficulties are unlikely (Table 7 and Figure 1). The assessment of low risk hinges on the assumption that tourism exports and FDI flows are sufficient to finance the imports used as inputs in the tourism industry. This assumption is reasonable because tourism exports are partly produced with domestic factors. 


\section{B. Alternative scenarios and stress tests}

39. The government's external debt indicators remain well below the thresholds in all scenarios. The most extreme stress scenario is a hypothetical 30-percent devaluation of the exchange rate in 2008. It causes a one-off increase of 7 percent of GDP in the NPV of the debt. However, even in this stress scenario the external debt ratios remain below the threshold as the NPV of the external debt before the shock is low.

Table 4. Cape Verde: Central Government External Debt Ratios

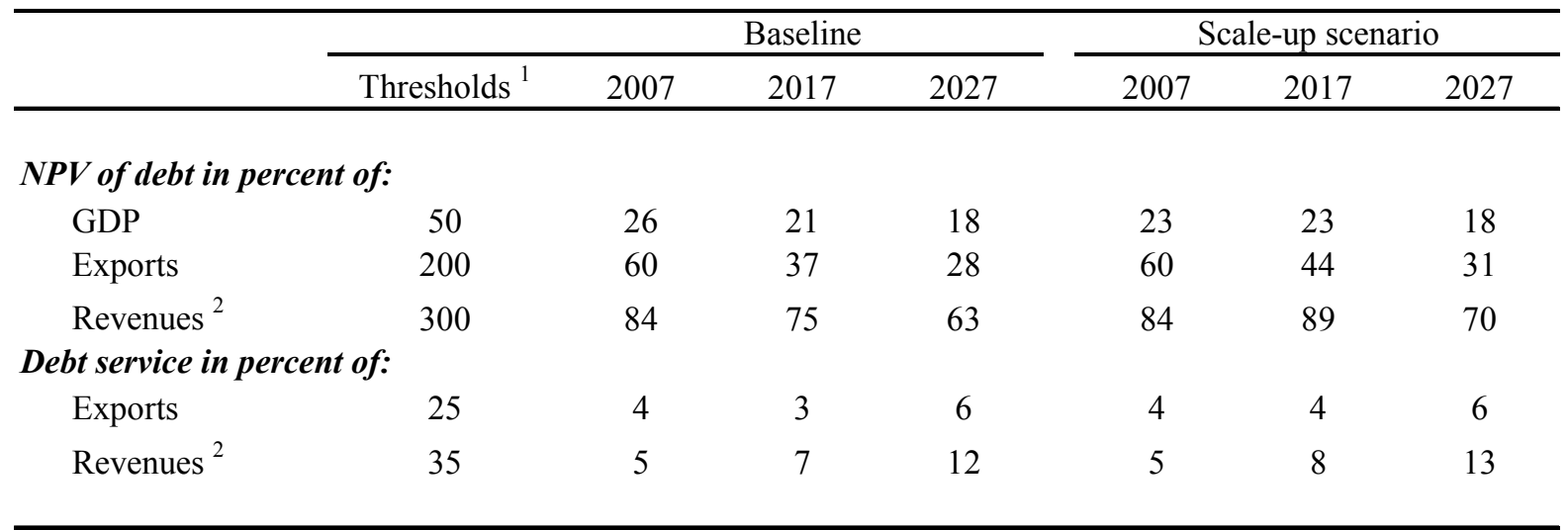

Source: Ministry of Finance; and staff extimates.

${ }^{1}$ Based on Cape Verde's 2004-06 classification as a strong performer.

2 Including grants.

\section{Scaling-up scenario}

40. An alternative scenario of scaling-up borrowing under commercial terms and capital expenditures has been simulated (Table 3). Although the authorities have not yet finalized their medium-term investment program, they intend to explore new mechanisms to scale-up infrastructure investment. To show the robustness of the debt sustainability under the scaling-up scenario, this alternative scenario assumes that Cape Verde will borrow US\$ 35 million on commercial terms in each of the five years covering 2008-12 in addition to the borrowing in the baseline scenario. ${ }^{17}$ This extra borrowing is used to scale-up public capital expenditure and is matched by a corresponding increase in imports. This scenario simulates, for example, additional commercial borrowing to invest in the electricity sector in 2008 and the envisaged borrowing from the IBRD. The additional interest expense arising from the scaling up of external borrowing is financed with domestic borrowing. For prudence, economic growth is maintained at the same level of the baseline scenario.

41. The risk of debt distress also remains low in the scaling-up scenario. All ratios remain well below the thresholds throughout the forecast horizon. The additional

${ }^{17}$ This value corresponds to the ceiling for non-concessional external borrowing agreed under the PSI. 
US\$35 million borrowed in this scenario, equivalent to 2 percent of GDP on average during 2008-12, does not represent an important burden given that the debt is low and that GDP growth rate is still higher than the debt accumulation rate. Further, the debt ratios remain below threshold even if shocks are simulated to hit on top of the scaling up scenario.

\section{Total Central Government Debt Sustainability}

\section{A. Baseline scenario}

42. In line with the PSI objectives, the total central government debt and guarantees remain below current level throughout the forecast horizon in the baseline scenario. This reflects the Bank and Fund staff's expectation that the good fiscal policy performance will be maintained in the long term. In addition to the expected decline in the external debt ratio discussed previously, the domestic central government debt ratio is reduced to below 20 percent of GDP by 2008, one year ahead of the PSI target (Table 8). The low fiscal deficits and the contribution of the GDP growth to the debt ratio are the main reason for the decline. After 2013, the total central government debt-to-GDP ratio stabilizes around 34 percent of GDP (Figure 2). The ratios of debt-to-revenue and debt service-to-revenue also suggest that roll-over difficulties are unlikely to arise.

\section{B. Alternative scenarios and stress tests}

43. In the long term, endogenous risks to the sustainability of the total central government debt are more threatening than exogenous shocks. The current policies are consistent with maintaining a low risk of debt distress, which is evidenced by the fact that the debt ratios remain at low levels in the scenario of unchanged balance from the 2007 level (Table 9, and Figure 2). However, there is the endogenous risk that prudent fiscal policies will not be maintained in the long term. In this scenario, the primary deficit returns to the historical levels, which is substantially higher than the debt-stabilizing level. Therefore, in this scenario the debt rises sharply (scenario A1 of Table 9, Figure 2). ${ }^{18}$

\section{An exogenous shock alone does not increase the risk of debt distress to a} significant level. The most extreme test is one in which an exogenous shock temporarily widens the primary deficit to 11 percent of GDP $^{19}$ during 2008-09 (scenario B2 in Table 9,

\footnotetext{
${ }^{18}$ This historical scenario assumes also that GDP growth is at historical average of 7 percent, more optimistic than baseline projections.

${ }^{19}$ Equivalent to historical balance minus one standard deviation.
} 
Table 5. Total Central Government Debt Ratios, 2007-27

\begin{tabular}{|c|c|c|c|c|c|c|}
\hline & \multicolumn{3}{|c|}{ Baseline } & \multicolumn{3}{|c|}{ Scale-up scenario } \\
\hline & 2007 & 2017 & 2027 & 2007 & 2017 & 2027 \\
\hline \multicolumn{7}{|c|}{ NPV of total debt in percent of: } \\
\hline GDP & 47 & 33 & 34 & 47 & 41 & 40 \\
\hline $\mathrm{o} / \mathrm{w}$ domestic debt & 23 & 14 & 17 & 23 & 17 & 21 \\
\hline Revenues $^{1}$ & 152 & 119 & 120 & 152 & 146 & 143 \\
\hline \multicolumn{7}{|l|}{ Debt service in percent of: } \\
\hline Revenues $^{1}$ & 11 & 11 & 18 & 11 & 14 & 20 \\
\hline
\end{tabular}

Figure 2). Because in this scenario the debt ratios increase only temporarily, the low risk of debt distress is preserved throughout the period. This conclusion applies also to the situation where the extreme shock hits on top of the scale up scenario.

\section{Scaling-up scenario}

45. Under the scaling up of borrowing and public investment, the low risk of debt distress is preserved. The fiscal deficit during the 5 years of the scaling up is smaller than the debt-stabilizing deficit, causing the domestic debt to remain around the PSI target of 20 percent of GDP during the projection period (Table 5). All the other total central government debt indicators remain within reasonable levels in the scaling up scenario, even if shocks are simulated to hit on top of the scaling up scenario (Tables 5 and 8, and Figure 2).

\section{CONCLUSION}

\section{The debt sustainability analysis concludes that the risk of debt distress in Cape} Verde is low. This is notwithstanding the conservative assumptions made in the baseline scenario including on financing terms. The conclusion hinges on the assumption that FDI flows and tourism exports will fully offset the growth of imports caused by them. The most important threat to debt distress risk is a return to historical fiscal deficits, which highlights the need for continued fiscal prudence. Based on the expectation that the fiscal prudence will continue during the projection period, the assessment of distress risk is low. The risk of debt distress for Cape Verde remains low also under large exogenous shocks and under a 5-year scaling up of capital investment. 


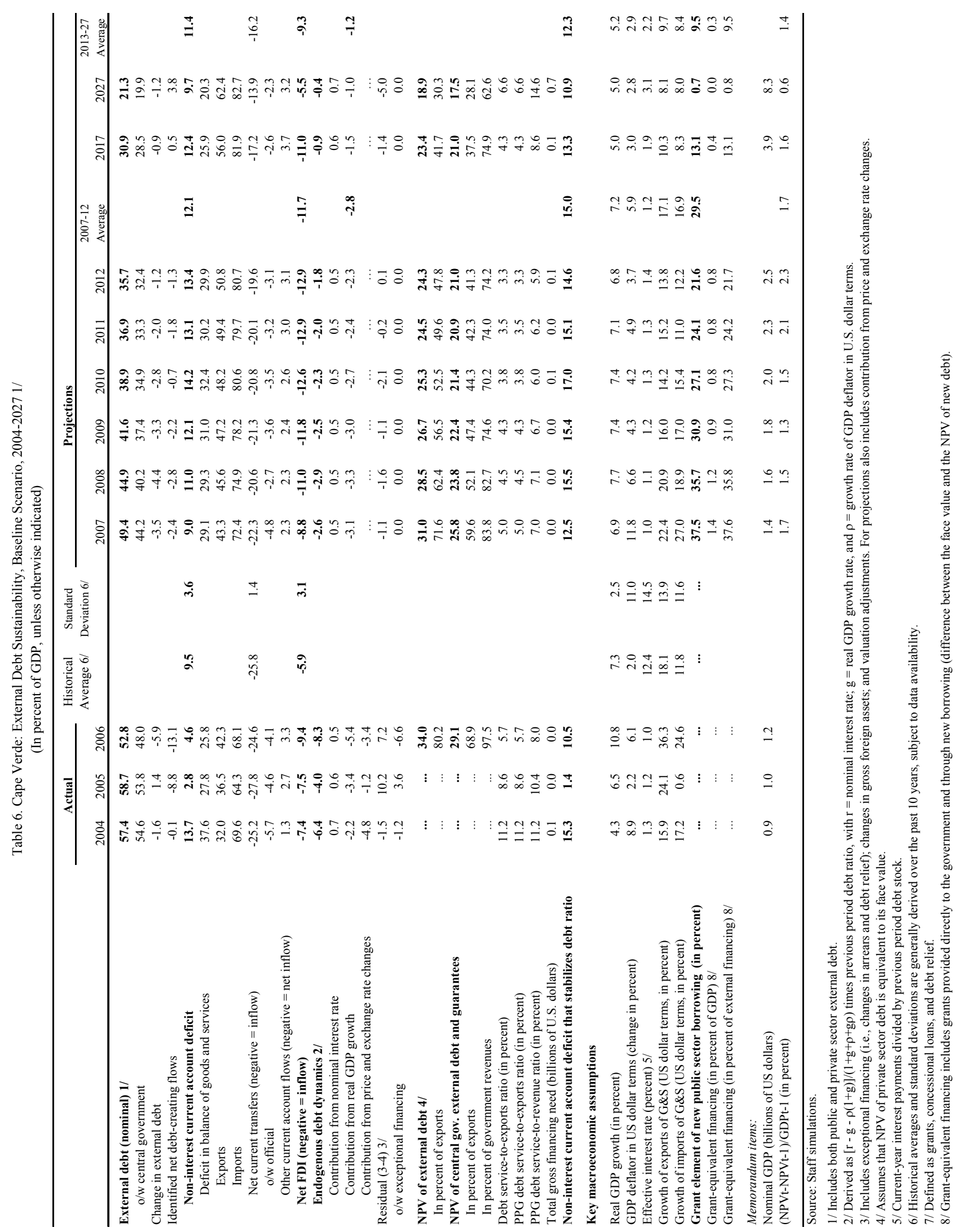


Table 7. Cape Verde: Sensitivity Analyses for Key Indicators of Central Government External Debt and Guarantees, 2007-27 (In percent)

\begin{tabular}{|c|c|c|c|c|c|c|c|c|}
\hline & \multicolumn{8}{|c|}{ Projections } \\
\hline & 2007 & 2008 & 2009 & 2010 & 2011 & 2012 & 2017 & 2027 \\
\hline Baseline & 26 & 24 & 22 & 21 & 21 & 21 & 21 & 18 \\
\hline \multicolumn{9}{|l|}{ A. Alternative Scenarios } \\
\hline A1. Key variables at their historical averages in $2008-27$ 1/ & 26 & 28 & 29 & 28 & 30 & 30 & 28 & 16 \\
\hline A2. New public sector loans on less favorable terms in $2008-27$ 2/ & 26 & 25 & 24 & 23 & 23 & 24 & 26 & 26 \\
\hline Scaling-up scenario & 23 & 24 & 24 & 24 & 25 & 25 & 23 & 18 \\
\hline \multicolumn{9}{|l|}{ B. Bound Tests } \\
\hline B1. Real GDP growth at historical average minus one standard deviation in 2008-09 & 26 & 24 & 24 & 23 & 22 & 22 & 22 & 18 \\
\hline B2. Export value growth at historical average minus one standard deviation in 2008-09 3/ & 26 & 30 & 38 & 32 & 28 & 24 & 21 & 18 \\
\hline B4. Net non-debt creating flows at historical average minus one standard deviation in 2008-09 & 26 & 28 & 32 & 28 & 25 & 23 & 21 & 18 \\
\hline B5. Combination of B1-B4 using one-half standard deviation shocks & 26 & 29 & 37 & 33 & 30 & 28 & 26 & 22 \\
\hline B6. One-time 30 percent nominal depreciation relative to the baseline in 20085 / & 26 & 33 & 31 & 30 & 29 & 29 & 29 & 24 \\
\hline
\end{tabular}

\section{NPV of debt-to-exports ratio}

Baseline

\section{A. Alternative Scenarios}

A1. Key variables at their historical averages in $2007-261 /$

A2. New public sector loans on less favorable terms in 2007-26 2/

Scaling-up scenario
60

52
47

$\begin{array}{llll}60 & 61 & 62 & 59\end{array}$

$60-54-50-58-58$

\section{B. Bound Tests}

B1. Real GDP growth at historical average minus one standard deviation in 2008-09 B2. Export value growth at historical average minus one standard deviation in 2008-09 3/ B3. US dollar GDP deflator at historical average minus one standard deviation in 2008-09 B4. Net non-debt creating flows at historical average minus one standard deviation in 2008-09 B5. Combination of B1-B4 using one-half standard deviation shocks

B6. One-time 30 percent nominal depreciation relative to the baseline in 2008 5/

\section{NPV of debt-to-revenue ratio}

Baseline

\section{A. Alternative Scenarios}

A1. Key variables at their historical averages in 2007-26 1/ A2. New public sector loans on less favorable terms in 2007-26 2/ Scaling-up scenario

\section{B. Bound Tests}

B1. Real GDP growth at historical average minus one standard deviation in 2008-09 B2. Export value growth at historical average minus one standard deviation in 2008-09 3/ B3. US dollar GDP deflator at historical average minus one standard deviation in 2008-09 B4. Net non-debt creating flows at historical average minus one standard deviation in 2008-09 B5. Combination of B1-B4 using one-half standard deviation shocks B6. One-time 30 percent nominal depreciation relative to the baseline in 2008 5/ 
Table 7. Cape Verde: Sensitivity Analyses for Key Indicators of Central Government External Debt and Guarantees, 2007-27 (continued) (In percent)

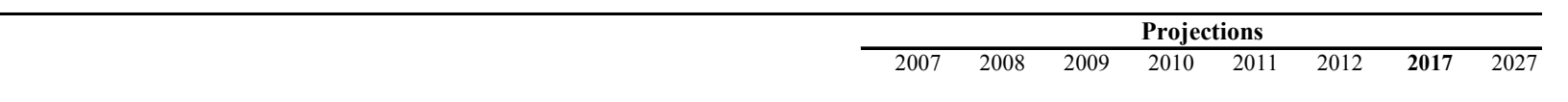

\section{Debt service-to-exports ratio}

Baseline

A. Alternative Scenarios

A1. Key variables at their historical averages in 2008-27 1/

A2. New public sector loans on less favorable terms in 2008-27 2/

Scaling-up scenario

\section{B. Bound Tests}

B1. Real GDP growth at historical average minus one standard deviation in 2008-09 B2. Export value growth at historical average minus one standard deviation in 2008-09 3/ B3. US dollar GDP deflator at historical average minus one standard deviation in 2008-09 B4. Net non-debt creating flows at historical average minus one standard deviation in 2008-09 B5. Combination of B1-B4 using one-half standard deviation shocks

B6. One-time 30 percent nominal depreciation relative to the baseline in 2008 5/

5

\begin{tabular}{|c|c|c|c|c|c|c|c|}
\hline 5 & 5 & 6 & 7 & 8 & 9 & 8 & 7 \\
\hline 5 & 5 & 4 & 4 & 3 & 3 & 4 & 11 \\
\hline 5 & 5 & 6 & 6 & 7 & 7 & 5 & 7 \\
\hline
\end{tabular}

\section{Debt service-to-revenue ratio}

Baseline

$\begin{array}{rrrrrrrr}5 & 5 & 4 & 4 & 4 & 3 & \mathbf{4} & 7 \\ 5 & 5 & 10 & 17 & 15 & 13 & \mathbf{6} & 8 \\ 5 & 5 & 4 & 4 & 4 & 3 & 4 & 7 \\ 5 & 5 & 7 & 9 & 8 & 8 & \mathbf{4} & 7 \\ 5 & 5 & 6 & 10 & 9 & 8 & \mathbf{5} & 7 \\ 5 & 5 & 4 & 4 & 4 & 3 & 4 & 7\end{array}$

\section{A. Alternative Scenarios}

A1. Key variables at their historical averages in 2008-27 1/

A2. New public sector loans on less favorable terms in 2008-27 2/

Scaling-up scenario

Debt service-to-revenue ratio

\section{B. Bound Tests}

B1. Real GDP growth at historical average minus one standard deviation in 2008-09

B2. Export value growth at historical average minus one standard deviation in 2008-09 3/

B3. US dollar GDP deflator at historical average minus one standard deviation in 2008-09

B4. Net non-debt creating flows at historical average minus one standard deviation in 2008-0 9

B5. Combination of B1-B4 using one-half standard deviation shocks

B6. One-time 30 percent nominal depreciation relative to the baseline in 2008 5/

7

7

Memorandum item:

Grant element assumed on residual financing (i.e., financing required above baseline) 6/

\begin{tabular}{|c|c|c|c|c|c|c|c|}
\hline 7 & 7 & 10 & 12 & 13 & 15 & 17 & 15 \\
\hline 7 & 7 & 6 & 6 & 6 & 5 & 9 & 24 \\
\hline 7 & 7 & 9 & 10 & 12 & 13 & 11 & 16 \\
\hline
\end{tabular}

Source: Staff projections and simulations.

1/ Variables include real GDP growth, growth of GDP deflator (in U.S. dollar terms), non-interest current account in percent of GDP, and non-debt creating flows.

2 / Assumes that the interest rate on new borrowing is by 2 percentage points higher than in the baseline., while grace and maturity periods are the same as in the baseline.

3/ Exports values are assumed to remain permanently at the lower level, but the current account as a share of GDP is assumed to return to its baseline level after the shoc] an offsetting adjustment in import levels).

4/ Includes official and private transfers and FDI.

5/ Depreciation is defined as percentage decline in dollar/local currency rate, such that it never exceeds 100 percent.

6/ Applies to all stress scenarios except for A2 (less favorable financing) in which the terms on all new financing are as specified in footnote 2. 
Figure 1. Cape Verde: Indicators of Central Government External Debt and Guarantees Under Alternative Scenarios, 2007-2027
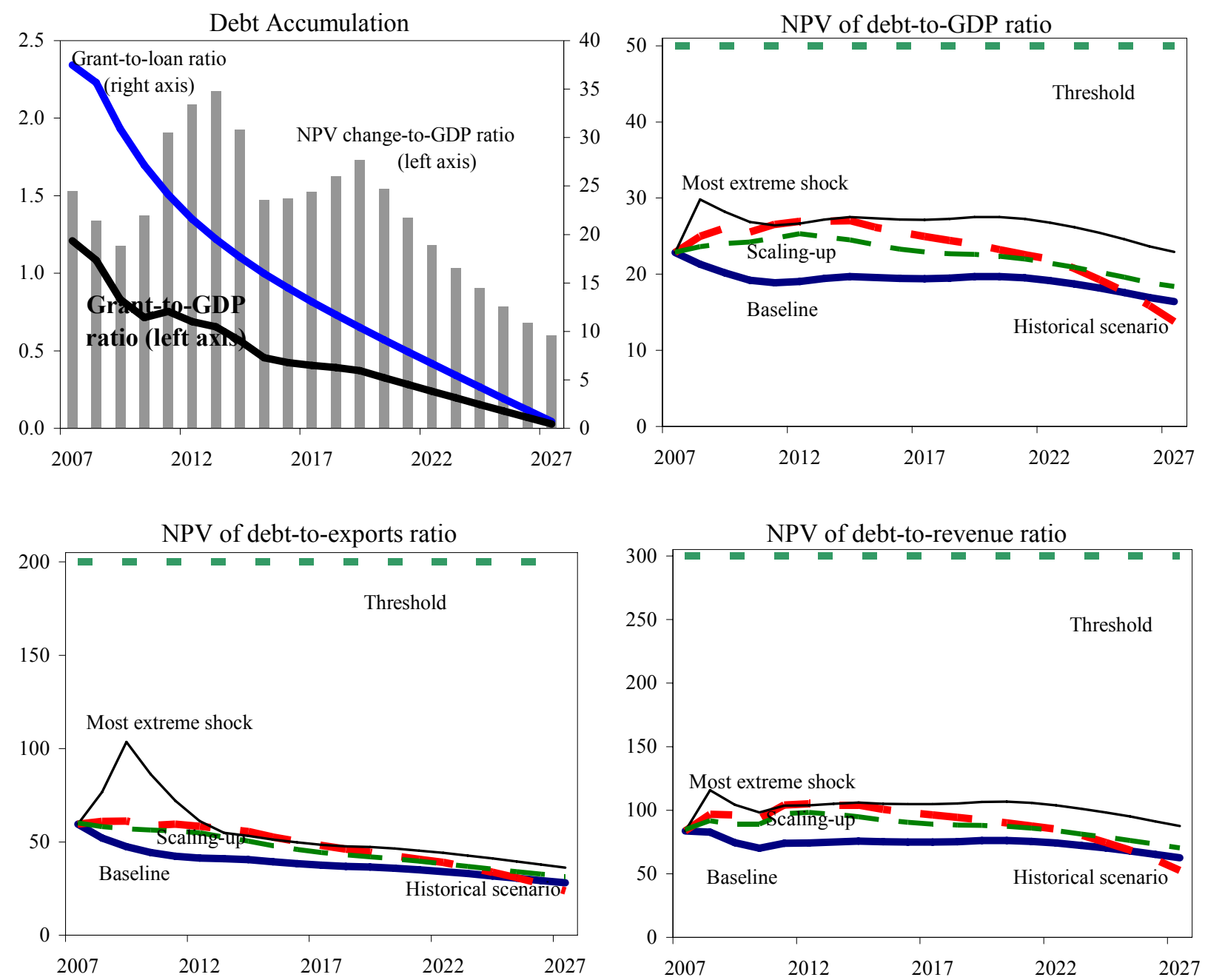

Debt-service-to-exports ratio
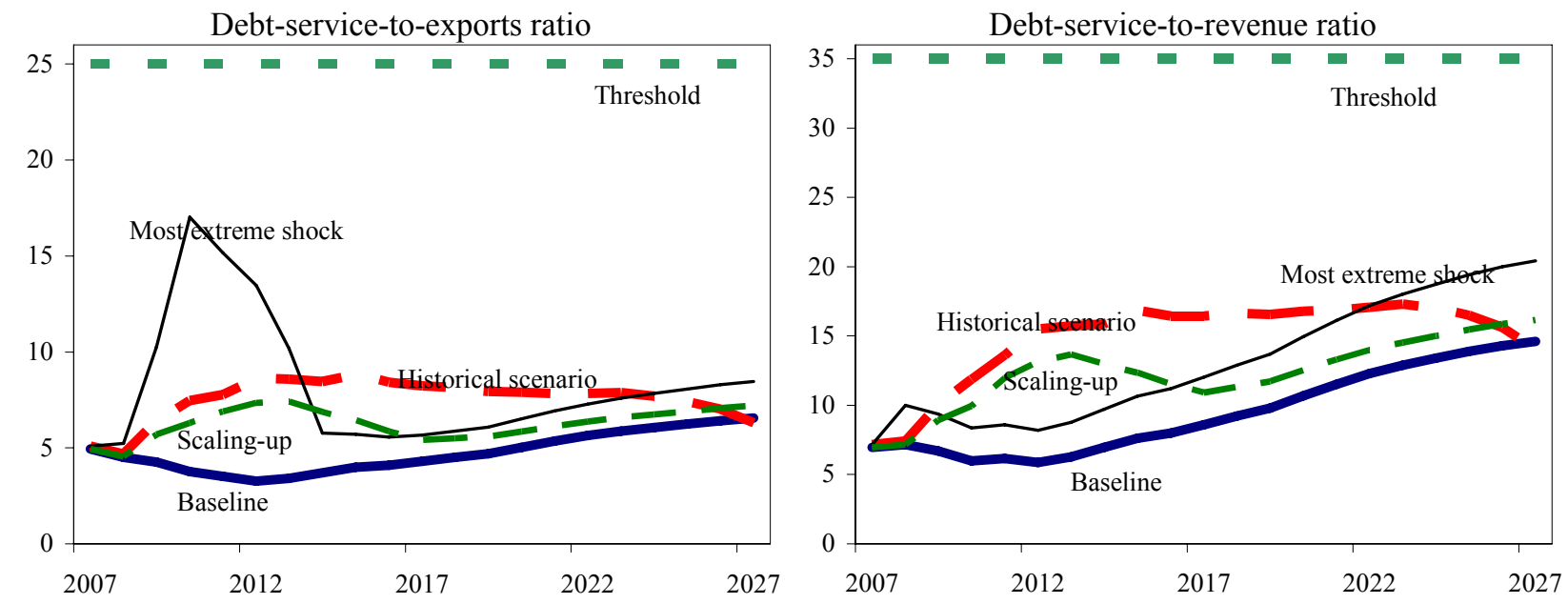

Source: Staff projections and simulations. 


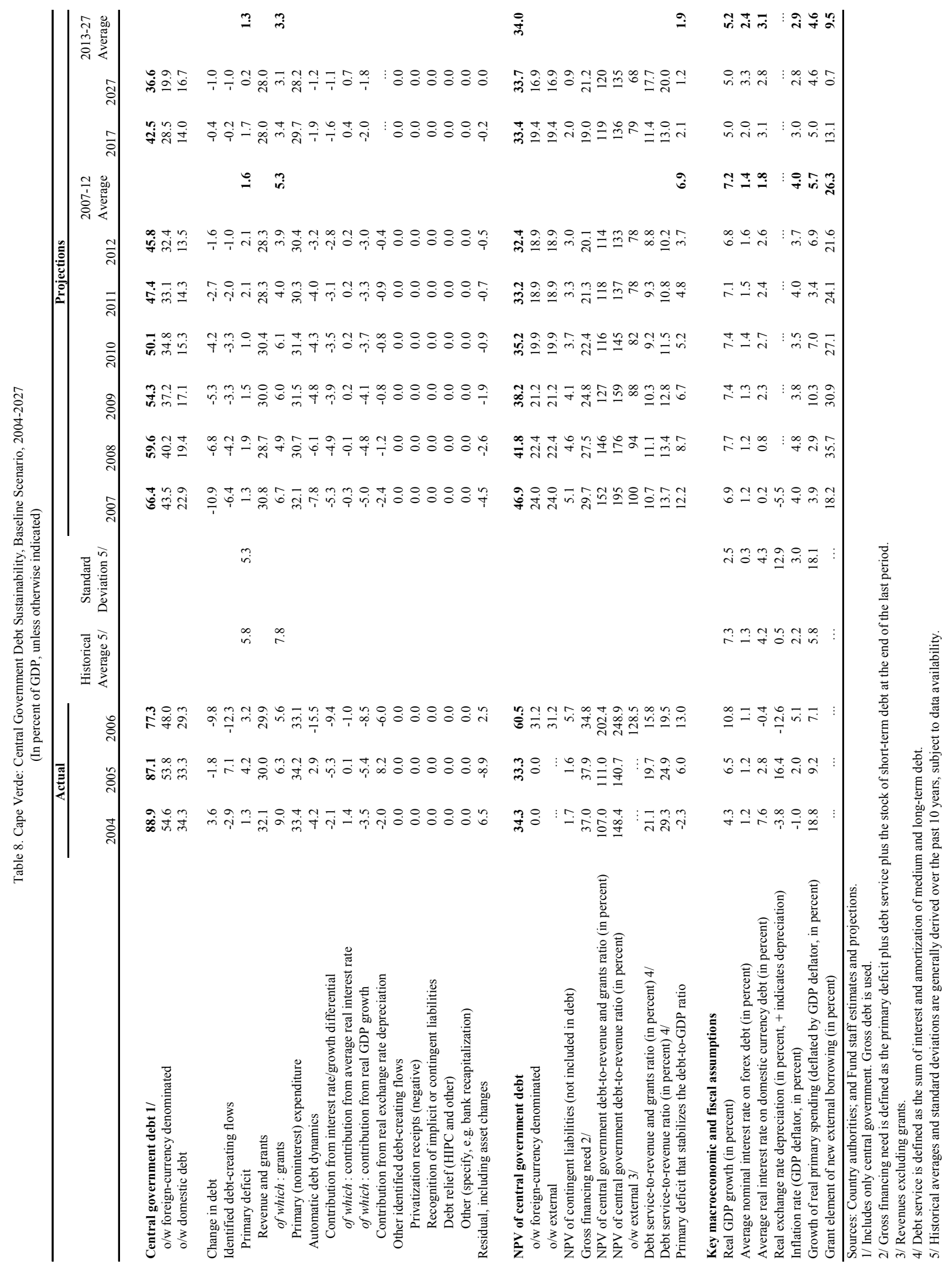


Table 9. Cape Verde: Sensitivity Analysis for Key Indicators of Central Government Debt, 2007-2027

\begin{tabular}{|c|c|c|c|c|c|c|c|c|}
\hline & \multicolumn{8}{|c|}{ Projections } \\
\hline & 2007 & 2008 & 2009 & 2010 & 2011 & 2012 & 2017 & 2027 \\
\hline \multicolumn{9}{|c|}{ NPV of Debt-to-GDP Ratio } \\
\hline Baseline & 47 & 42 & 38 & 35 & 33 & 32 & 33 & 34 \\
\hline \multicolumn{9}{|l|}{ A. Alternative scenarios } \\
\hline A1. Real GDP growth and primary balance are at historical averages & 47 & 46 & 46 & 48 & 49 & 51 & 64 & 89 \\
\hline A2. Primary balance is unchanged from 2007 & 47 & 41 & 38 & 35 & 32 & 31 & 29 & 33 \\
\hline A3. Permanently lower GDP growth $1 /$ & 47 & 42 & 39 & 37 & 35 & 35 & 42 & 63 \\
\hline Scaling-up scenario & 47 & 42 & 40 & 39 & 39 & 39 & 41 & 40 \\
\hline \multicolumn{9}{|l|}{ B. Bound tests } \\
\hline B1. Real GDP growth is at historical average minus one standard deviatio & 47 & 44 & 42 & 40 & 39 & 40 & 46 & 57 \\
\hline B2. Primary balance is at historical average minus one standard deviations & 47 & 51 & 57 & 53 & 50 & 49 & 48 & 45 \\
\hline B3. Combination of B1-B2 using one half standard deviation shocks & 47 & 49 & 53 & 49 & 46 & 44 & 43 & 41 \\
\hline B4. One-time 30 percent real depreciation in 2008 & 47 & 51 & 46 & 43 & 40 & 38 & 38 & 40 \\
\hline B5. 10 percent of GDP increase in other debt-creating flows in 2008 & 47 & 52 & 48 & 44 & 42 & 41 & 41 & 40 \\
\hline
\end{tabular}

\section{NPV of Debt-to-Revenue Ratio 2/}

Baseline

\section{A. Alternative scenarios}

A1. Real GDP growth and primary balance are at historical averages

A2. Primary balance is unchanged from 2007

A3. Permanently lower GDP growth $1 /$

Scaling-up scenario

\section{B. Bound tests}

B1. Real GDP growth is at historical average minus one standard deviatio B2. Primary balance is at historical average minus one standard deviation

B3. Combination of B1-B2 using one half standard deviation shocks

B4. One-time 30 percent real depreciation in 2008

B5. 10 percent of GDP increase in other debt-creating flows in 2008

152

146

127

116

118

114

$119 \quad 120$

$\begin{array}{llllllll}152 & 159 & 154 & 157 & 173 & 180 & \mathbf{2 3 1} & 326 \\ 152 & 143 & 125 & 115 & 114 & 108 & \mathbf{1 0 4} & 120 \\ 152 & 147 & 130 & 120 & 124 & 124 & \mathbf{1 4 9} & 224 \\ 152 & 145 & 134 & 129 & 137 & 140 & \mathbf{1 4 6} & 143\end{array}$

\section{Debt Service-to-Revenue Ratio 2/}

Baseline

11

$\begin{array}{llllllll}152 & 151 & 139 & 131 & 138 & 139 & \mathbf{1 6 5} & 202 \\ 152 & 177 & 188 & 173 & 177 & 172 & \mathbf{1 7 0} & 162 \\ 152 & 170 & 174 & 159 & 162 & 157 & \mathbf{1 5 5} & 146 \\ 152 & 177 & 155 & 140 & 140 & 135 & \mathbf{1 3 7} & 141 \\ 152 & 180 & 159 & 146 & 149 & 145 & \mathbf{1 4 6} & 142\end{array}$

\section{A. Alternative scenarios}

A1. Real GDP growth and primary balance are at historical averages

A2. Primary balance is unchanged from 2007

A3. Permanently lower GDP growth 1/

Scaling-up scenario

$\begin{array}{rrrrrrrr}11 & 11 & 17 & 23 & 32 & 37 & \mathbf{6 6} & 126 \\ 11 & 11 & 9 & 8 & 9 & 7 & \mathbf{4} & 15 \\ 11 & 11 & 11 & 10 & 11 & 11 & \mathbf{2 2} & 63 \\ 11 & 11 & 12 & 13 & 15 & 16 & \mathbf{1 4} & 20\end{array}$

\section{B. Bound tests}

B1. Real GDP growth is at historical average minus one standard deviatio B2. Primary balance is at historical average minus one standard deviations B3. Combination of B1-B2 using one half standard deviation shocks B4. One-time 30 percent real depreciation in 2008

B5. 10 percent of GDP increase in other debt-creating flows in 2008

\begin{tabular}{llllllll}
11 & 11 & 12 & 13 & 15 & 17 & $\mathbf{3 0}$ & 56 \\
11 & 11 & 26 & 40 & 41 & 39 & $\mathbf{3 8}$ & 40 \\
11 & 11 & 22 & 31 & 32 & 30 & $\mathbf{2 9}$ & 30 \\
11 & 11 & 11 & 10 & 11 & 11 & $\mathbf{1 5}$ & 26 \\
11 & 11 & 27 & 25 & 26 & 25 & $\mathbf{2 6}$ & 29 \\
\hline
\end{tabular}

Sources: Country authorities; and Fund staff estimates and projections.

$1 /$ Assumes that real GDP growth is at baseline minus one standard deviation divided by the square root of 20 (i.e., the length of the projectio 2/ Revenues are defined inclusive of grants. 
Figure 2. Cape Verde: Indicators of Public Debt Under Alternative Scenarios, 2007-2027 1/

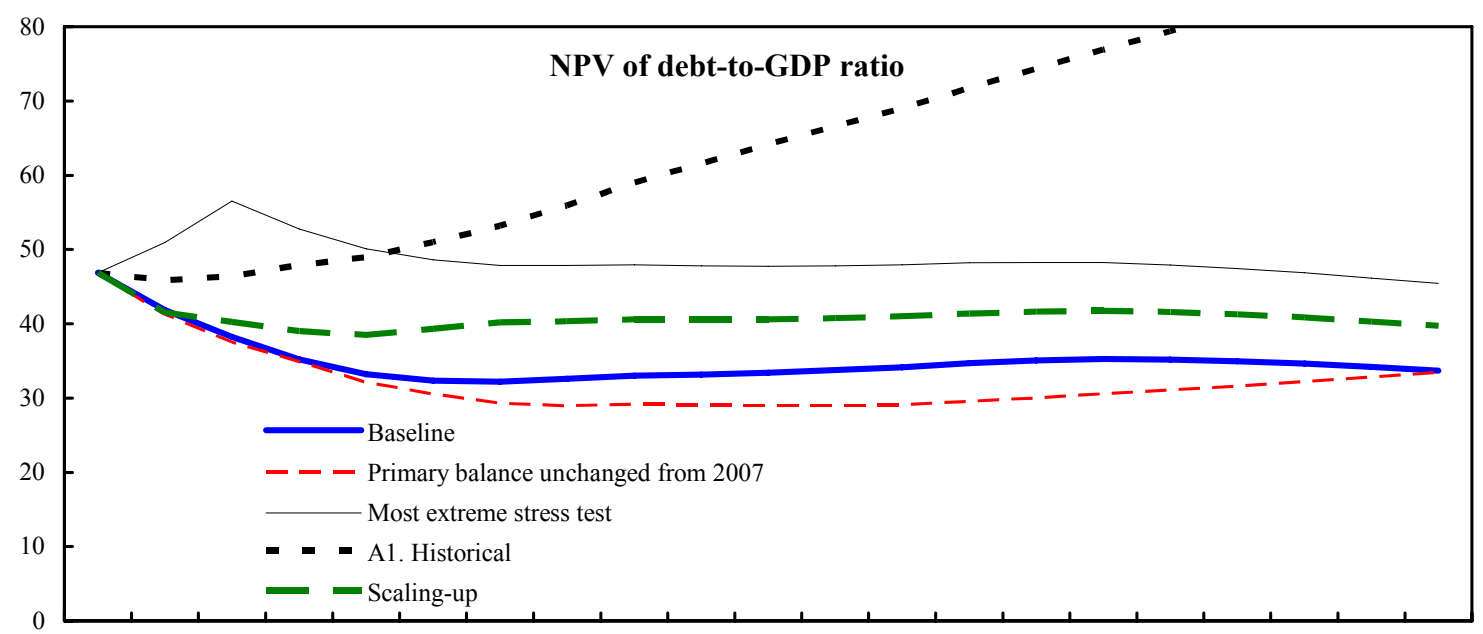

200720082009201020112012201320142015201620172018201920202021202220232024202520262027
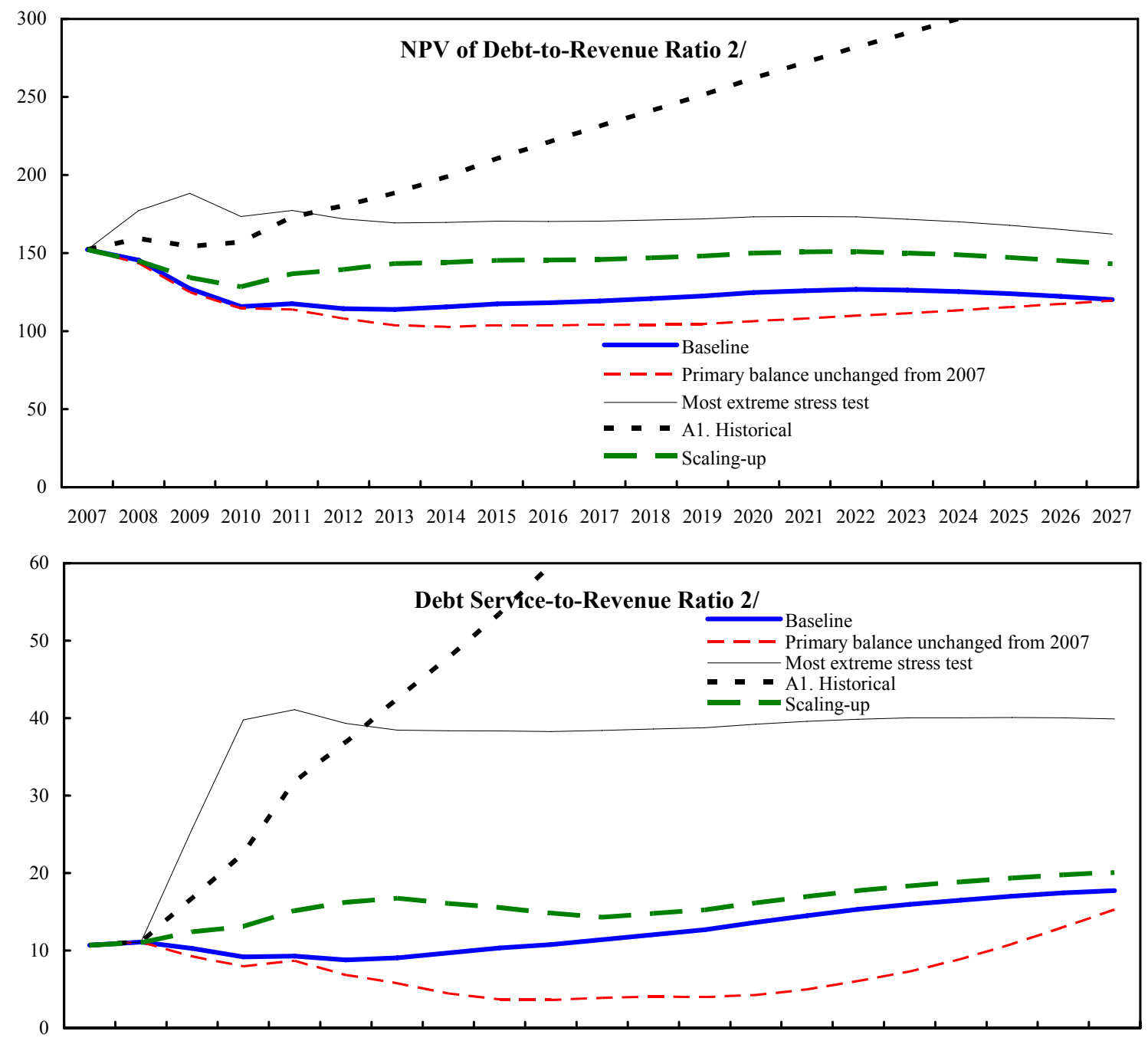

200720082009201020112012201320142015201620172018201920202021202220232024202520262027

Source: Staff projections and simulations.

1/ Most extreme stress test is test that yields highest ratio in 2017.

2/ Revenue including grants. 
Press Release No. 07/307

FOR IMMEDIATE RELEASE

December 21, 2007

International Monetary Fund

Washington, D.C. 20431 USA

\section{IMF Executive Board Completes Third Review Under the Policy Support Instrument for Cape Verde}

The Executive Board of the International Monetary Fund (IMF) today completed the third review under a three-year Policy Support Instrument (PSI) for Cape Verde. The PSI was approved on July 31, 2006 (see Press Release No. 06/172).

Cape Verde's PSI is designed to enhance the sustainability of growth and development by maintaining a stable macroeconomic environment and moving forward with structural reforms. It is also expected to help the country reduce macroeconomic risks, provide a margin for safety against shocks, and prepare for a possible longer-term decline in access to concessional external financing. Key measures are directed to reducing public debt, building up international reserves, improving public financial management, and strengthening financial sector and energy sector regulation.

The IMF's framework for PSIs is designed for low-income countries (and small island states) that may not need, or want, IMF financial assistance, but still seek IMF advice, monitoring and endorsement of their policies. PSIs are voluntary and demand driven. PSIsupported programs are based on country-owned poverty reduction strategies adopted in a participatory process involving civil society and development partners and articulated in a Poverty Reduction Strategy Paper (PRSP). This is intended to ensure that PSI-supported programs are consistent with a comprehensive framework for macroeconomic, structural and social policies to foster growth and reduce poverty. Members' performance under a PSI is normally reviewed semi-annually, irrespective of the status of the program (see Public Information Notice No. 05/145).

Following the Executive Board's discussion of Cape Verde, Mr. Murilo Portugal, Deputy Managing Director and acting Chair, stated:

“The Cape Verde authorities' well-crafted program under the PSI is guided by the need to support macroeconomic stability and the exchange rate peg, reduce domestic debt, increase foreign reserves, and create fiscal space to accommodate the expected decline in concessional external financing as the country graduates from LDC status. 
"Cape Verde continues to demonstrate a strong economic performance, and the authorities are to be commended for their prudent macroeconomic management in recent years. Growth is being sustained, bolstered by significant increases in foreign direct investment, especially in the tourism sector. Reflecting this, unemployment and poverty rates are falling. Although inflation has increased in recent months, this largely reflects poor rainfall, which temporarily drove up food prices. Underlying inflation pressures remain moderate.

"Fiscal consolidation and the build-up of official reserves have proceeded faster than program expectations. The targets for both domestic debt and official reserves initially set for 2009 are likely to be reached in 2008. While the authorities have made progress in preventing an accumulation of central government arrears, challenges remain in the areas of financial sector regulation and supervision, public financial management, and energy sector reform.

"It is important to strengthen financial sector regulation and supervision. In that regard, the authorities' plans to establish a financial intelligence unit and strengthen the framework to combat money-laundering and the financing of terrorism in 2008 are welcome.

"Progress is being made in strengthening public sector financial management. Weaknesses in data reporting understated the deviation with respect to the extent of net domestic borrowing. Since the excess borrowing had little effect on the economic outturn, and has now been unwound, and as measures have been taken to improve budget execution and monitoring, the Board decided to maintain a positive assessment of Cape Verde's past program performance under the PSI.

"Strengthening the energy sector will be critical for enhancing growth and poverty reduction and limiting fiscal risk. In that regard, it is important that the authorities implement rapidly their plans for a comprehensive overhaul of the energy sector, including establishing a new base utility tariff structure and continuously applying the fuel and utility price adjustment mechanisms. This would depoliticize price setting, safeguard the budget, and give companies in the energy sector incentives for investment and efficiency gains," Mr. Portugal said. 


\section{Statement by Laurean W. Rutayisire, Executive Director for Cape Verde}

\section{December 21, 2007}

1. Cape Verde continues to maintain a productive program relationship with the Fund in the context of the PSI. On behalf of the country's authorities, I would like to thank the Board, Management, and the staff for playing a useful role in facilitating this positive outcome. I also appreciate the constructive and candid dialogue that staff held with the authorities during the last review mission to Cape Verde. As usual, my authorities have expressed their consent to the Fund publication of their letter of intent and the staff report.

2. The authorities are aware that the imminent graduation of Cape Verde from the UN least-developed-country status, while appreciable, may put a burden on the country's future ability to draw concessional resources. However, they view this development as an opportunity to strengthen revenue mobilization, promote private sector development, and attract capital flows, so as to more than offset any potential decline in concessional assistance. They remain firmly attached to the objectives of the PSI, including the reduction in government debt, safeguard of the exchange rate regime, and the strengthening of public sector management and the financial sector. As discussed below, these objectives are being achieved according to program schedule and some are even surpassed as a result of sound macroeconomic policies implemented by the authorities. Yet, the authorities are determined to continue to press forward with their reform agenda and in this endeavor they would welcome continued Fund advice and technical assistance.

\section{Economic And Policy Performance Under the PSI}

3. Supported by the authorities' strong policies, Cape Verde's economic performance under the PSI continues to be impressive. Latest available data point to double-digit level of growth nearing 11 percent in 2006, mainly driven by the telecommunications and tourism sectors and construction. Concomitantly, the rate of unemployment is estimated to have decreased significantly. In anticipation of the continuous dynamism of these sectors, growth is also projected to remain strong at about 7 percent in 2007. After its spike in late-2006, consumer inflation has since continued to decelerate and it is expected to be subdued in 2008.

4. Macroeconomic performance under the PSI continues to be satisfactory, reflecting the authorities' good record of sound economic management. With the conduct of strong and prudent macroeconomic policies, all quantitative assessment criteria were met at end-June 2007 with comfortable margins and initial program objectives were surpassed in a number of 
areas. Thus, domestic debt reduction proceeded more rapidly than projected at the beginning of the PSI arrangement. At the same time reserve accumulation continued to be made at a faster pace than initially targeted under the PSI.

5. On the structural front, the authorities' efforts to improve tax administration contributed to the mobilization of higher-than-projected tax revenues. Against this backdrop, implementation of the structural reforms was affected by nonobservance of two assessment criteria on the full implementation of mechanisms for setting base utility tariffs and adjusting electricity, water, and fuel prices. Key corrective measures were subsequently taken by the authorities, which contributed to mitigating fiscal risks that emanate from the energy sector. In particular, the authorities proceeded in October to increases of 15 and 11 percent in the prices of retail gasoline and diesel respectively. During the same month, they presented to the National Assembly the 2008 budget which makes no room for energy and utility subsidies. In addition, they gave firm instructions to the economic regulatory agency (ARE) to make adjustments in fuel prices, as is necessary, so as to avoid building fiscal liabilities.

6. Going forward, a number of other measures included in the authorities' reform agenda will be critical to reduce potential risks associated with the energy sector. In particular, the authorities' plan to finalize and publish during the first quarter of 2008 a mechanism for setting base electricity tariffs will serve this purpose; so will the decisions already taken to render the price-setting mechanism of energy products more transparent. Moreover, as part of the authorities' energy sector development strategy, important investments are planned to increase the capacity and improve the efficiency of the energy and water sectors. Materialization of these plans which is expected to entail increased private sector involvement in the energy sector's management and investment decisions will also play an important role in reducing fiscal liabilities inherent to the sector.

7. In light of the above I would appreciate Directors' support for the granting of waivers for the two missed assessment criteria.

\section{POLICY AND REForm AgENDA}

8. Cape Verde's growth outlook continues to be favorable. In 2008, real GDP is forecasted to grow by about $73 / 4$ percent given the anticipated strong performance in the tourism sector and infrastructure investment. In the pursuit of sustained and strong private sector-led growth, the authorities intend to consolidate macroeconomic stability, improve public sector financial management and tax administration, and strengthen regulation and supervision of the financial sector while taking the abovementioned steps to reduce fiscal risks. 


\section{Consolidating Macroeconomic Stability}

9. The authorities' commitment to maintain prudent macroeconomic policies should facilitate materialization of the favorable growth prospects and help safeguard macroeconomic stability. The 2008 budget builds on conservative estimates of the projected evolution of expenditures and revenues, which is in line with the authorities' strong record of fiscal discipline. With the authorities' decision to avoid additional net borrowing, further domestic debt reduction is expected in 2008. This should help meet the targeted 20 percent debt-to-GDP ratio with a comfortable margin by the end of the program period. In parallel, the authorities are taking a number of steps to avoid accumulation of arrears both at the levels of the government and the municipalities. These include the efforts made to assist government entities in their efforts to improve budget execution and payment practices and the decision to clear any arrears accumulated by these entities and deduct corresponding amounts from government transfers made in their favor.

10. In support of the peg to the euro and in line with PSI objectives, monetary policy will continue to be geared at further accumulating foreign exchange reserves. Efforts by the monetary authorities to build reserves will be accompanied by active steps to manage liquidity through appropriate issuance of short-term bills by the Central Bank of Cape Verde $(\mathrm{BCV})$. At the same time, the $\mathrm{BCV}$ will continue to closely monitor interest rate differentials with the euro area, notably with a view to avoiding unsuitable shifts in remittance inflows.

\section{Improving public sector financial management and tax administration}

11. Cape Verde's public sector financial management (PFM) system compares favorably with that of other countries with similar circumstances. The country's Integrated Online Budget Management System (SIGOF) is supported by a technological platform that allows for real-time availability of some data on budget execution, thus facilitating budgetary consolidation and the monitoring and evaluation of budget management. Nevertheless, the authorities concur that there is still scope for PFM reforms to further strengthen budget management. In this respect, they are taking steps to expand SIGOF to most municipalities and semi-autonomous institutes and to decentralize budget execution. A new Chart of Public Accounts building on modern accounting principles has been approved and it is scheduled to be implemented in 2008. Legislation on a new budget framework was submitted to the National Assembly. The authorities agreed to conduct systematically a formal mid-year review of fiscal developments with a view to taking any corrective actions that may be needed. They have decided to set November 30 as the deadline for making any new spending commitments in order to ensure better control over such commitments. In order to help the authorities press forward with their PFM reforms, Fund technical assistance would be valuable. 
12. The authorities will pursue their ongoing efforts to improve tax administration. Building notably on the recommendations of recent IMF technical assistance, they are taking steps to enhance tax collection, especially among large taxpayers. They have prepared for submission to the National Assembly a series of documents with the aim at strengthening the tax framework, including a draft General Tax Code, a new Code on Judicial Processes, and draft personal and corporal income tax bills. In parallel, the authorities are working to streamline tax exemptions and incentives and, in this process, they have expressed their continued interest in IMF technical assistance. Progress is also being made toward strengthening audit processes. In this regard, a customs audit court was established and a draft legislation relating to the Court of Auditors was submitted to the National Assembly. In addition, more frequent recourse to tax audits is expected going forward given the recruitment of additional tax inspectors.

\section{Strengthening financial sector regulation and supervision}

13. The Bank of Cape Verde (BCV) continues to take steps to strengthen its framework for regulation and supervision of the financial sector. It envisages to undertake in 2008 some measures aimed at improving compliance with international standards on banking accounting. Progress is also being made in strengthening regulations on banks' capital ratios and procedures for bank licensing. The authorities would greatly appreciate an FSAP for Cape Verde in support of their continuous efforts to promote financial sector development and to address potential vulnerabilities facing the sector.

14. The BCV is contemplating further liberalization of the capital account which would basically corroborate the circumstances under which financial flows are currently being operated in Cape Verde. The BCV is also in the process of reaching information sharing agreements with supervisory bodies in the home country of most institutions currently active in the offshore financial center. Moreover, it is the authorities' intention to submit to the National Assembly bills aimed at strengthening the framework for fighting money laundering and combating the financing of terrorism. Consistent with the AML/CFT recommendations made by a Fund assessment mission, these will include draft AML/CFT legislation and proposal for the establishment of a financial intelligence unit.

\section{MISREPORTING ISSUE}

15. At the time of the second review of the PSI, the authorities requested, and the Board granted, a waiver for the nonobservance of the assessment criterion on net domestic borrowing, as the program ceiling on domestic borrowing was reported to be exceeded by 0.8 percent of GDP. It later appeared to the authorities that excess net domestic borrowing relative to program ceiling was 1.3 percentage points of GDP higher than initially reported, and that the underreporting was due to computing errors made in pulling data off SIGOF. We share staff's conclusion that this misreporting does not change the favorable assessment of 
Cape Verde's performance under the PSI. That being the case, Cape Verde's experience would illustrate the fact that the magnitude of the deviation from the assessment (or performance) criterion is not necessarily critical to the assessment of program performance. ${ }^{20}$ This implies that a misreporting may be significant in terms of its size but clearly insignificant in terms of its consequences on the assessment of program performance. And in our view it would be appropriate that any such misreporting be considered as "de minimis".

16. We thus believe that the Cape Verde's case appears to provide a new opportunity for further refinement of the guidelines on misreporting. Indeed, there seems to be merit in initiating the necessary steps to revisit the misreporting policies, so as to consider "de minimis" misreporting cases only those which have an impact on the assessment of program performance. Such steps would be useful in helping avoid the undesirable situation where a decision to consider a misreporting not "de minimis" is based exclusively on the size of the deviation from the assessment or performance criterion. This would be especially helpful in the current circumstances where the meaning of a deviation "so small as to be trivial" is widely open to interpretation. ${ }^{21}$ We would hope that the Board will take, in due course, the necessary steps in this direction.

\section{CONCLUSION}

17. Cape Verde continues to make notable progress toward the achievement of the PSI objectives, reflecting the credibility of the authorities' reform agenda. Debt reduction continues to proceed satisfactorily with net domestic debt in percent of GDP following a steep downward trend. The pace of reserve accumulation exceeds initial expectations. Notable improvements in public sector financial management are being achieved, as reflected by the significant progress made in improving arrears prevention and public accounting. A number of steps taken by the authorities are expected to help further strengthen financial sector supervision and regulation. Reforms implemented in the energy sector are helping reduce fiscal risks associated with the sector.

18. In light of the above, I call on Directors to consider favorably the authorities' request for waiver for the two missed assessment criteria as well as the misreporting occasioned by accounting errors, and to support completion of the third review of the PSI for Cape Verde.

\footnotetext{
${ }^{20}$ In the context of a PSI existing misreporting policies suggest that "to be considered de minimis, a deviation from an assessment criterion or other specified condition should be so small as to be trivial with no impact on the assessment of performance under the member's program".

${ }^{21}$ Although examples of "de minimis" misreporting are reported in Table 1 of EBS/06/86, these do not specify any threshold under which a deviation from an assessment criterion can be considered small.
} 